Universidade de São Paulo

Instituto de Astronomia, Geofísica e Ciências Atmosféricas

Departamento de Astronomia

Daniel Moser Faes

\title{
Modelagem de nebulosas planetárias com morfologia bipolar
}

São Paulo 

Daniel Moser Faes

\section{Modelagem de nebulosas planetárias com morfologia bipolar}

Dissertação apresentada ao Departamento de Astronomia do Instituto de Astronomia, Geofísica e Ciências Atmosféricas da Universidade de São Paulo como requisito parcial para a obtenção do título de Mestre em Ciências.

Área de Concentração: Astronomia

Orientador: Prof. Dr. Roberto Dell'Aglio

Dias da Costa

São Paulo 
Nota: A versão original desta dissertação encontra-se disponível no Serviço de Biblioteca e Documentação do Instituto de Astronomia, Geofísica e Ciências Atmosféricas IAG/USP. 
Aos meus pais. 



\section{Agradecimentos}

Aos meus pais e queridos membros da minha família;

Ao orientador Roberto D.D. Costa, sem o qual a realização do trabalho não seria possível;

Aos amigos Alex C. Carciofi, Eduardo A. Guimarães e Odair M. Gimenes, pelo incentivo em momentos cruciais;

Aos colegas Christophe Morisset e Oscar Cavichia, pelas contribuições em ciência;

Aos leitores desta obra;

À CAPES, pelo apoio financeiro parcial;

À FAPESP, pelo apoio financeiro, sob o projeto $\mathrm{n}^{o}$ : 2009/01346-2;

À sociedade brasileira, por construir instituições como o IAG/USP e o LNA/MCT.

Esta tese/dissertação foi escrita em LATEX com a classe IAGTESE, para teses e dissertações do IAG. 



\section{Resumo}

A evolução de estrelas de massas próximas a do Sol culmina no objeto astrofísico denominado por "nebulosa planetária" (NP). Vários destes objetos podem ser resolvidos angularmente no céu, revelando diferentes morfologias e propriedades.

O presente trabalho propõe-se a mapear espacialmente as propriedades físicas de um conjunto destes objetos, de morfologia bipolar, e então efetuar sua modelagem. Para este mapeamento foram executadas observações do Observatório Pico dos Dias (LNA/MCT) através das técnicas observacionais de espectroscopia de fenda longa e de campo integral. As modelagens foram executadas com o código de fotoionização CLOUDY em seu formato pseudo tridimensional, CLOUDY_3D.

Nossos resultados mostram que as simulações são capazes de reproduzir em boa medida o quadro de observações realizadas, mesmo partindo de considerações simplificadoras para a construção do ambiente astrofísico analisado. Como produto, estabelece-se limites para os parâmetros dos objetos, tais como características da distribuição de matéria, propriedades da fonte central, distância e dimensões envolvidas.

Dos seis objetos com simulações apresentadas neste trabalho, quatro indicam ser NPs limitadas por densidade. Sub-estruturas puderam ser identificadas como desvios na simetria dos fluxos emergentes. A variação do valor de avermelhamento associado à razão de fluxos $\mathrm{H} \alpha / \mathrm{H} \beta$, observado ao longo da estrutura angular dos objetos, indica a ocorrência de ex-

tinção diferencial da nuvem. A avaliação de distintas regiões em diagramas de diagnóstico de plasma evidencia as características reproduzidas pelos modelos, e consequentemente a acurácia destes em relação aos dados observacionais. 



\section{Abstract}

The evolution of low and intermediate mass stars culminates in astrophysical objects named by planetary nebula $(\mathrm{PN})$. Several of these objects can be angularly resolved in the sky, revealing different morphologies and properties.

This study aims to map spatially the physical properties of a set of objects, with bipolar morphology, and then build their models. For this mapping, observations were performed at the Pico dos Dias Observatory (LNA / MCT) using the observational techniques of long slit and integral field spectroscopy. Modeling was performed with the photoionization code CLOUDY in its pseudo three-dimensional format, CLOUDY_3D.

Our results show that the simulations are able to reproduce the major features of observations, even taking simplifying assumptions for the astrophysical environment analyzed. As a product, it establishes limits for the parameters of objects, such as characteristics for the distribution of matter, properties of the central star, distances and related dimensions.

For six objects analyzed, four indicate that are PNe limited by density. Sub-structures could be identified as deviations in the symmetry of the emerging fluxes. The change in the reddening value associated to the ratio of line fluxes $\mathrm{H} \alpha / \mathrm{H} \beta$, observed along the angular structure of objects, indicates the occurrence of differential extinction in the nebulae. The evaluation of different regions in plasma diagnostic diagrams shows the features reproduced by the models, and consequently their accuracy in relation to observational data. 



\section{Lista de Figuras}

1.1 Morfologias assumidas por Nebulosas Planetárias. . . . . . . . . . . . . . . 22

2.1 Diagramas de energia das transições proibidas de OII e SII. . . . . . . . . . 35

2.2 Diagramas de energia das transições proibidas de OIII e NII. . . . . . . . . 37

4.1 Imageamento e posição da fenda para IC4406. . . . . . . . . . . . . . . . 50

4.2 Comparação entre observações e modelo de IC4406. . . . . . . . . . . . . . 51

4.3 Avermelhamento E(B-V) para IC4406. . . . . . . . . . . . . . . . 52

4.4 Imageamento e posição das fendas para NGC5844. . . . . . . . . . . . . . . 55

4.5 Avermelhamento E(B-V) para NGC5844. . . . . . . . . . . . . . . 55

4.6 Comparação entre observações e modelo de NGC5844, fenda central. . . . . 60

4.7 Comparação entre observações e modelo de NGC5844, fenda superior. . . . 61

4.8 Comparação entre observações e modelo de NGC5844, fenda inferior. . . 62

4.9 Imageamento de NGC6572. Coloração e orientação desconhecidos. . . . . . 63

4.10 Mapas de melhor ajuste em outros modelos considerados de NGC6572. 66

4.11 Comparação entre observação e modelo de NGC6572. . . . . . . . . . . . 68

4.12 Imageamento de IC2553. Intensidade $\log ([\mathrm{NII}]+\mathrm{H} \alpha)$. Orientação arbitrária. 69

4.13 Diagrama posição-velocidade de IC2553. . . . . . . . . . . . . . . . . . . . 69

4.14 Comparação entre observação e modelos de IC2553. . . . . . . . . . . . . . 73

4.15 Imageamento de NGC5882. . . . . . . . . . . . . . . . . . . . . 74

4.16 Comparação entre observação e modelo de NGC5882. . . . . . . . . . . . . 77

4.17 Imageamento de IC1297. . . . . . . . . . . . . . . . . . . . . 79

4.18 Comparação entre observação e modelo de IC1297. . . . . . . . . . . . . . 82

4.19 Imageamento de IC4776. . . . . . . . . . . . . . . . . . . . . . 83 
4.20 Comparação entre observação e modelos de IC4776 . . . . . . . . . . . . . 86

4.21 Diagramas de diagnóstico de plasma para IC4406 e NGC6572 . . . . . . . . 88

4.22 Diagramas de diagnóstico de plasma para IC4776 . . . . . . . . . . . . . 89 


\section{Lista de Tabelas}

1.1 Principais propriedades das Nebulosas Planetárias . . . . . . . . . . . . . 18

2.1 Objetos analisados com a técnica de espectroscopia de fenda longa . . . . . 28

2.2 Objetos analisados com a técnica de espectroscopia de campo integral . . . 29

2.3 Parâmetros da configuração observacional de fenda longa . . . . . . . . . . 29

2.4 Parâmetros da configuração observacional de campo integral ....... 30

4.1 Parâmetros físicos e composição química obtidos para IC4406. . . . . . . . 49

4.2 Comparação entre observações e modelo de IC4406. . . . . . . . . . . . . . 53

4.3 Parâmetros do modelo final de IC4406 comparados com referências. . . . . 54

4.4 Propriedades geométricas do modelo de IC4406. . . . . . . . . . . . . . 54

4.5 Parâmetros físicos e composição química obtidos para NGC5844 . . . . . . 56

4.6 Parâmetros obtidos para NGC5844 em outro posicionamento de fenda. . 57

4.7 Comparação entre observações e modelo de NGC5844. . . . . . . . . . . . 58

4.8 Parâmetros do modelo final de NGC5844 comparados com referências. . . . 59

4.9 Propriedades geométricas do modelo de NGC5844. . . . . . . . . . . . . 59

4.10 Valores de composição química de NGC6572 adotados para as simulações. . 63

4.11 Parâmetros do modelo final de NGC6572 comparados com referências. . . . 64

4.12 Propriedades geométricas do modelo de NGC6572 . . . . . . . . . . . . . . 65

4.13 Valores de $\chi^{2}$ entre o modelo final e as observações de NGC6572. . . . . . . 65

4.14 Valores de composição química de IC2553 adotados para as simulações. . 70

4.15 Parâmetros dos modelos finais de IC2553 comparados com referências. . . . 71

4.16 Propriedades geométricas do modelo LD (limitado por densidade) de IC2553. 72

4.17 Propriedades geométricas do modelo LI (limitado por ionização) de IC2553. 72 
4.18 Valores de $\chi^{2}$ entre os modelos finais e as observações de IC2553. . . . . . . 72

4.19 Parâmetros do modelo final de NGC5882 comparados com referências. . . . 75

4.20 Composição química utilizada para NGC5882 nas simulações. ..... . 76

4.21 Valores de $\chi^{2}$ entre o modelo final e as observações de NGC5882 . . . . . . 76

4.22 Propriedades geométricas do modelo de NGC5882 . . . . . . . . . . . . . 78

4.23 Propriedades geométricas do modelo de IC1297. . . . . . . . . . . . . . 80

4.24 Valores de composição química de IC1297 adotados para as simulações. 80

4.25 Parâmetros do modelo final de IC1297 comparados com referências. . . . . 81

4.26 Valores de $\chi^{2}$ entre o modelo final e as observações de NGC1297. . . . . . . 81

4.27 Composição química adotada para as simulações de IC4776. . . . . . . . . 83

4.28 Parâmetros dos modelos de IC4776, comparado com referências. . . . . . . 84

4.29 Valores de $\chi^{2}$ entre os modelos finais e as observações de IC4776. . . . . . . 84

4.30 Propriedades geométricas do modelo limitado por densidade (LD) de IC4776. 85

4.31 Propriedades geométricas do modelo limitado por ionização (LI) de IC4776. 85

A.1 Linhas cujos fluxos são procurados pela rotina pnflux. . . . . . . . . . . . 107

B.1 Lista dos objetos observados em missões ao OPD/LNA . . . . . . . . . . . 109

B.2 Observações do projeto com a técnica de espectroscopia de campo integral. 110

C.1 Fluxos observados por toda fenda e respectivas incertezas. . . . . . . . . . 111

C.2 Comparação entre fluxos de IC4406. Janelas 1 a $5 . \quad$. . . . . . . . . . . . . 112

C.3 Comparação entre fluxos de IC4406. Janelas 6 a 10. . . . . . . . . . . . . . 114

C.4 Comparação entre fluxos de IC4406. Janelas 11 a $13 . \quad$. . . . . . . . . . . . 115

C.5 Comparação entre fluxos de NGC5844. Janelas 1 a 5, fenda superior. . . . 116

C.6 Comparação entre fluxos de NGC5844. Janelas 6 a 8, fenda superior. . . . 118

C.7 Comparação entre fluxos de NGC5844. Janelas 1 a 5, fenda central. . . . . 119

C.8 Comparação entre fluxos de NGC5844. Janelas 6 a 10, fenda central. . . . 120

C.9 Comparação entre fluxos de NGC5844. Janelas 1 a 5, fenda inferior. . . . . 122

C.10 Comparação entre fluxos de NGC5844. Janelas 6 a 8, fenda inferior. . . . . 123 


\section{Sumário}

1. Introdução . . . . . . . . . . . . . . . . . . . . . . . . . . . 17

1.1 As Nebulosas Planetárias . . . . . . . . . . . . . . . . . . . . . 17

1.1.1 Uma perspectiva histórica . . . . . . . . . . . . . . . 17

1.1.2 Características do espectro . . . . . . . . . . . . . . . . 19

1.1.3 Morfologia . . . . . . . . . . . . . . . . . . . 21

1.1.4 Sub-estruturas. . . . . . . . . . . . . . . . . 22

1.2 Códigos de fotoionização . . . . . . . . . . . . . . . . . . 23

1.3 Proposta do trabalho . . . . . . . . . . . . . . . . . . . . . . . . 24

2. Observações . . . . . . . . . . . . . . . . . . . . . . . . 27

2.1 Dados observacionais . . . . . . . . . . . . . . . . 27

2.2 Configuração instrumental . . . . . . . . . . . . . . . . . . . 28

2.2.1 Espectroscopia de fenda longa . . . . . . . . . . . . . . . . . 28

2.2.2 Espectroscopia de campo integral . . . . . . . . . . . . . 29

2.3 Redução dos dados observacionais . . . . . . . . . . . . . . . . . . . . . . 30

2.3.1 Correção de avermelhamento . . . . . . . . . . . . . . . . . 33

2.4 Cálculo dos parâmetros físicos . . . . . . . . . . . . . . . . . . 34

2.4.1 Densidade eletrônica . . . . . . . . . . . . . . . . . 34

2.4.2 Temperatura eletrônica . . . . . . . . . . . . . . . . . 36

2.4 .3 Abundâncias iônicas . . . . . . . . . . . . . . . . . . 38

2.4.4 Abundâncias químicas . . . . . . . . . . . . . . . . . 39

2.4.5 Diagramas de diagnóstico do plasma . . . . . . . . . . . . . . . 40 
3. Simulações . . . . . . . . . . . . . . . . . . . . . . . . . . 43

3.1 Cloudy e Cloudy_3D . . . . . . . . . . . . . . . . . . . 43

3.2 Parâmetros e vínculos das simulações . . . . . . . . . . . . . . . . . . . 45

3.2.1 Fonte central . . . . . . . . . . . . . . . . . 46

3.2.2 Geometria e perfil de densidade . . . . . . . . . . . . . . . . . 47

3.2.3 Composição química . . . . . . . . . . . . . . . . . . . . . . . 48

3.3 Erros estimados e incertezas envolvidas . . . . . . . . . . . . . . . . . . 48

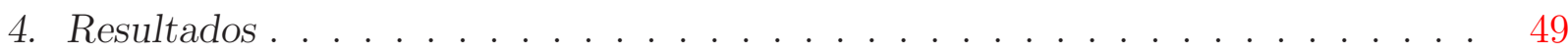

4.1 Espectroscopia de fenda longa . . . . . . . . . . . . . . . . . . . . . 49

$4.1 .1 \quad \mathrm{IC} 4406 \ldots \ldots \ldots \ldots . \ldots \ldots . \ldots \ldots$

$4.1 .2 \quad \mathrm{NGC5844} \ldots \ldots \ldots \ldots \ldots 5$

4.2 Espectroscopia de campo integral . . . . . . . . . . . . . . . 63

$4.2 .1 \quad$ NGC6572 . . . . . . . . . . . . . . . . . . . . 63

$4.2 .2 \quad \mathrm{IC} 2553 \ldots \ldots \ldots \ldots$

$4.2 .3 \quad \mathrm{NGC5882} \ldots \ldots \ldots \ldots \ldots . \ldots \ldots \ldots$

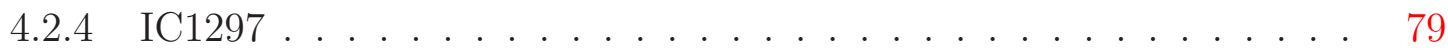

4.2 .5 IC4776 . . . . . . . . . . . . . . . . . 83

4.3 Diagramas de diagnóstico f . . . . . . . . . . . . . . . . 87

5. Conclusões. . . . . . . . . . . . . . . . . . . . . . . . . . 91

5.1 Perspectivas . . . . . . . . . . . . . . . . . . . . . . 94

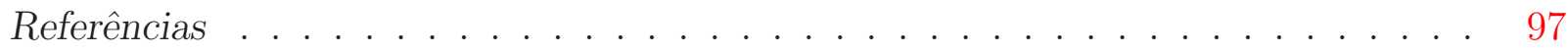

Apêndice

A. O script de redução PNPACK . . . . . . . . . . . . . . . . . . . . . 105

B. Dados observacionais do projeto . . . . . . . . . . . . . . . . 109

C. Tabela de fluxos obtidos com espectroscopia de fenda-longa . . . . . . . . . . . . 111 
Capítulo 1

\section{Introdução}

\subsection{As Nebulosas Planetárias}

\subsubsection{Uma perspectiva histórica}

Com o aprimoramento do telescópio no século XVIII, evidenciou-se a existência de dois tipos de objetos na esfera celeste: os pontuais (ou estelares), em oposição aos extensos, chamados também de "nebulosos". O primeiro catálogo sistemático de objetos extensos é de Charles Messier, em 1784.

Quase um século depois, o astrônomo William Herschel também se dedicou às observações de objetos extensos. É atribuído a ele o termo "nebulosa planetária" para a descrição deste tipo de objeto, similares a discos de coloração esverdeada, e que lembravam um planeta visto com a instrumentação da época.

Em 1864, William Huggins foi o primeiro a observar as linhas espectrais numa Nebulosa Planetária (daqui em diante, NP). Tipicamente, o espectro de uma NP apresenta somente fortes linhas em emissão, posteriormente identificados como a desexcitação radiativa na estrutura do átomo. Num primeiro momento, um grande número de linhas espectrais de NPs não foi identificado com as linhas de laboratório. Para explicar as fortes linhas de emissão na região correspondente ao verde do espectro foi sugerida a existência de um elemento químico presente nestes objetos denominado "nebulium".

Com a consolidação do conhecimento dos elementos químicos e os avanços da mecânica quântica, em 1928 o astrônomo Ira Sprague Bowen identificou oito das linhas nebulares intensas como resultadas de estados metaestáveis dos elementos Nitrogênio e Oxigênio. A hipótese do nebulium foi descartada, consolidando-se a descrição das NPs como um gás 
ionizado de baixa densidade.

Uma compreensão mais completa das NPs só ocorreu na segunda metade do século XX, quando estas foram analisadas no contexto do diagrama de Hertzsprung-Russell. O astrônomo I. Shklovsky, em 1956, sugere que as NPs são progenitoras das estrelas anãobrancas, descendentes de gigantes vermelhas. Abell e Goldreich (1966) corroboraram esta visão ao comparar as velocidades de expansão típicas da NPs com as velocidades de escape de gigantes vermelhas, sugerindo que o material ejetado da atmosfera destas estrelas formaria as NPs. Estes autores também estimaram através da quantidade de NPs esperadas para a Galáxia e seu curto tempo de vida $\left(2 \times 10^{4}\right.$ anos $)$, que praticamente todas as estrelas de baixa massa passariam pelo estágio de NP. Isto ilustra a importância das NPs no cenário de evolução estelar.

Assim, contemporaneamente as Nebulosas Planetárias são atribuídas a um dos estágios finais da evolução estrelas de baixa e intermediária massa $\left(0,8 \mathrm{M}_{\odot}\right.$ a $\left.8 \mathrm{M}_{\odot}\right)$, sendo formadas pela fotoionização do material ejetado pela estrela central que passou pela fase de gigante. As NPs apresentam distintos tamanhos e morfologia, bem como propriedades físicas (densidades, temperaturas, abundâncias, etc). As principais propriedades físicas são apresentadas na tabela 1.1, adaptada de Cazetta (1999).

Tabela 1.1 - Principais propriedades das Nebulosas Planetárias

\begin{tabular}{cc}
\hline \hline \multicolumn{2}{c}{ Nuvem } \\
\hline Densidade & $10^{2}-10^{5} \mathrm{~cm}^{-3}$ \\
Temperatura eletrônica & $5000-20000 \mathrm{~K}$ \\
Velocidade de expansão & $4-60 \mathrm{~km} / \mathrm{s}$ \\
Dimensão & $0,005-1 \mathrm{pc}$ \\
\hline Fonte Central \\
\hline Temperatura & $25000-300000 \mathrm{~K}$ \\
Luminosidade & $10-10^{4} \mathrm{~L}_{\odot}$ \\
Massa & $<1,4 \mathrm{M}_{\odot}$ \\
\hline
\end{tabular}

A partir de uma classificação das abundâncias químicas das Nebulosas Planetárias, Peimbert (1978) definiu 4 tipos quimicamente distintos de NPs e os situou no cenário 
evolutivo da Galáxia. Desde então, as NPs são estudadas como um importante vínculo nas teorias de evolução estelar, propriedades da Galáxia, de outras galáxias, e a evolução química destes sistemas.

\subsubsection{Características do espectro}

Diferentemente das estrelas, que apresentam um espectro contínuo, o espectro óptico das NPs é dominado por linhas de emissão que resultam do decaimento eletrônico nos átomos ou íons.

Nas estrelas, elétrons em um átomo estão distribuídos por muitos níveis de energia em virtude da alta densidade de partículas e radiação. Elétrons ligados são excitados ou por elétrons livres colidindo com o átomo, ou pela absorção de um fóton. A emissão resultante de tais interações resulta num espectro que se aproxima muito à de um corpo negro. Já no meio interestelar, ambiente das NPs, ambas as densidades de partículas e radiação são baixas, e a distribuição populacional dos elétrons ligados pode estar longe da condição de equilíbrio termodinâmico dados pela equação de Boltzmann.

Tradicionalmente, a discussão sobre a física das NPs parte do modelo estático clássico. Assume-se que as NPs são constituídas por duas componentes: uma estrela central e uma nebulosa gasosa circundante. Como a estrela é quente o suficiente, muito de sua energia será emitida no ultravioleta (UV). Estes fótons UV serão capazes de ionizar os átomos presentes na nebulosa. Os elétrons ejetados no processo de ionização transportarão energia cinética para a excitação colisional de átomos pesados (carbono, nitrogênio, oxigênio, etc.). E emissões espontâneas de vários estados excitados de diferentes átomos e íons são responsáveis pelo rico espectro de linhas de emissão observável neste tipo de objeto.

A energia típica de separação entre estados eletrônicos de muitos dos átomos pesados é da ordem de 1 elétron-Volt $(e V)$, correspondendo a fótons na parte visível ou UV do espectro. O único fundo contínuo do visível ou do UV disponível no meio interestelar é o da luz estelar difusa, que em geral não é forte o suficiente para que excitação por absorção estimulada seja significativa. Assim, os mecanismos para que o elétron ligado possa ser encontrado em um estado excitado são através de excitação colisional a partir de um estado mais baixo, ou como consequência da recombinação entre um elétron livre e íons. Os fótons de linha emitidos em consequência da recombinação do elétron com o íon dão origem às 
chamados linhas de recombinação. Hidrogênio e Hélio, com grandes intervalos de energia entre o primeiro estado excitado e o estado fundamental, são difíceis de se excitarem por colisões. Considerando os átomos pesados, cujas estruturas eletrônicas são mais complexas, com frequência eles possuem estados excitados com diferenças de energia de frações de $\mathrm{eV}$ acima do estado fundamental e podem ser mais eficientemente excitados por colisões.

As regras de seleção de transições eletromagnéticas por acoplamento LS ("LS coupling") são $\Delta L= \pm 1, \Delta S=0$ e $\Delta J=0, \pm 1$ exceto $J=0 \rightarrow 0$. Transições que violam as regras de seleção do acoplamento LS podem ocorrer como transições de quadripolo elétrico ou dipolo magnético, mas seus coeficientes de emissão espontânea são muito menores que aqueles das transições de dipolo elétrico permitidas. Nas condições terrestres, a alta frequência de colisões implica que estados de mais alta energia serão colisionalmente desexecitados antes que tenham uma chance de decair radiativamente. Contudo, nas condições interestelares, o tempo entre colisões pode ser mais longo que o tempo de vida de um estado excitado, permitindo que estados mais altos decaiam pela emissão de um fóton de linha proibida, ou seja, que viola as regra de transição.

Muitas das linhas mais fortes das NPs são linhas proibidas. Por exemplo, as transições do OII e do SII na configuração $n p^{3}$ são proibidas porque envolvem transições dentro da mesma configuração e portanto violam a regra de seleção $\Delta l= \pm 1$. Linhas deste tipo são indicadas por colchetes (ex. [OII]).

O primeiro estado excitado do Hidrogênio (H) está em 10, $2 \mathrm{eV}$ acima do estado fundamental, correspondendo a uma temperatura de excitação $\frac{E}{k}$ de $\sim 10^{5} \mathrm{~K}$. Isto é muito mais alto que as temperaturas cinéticas típicas de $10^{4} \mathrm{~K}$ encontradas em NPs. Mesmo para elétrons com energia alta o suficiente para transpor a diferença de energia, as baixas densidades nas NPs implicam que a taxa de excitação ou ionização por colisão eletrônica seja muito menor que a taxa de emissão espontânea, permanecendo um elétron excitado colisionalmente no estado excitado por um tempo muito curto. Como o resultado, a população de um estado excitado do H é determinada não por excitação colisional a partir do estado fundamental, mas pela recombinação entre prótons e elétrons livres e a subseqüente cascata eletrônica por emissão espontânea. A ionização e os estados de excitação do átomo de $\mathrm{H}$ podem portanto serem assumidos como majoritariamente controlados por processos radiativos. 
Embora as abundâncias dos metais sejam muito menores que a do Hidrogênio, linhas atômicas excitadas colisionalmente nas NPs são tão fortes quanto as linhas de recombinação do $\mathrm{H}$ porque os processos colisionais são muitas ordens de magnitude mais rápidos que o processo de recombinação. Excitação colisional para estados metaestáveis mais baixos e seus subseqüentes decaimentos através de transições de dipolo magnético ou quadrupolo elétrico levam à emissão das linhas que são comumente referidas como linhas proibidas.

Apesar das NPs serem bem conhecidas por suas fortes linhas proibidas de íons como $\mathrm{N}^{+}, \mathrm{O}^{+}, \mathrm{O}^{2+}, \mathrm{S}^{+}, \mathrm{S}^{2+}, \mathrm{Ne}^{2+}, \mathrm{Ar}^{2+}$, as linhas proibidas não são as únicas linhas excitadas colisionalmente no espectro das NPs. Consideráveis linhas permitidas de metais no UVpróximo são também excitadas colisionalmente e são destacadas em alguns espectros de NPs.

\subsubsection{Morfologia}

A morfologia observada no imageamento é tradicionalmente usada como ferramenta para a classificação das Nebulosas Planetárias. Esta classificação tem como principal objetivo o conhecimento sobre formação e evolução desses objetos.

O modelo mais aceito para explicar a morfologia das NPs é o modelo de ventos interagentes, proposto por Kwok et al. (1978). Neste modelo, a estrutura das NPs decorre da interação de um vento rápido de baixa densidade proveniente da estrela central com o remanescente de um vento lento e denso da gigante AGB.

A partir deste modelo, utilizando-se de cálculos hidrodinâmicos, Balick (1987) apresenta um modelo sucinto de como os ventos interagentes dariam origem às diferentes morfologias observadas nas NPs. No esquema proposto pelo autor (figura 1.1 (Balick, 1987)), uma NP basicamente pode assumir três tipos de morfologia (tridimensional):

- Esférica

- Elipsóide (prolato)

- Bipolar (ou "ampulheta")

Dependendo de condições de projeção com a linha de visada do observador, uma imagem bidimensional de uma NP não é capaz de determinar sua morfologia (estrutura tridimensional). Para se definir inequivocamente a distribuição tridimensional de matéria de uma 


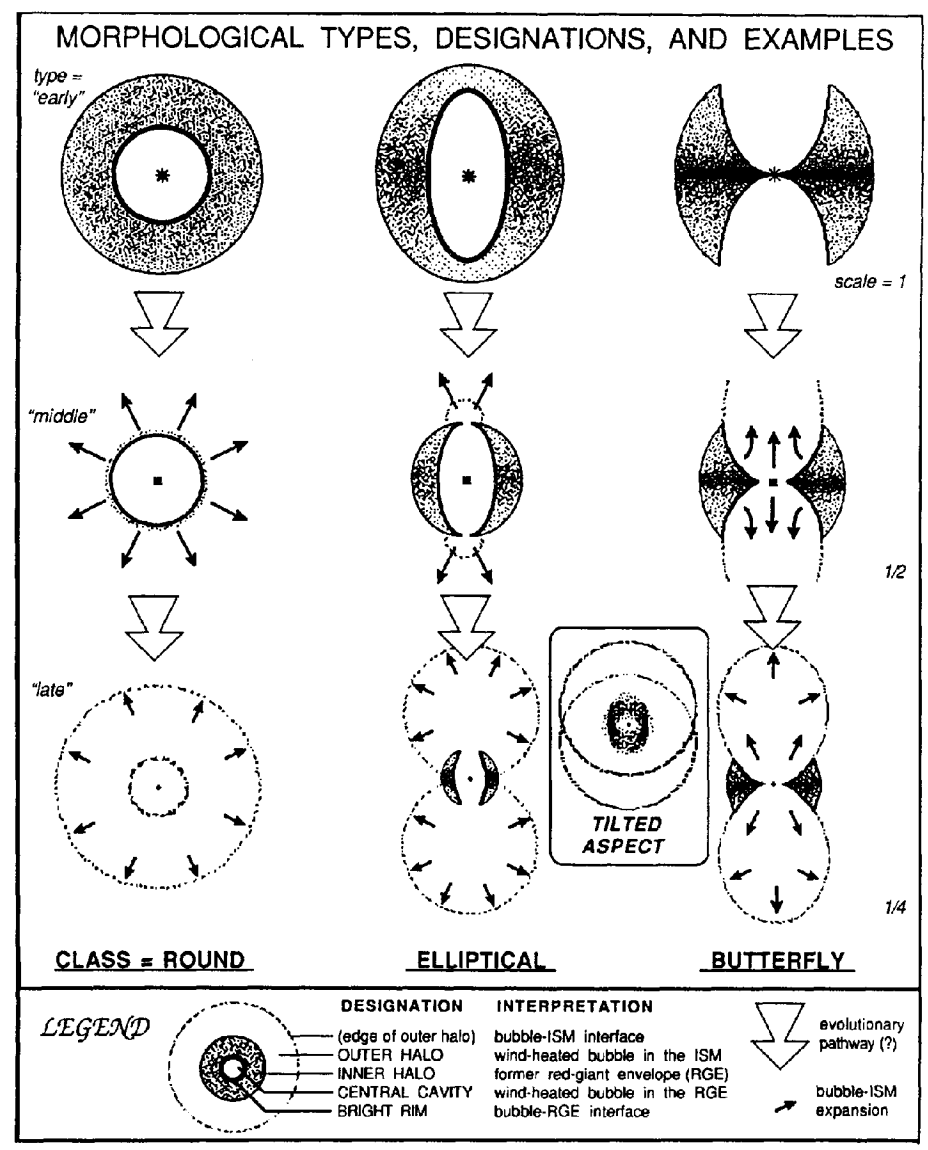

Figura 1.1: Ilustrações bidimensionais das diferentes morfologias assumidas por uma Nebulosa Planetária.

NP, são necessários dados observacionais detalhados de sua estrutura angular, vinculados à simulações de regiões ionizadas e suas respectivas projeções (Monteiro, 2000).

Somente com a determinação da estrutura tridimensional da NP pode-se obter sua correta classificação morfológica, além de permitir obter de uma maneira consistente os valores das propriedades observadas.

\subsubsection{Sub-estruturas}

O advento do imageamento em alta resolução com o telescópio Hubble, e posteriormente com os telescópios da categoria de 8-10 metros, permitiu a execução de imagens sem precedentes de objetos difusos como as nebulosas planetárias. Uma das características mais notáveis mostradas por tais imagens é a riqueza de detalhes das estruturas associadas com as nebulosas bipolares. 
Além das componentes principais que definem sua geometria (seção 1.1.3) e a eventual presença de um halo formado por um gás difuso, muitas NPs apresentam estruturas de menor escala que são proeminentes em linhas de baixa ionização. Assim, são comumente denominados por estruturas de baixa-ionização (EBI ou LIS, em inglês). Elas possuem distintas propriedades morfológicas e cinemáticas na forma de pares de nós, filamentos, jatos ou características isoladas que podem ser substancialmente diferentes das características da componente principal.

Além de se destacarem no imageamento de alta resolução, estas estruturas podem ser espectroscopicamente identificadas desde que tem uma resolução angular adequada. Um estudo detalhado destas sub-estruturas é feito por Gonçalves et al. (2001).

\subsection{Códigos de fotoionização}

Dado o avanço computacional da últimas décadas, é crescente a utilização de modelos numéricos de fotoionização no estudo de objetos astrofísicos. Incorporando os cálculos envolvidos na transferência radiativa, na interação por diversos processos da radiação com matéria, e utilizando dados atômicos de precisão, estes códigos fornecem uma descrição robusta do objeto, desde as condições físicas de seu material constituinte à descrição da radiação transmitida e emitida.

Os códigos de fotoionização de uma maneira geral partem de três hipóteses básicas: i)estado estacionário do sistema; ii) equilíbrio de ionização; iii) equilíbrio térmico. A hipótese de estado estacionário implica em desprezar processos dinâmicos que poderiam ocorrer com o material (expansão, por exemplo). Assumida esta hipótese, pode-se determinar a temperatura e o grau de ionização em cada ponto do objeto através das hipóteses de equilíbrio térmico e de ionização. Na verdade estas condições são acopladas, pois as taxas em que ocorrem os processos físicos relacionados ao equilíbrio de ionização são fortemente dependentes da temperatura, que por sua vez depende das abundâncias iônicas no gás (Monteiro, 2004).

A modelagem de um determinado objeto requer o uso de parâmetros de entrada no código que são provenientes de resultados observacionais, tais como as propriedades da fonte emissora e a distribuição espacial de temperaturas e densidades eletrônicas. Os códigos são então executados num processo iterativo de ajuste dos parâmetros de saída à 
estes vínculos observacionais.

Existem distintos códigos de fotoionização disponíveis. Optou-se neste projeto por usar-se o código de fotoionização Cloudy, que tem sido extensamente utilizado nas últimas décadas, está em contínuo aprimoramento e atualmente permite modelar a região emissora em grande detalhe. Ele divide a mesma num grande número de zonas nas quais as condições sejam aproximadamente constantes, e o nível de ionização (com a consequente emissão) é calculado levando-se em conta todos os processos de ionização e recombinação. Os processos de ionização incluem fotoionização, efeito Auger, ionização colisional e transferência de cargas. Os processos de recombinação incluem a recombinação radiativa, dieletrônica e transferência de carga, entre outros. Uma boa descrição dos processos físicos envolvidos no desenvolvimento dos códigos é feita por Ferland (2003). Maiores detalhes do código e sua utilização estão no capítulo 3.

\subsection{Proposta do trabalho}

Na maioria das vezes, a determinação do parâmetros físicos e de abundâncias química nas NPs é feita de forma média, ou seja, levando-se em conta os fluxos totais observados da NP. Apesar deste procedimento garantir certa homogeneidade no procedimento, não leva em conta as estruturas dos objetos. Os mesmos podem ter regiões de maior ou menor densidade, eventualmente com distintas composições químicas, além de um esperado gradiente de ionização a partir da estrela central.

O uso de telescópios de grande resolução angular permitiu mapear as estruturas bipolares de muitas nebulosas e examinar como as mesmas estão orientadas no céu, bem como suas dimensões. Com tais informações é possível utilizar-se telescópios de porte menor para que, com a fenda devidamente orientada em relação à nebulosa, se possa fazer espectroscopia de regiões específicas da mesma com o objetivo de se descobrir: i) a distribuição dos parâmetros físicos ao longo da nebulosa; ii) a distribuição das abundâncias químicas ao longo na nebulosa. Um exemplo deste tipo de trabalho é o estudo feito por Gonçalves et al. (2003), que através de espectroscopia de fenda longa sobre NGC7009, baseando-se nas imagens obtidas com o telescópio Hubble, derivaram os parâmetros físicos, mecanismos de excitação e propriedades químicas deste objeto. Um estudo análogo para K4-47 foi feito pelo mesmo grupo (Gonçalves et al., 2004), obtendo também excelentes resultados. 
A técnica de espectroscopia de campo integral também se mostra ideal para este tipo de análise, desde que possua uma cobertura adequada do céu. Através desta técnica, numa mesma observação pode ser investigado bidimensionalmente o espaço de parâmetros na NP, ao invés de obtê-los numa única direção como no caso de fenda longa. O inconveniente deste método está na adequada cobertura angular para as NPs extensas, em conjunto com uma boa cobertura espectral, além da dificuldade de uma calibração segura em fluxo. Os detalhes da configuração instrumental utilizada no trabalho estão no capítulo 2.

Os poucos objetos já estudados do modo descrito acima estão no hemisfério norte. No sul, onde se encontra a maioria das nebulosas planetárias, existe uma amostra expressiva de nebulosas bipolares, algumas das quais já com imageamento em alta resolução disponível, para as quais as únicas determinações de abundância disponíveis na literatura são as médias.

Com o presente projeto pretende-se determinar parâmetros físicos e abundâncias químicas para uma amostra de NPs extensas da Galáxia que tenham estrutura bipolar e, a partir destes resultados, modelar tais objetos através de um código de fotoionização. Novos dados observacionais foram coletados de uma amostra de nebulosas planetárias bipolares da Galáxia no OPD (LNA/MCT), usando o telescópio de $1.60 \mathrm{~m}$, e os resultados obtidos, combinados com outros dados colhidos da literatura, foram utilizados como parâmetros de entrada no código de fotoionização Cloudy (Ferland et al., 1998) em sua versão tridimensional (Morisset, 2006).

Desta forma, espera-se obter de uma maneira auto-consistente a descrição realista do objeto, vinculando suas observações com a simulações dos processos físicos que nele ocorrem. Esse processo permite a identificação de eventuais estruturas nebulares, bem como a obtenção precisa de seus parâmetros físicos e abundâncias químicas. 


\section{Capítulo 2}

\section{Observações}

\subsection{Dados observacionais}

As observações do projeto foram realizadas com o uso do telescópio Perkin-Elmer de 1,60m do Observatório Pico dos Dias (OPD), em missões observacionais entre 2007 e 2010. O observatório está subordinado ao Laboratório Nacional de Astrofísica do Ministério de Ciência de Tecnologia (LNA/MCT).

A amostra de Nebulosas Planetárias a ser observada foi definida segundo os critérios: i) objetos extensos, adequados a instrumentação; ii) objetos com alto brilho superficial; iii) objetos que exibem simetria espacial adequada às simulações.

Objetos extensos possuem ampla discussão de suas propriedades na literatura, e vários com imageamento também disponível. Para alguns alvos existem imagens em alta-resolução, provenientes de grandes telescópios $(8 m-10 m)$ e do telescópio espacial Hubble. O imageamento é um fator importante na análise da distribuição e eventuais mudanças dos parâmetros das NPs (ver seção 1.1.4). Destacamos que a análise química destes objetos em geral é feita com fluxos totais em uma fenda, evidenciando suas propriedades médias.

Com o objetivo de analisar as propriedades espaciais dos objetos angularmente resolvidos, o fluxo total emitido pelos mesmos por si só não garante observações de qualidade. O alto brilho superficial dos objetos é necessário para a otimização do uso do telescópio, garantindo integrações com bom sinal/ ruído para o objeto em toda a sua extensão. A informação de catálogos com fluxo de linha dos candidatos (tal como Acker et al. (1992)) foi utilizada para se priorizar alvos com maior fluxo relativo nas linhas de diagnóstico do plasma.

A simetria dos objetos é importante para se poupar tempo nas simulações. Deu-se 
preferência a objetos com simetria bipolar para a respectiva modelagem.

A técnica de espectroscopia de fenda longa com definição de variadas janelas de extração mostra-se adequada, pois para grandes objetos ( $\sim 1^{\prime}$ de diâmetro) pode-se cobrir em uma única exposição todo o objeto em uma de suas posições de fenda, com boa calibração do fluxo. Neste tipo de objeto observações em espectroscopia de campo integral somente seriam viáveis em grandes mosaicos, com imprecisões no posicionamento e também na calibração de fluxos. A tabela 2.1 apresenta os objetos observados com a técnica de espectroscopia de fenda longa em missões ao LNA e que podem ser utilizados para a dissertação. O espectrógrafo utilizado foi o Cassegrain ${ }^{1}$.

Tabela 2.1 - Objetos analisados neste trabalho com a técnica de espectroscopia de fenda longa

\begin{tabular}{ccccccc}
\hline \hline PNG & Nome & Integrações & Fendas & P.A. & Janelas & Diâmetro H $\alpha$ \\
\hline $317.1-05.7$ & NGC5844 & $2 x 1200 \mathrm{~s}$ & 3 & $0^{\circ}$ & $8,10,8$ & $73 ", 79 ", 73 ”$ \\
$319.6+15.7$ & IC4406 & $3 x 1200 \mathrm{~s}$ & 1 & $0^{\circ}$ & 13 & $111 "$ \\
\hline
\end{tabular}

Disponível no OPD, está também o espectrógrafo Eucalyptus ${ }^{1}$. Este consiste numa unidade de campo integral de grande cobertura angular para este tipo de instrumento $\left(15^{\prime \prime} \times 30^{\prime \prime}\right)$, mas de baixa resolução $\left(\sim 0,93^{\prime \prime}\right.$, num conjunto de 510 fibras). A cobertura angular torna o instrumento ideal para o estudo deste tipo de objeto, mapeando bidimensionalmente as propriedades do objeto numa única exposição. Os objetos analisados com esta técnicas estão na tabela 2.2.

\subsection{Configuração instrumental}

\subsubsection{Espectroscopia de fenda longa}

A técnica da espectroscopia de fenda longa é bastante conveniente para o mapeamento espacial destes objetos, já que em nebulosas bipolares existe um nítido eixo de simetria em função da qual foi posicionada a fenda do espectrógrafo. Os parâmetros referentes às observações estão na tabela 2.3.

\footnotetext{
${ }^{1} \mathrm{O}$ LNA/MCT tem em seu sítio eletrônico $h t t p: / / w w w . l n a . b r$ os manuais contendo a descrição da instrumentação.
} 
Tabela 2.2 - Objetos analisados neste trabalho com a técnica de espectroscopia de campo integral

\begin{tabular}{cccccc}
\hline \hline PNG & Nome & Data & $\lambda_{\text {central }}$ & Integrações & Diâmetros H $\alpha$ \\
\hline $002.0-13.4$ & IC4776 & $08 / 06 / 10$ & 6550 & $5 \times 900 \mathrm{~s}$ & 8 "x13" \\
$034.6+11.8$ & NGC6572 & $08 / 06 / 10$ & 6550 & $5 \times 450 \mathrm{~s}$ & 12 "x17"* \\
$285.4-05.3$ & IC2553 & $08 / 06 / 10$ & 6550 & $5 \times 1200 \mathrm{~s}$ & 5 "x9" \\
$327.8+10.0$ & NGC5882 & $08 / 06 / 10$ & 6550 & $5 \times 1200 \mathrm{~s}$ & 16 "x20" \\
$358.3-21.6$ & IC1297 & $08 / 06 / 10$ & 6550 & $3 \times 900 \mathrm{~s}$ & 13 "x16" \\
\hline
\end{tabular}

*Diâmetro esperado, uma vez que excedeu o campo.

A metodologia de análise consiste na derivação do resultados de parâmetros físicos e abundâncias químicas ao longo das regiões cobertas pelas fendas, de forma que os resultados são obtidos em distintos pontos de cada nebulosa, com particular atenção às variações dos parâmetros que sejam associadas com a presença de estruturas. O espaçamento entre as regiões foi fixado em 8,4" (15 pixels) a fim de garantir alto sinal/ruído no espectro observado e minimizar erros devido ao "seeing" e correções de traçado do espectro sobre o CCD.

Tabela 2.3 - Parâmetros da configuração observacional de fenda longa

\begin{tabular}{cc}
\hline \hline Escala espacial do detector & $0,56 " /$ pix \\
Largura da fenda & $1,5 "$ \\
Comprimento da fenda & $>5$, \\
Dispersão espectral & $\sim 2,2 \AA /$ pix \\
Cobertura espectral & $\sim 3600$ a $7800 \AA$ \\
\hline
\end{tabular}

\subsubsection{Espectroscopia de campo integral}

A espectroscopia de campo integral permite a obtenção de um espectro individual para cada elemento de resolução na área do céu observado, através de uma matriz de fibras ópticas (unidade de campo integral, UCI ou IFU em inglês). Isso torna extremamente interessante a observação de NPs, uma vez que suas propriedades bidimensionais podem 
ser diretamente mensuradas ao longo de toda a sua extensão (com um objeto de dimensões angulares adequadas). As características das observações de campo integral estão na tabela 2.4 .

Contudo, calibrações em fluxo tornam-se complicadas para este tipo de instrumento pois a resposta à radiação incidente é diferente para cada fibra óptica, mesmo na hipótese de iluminação uniforme pelo telescópio em toda a área da matriz de fibras. Outra característica do espectrógrafo Eucalyptus é de possuir pequena cobertura espectral. Com isso, não se pode obter numa única exposição os fluxos $\mathrm{H} \alpha / \mathrm{H} \beta$ para a correção do avermelhamento interestelar, bem como avaliar diretamente a razão de muitas das linhas de emissão com a linha $\mathrm{H} \beta$.

Tabela 2.4 - Parâmetros da configuração observacional de campo integral

\begin{tabular}{lc}
\hline \hline Cobertura espacial total & $15 \times 30 "$ \\
Número de fibras & 510 \\
Área coberta por cada fibra & $0,93 "$ \\
Dispersão espectral & $\sim 0,3 \AA /$ pix \\
Cobertura espectral & $\sim 630 \AA$ \\
\hline
\end{tabular}

\subsection{Redução dos dados observacionais}

Podemos dividir as etapas da redução dos espectros obtidos nas missões observacionais nos seguintes processos:

1. Correções iniciais das leituras do CCD.

2. Extração dos espectros de interesse.

3. Calibração em comprimento de onda.

4. Calibração em fluxo.

5. Correção da extinção interestelar.

6. Cálculo dos fluxos das linhas de interesse. 
A redução dos dados é feita com o conjunto de pacotes $N O A O$, parte do IRAF ${ }^{2}$. Abaixo, listamos os principais processos realizados sobre os dados nesta etapa da redução e com base nas rotinas (ou "tasks") IRAF relacionadas.

1. ccdproc realiza as seguintes correções de leitura:

$B I A S$ - Imagens bias são obtidas com o obturador da câmera fechado, para verificação do ruído de leitura resultante do descarregamento do CCD (flutuação do tipo Poisson). Tira-se em média de 10 a 20 imagens de curta exposição para adequada estimativa da média. Estas imagens caracterizam-se por baixas contagens e pequena variação pixel a pixel. São combinadas com a rotina zerocombine.

FLAT - Imagens flat são imagens obtidas com lâmpadas incandescentes intensas, para medir a resposta do detector à radiação incidente. Em imagens bidimensionais, elas permitem avaliar a resposta de cada pixel a uma mesma luminosidade. No caso de espectroscopia, as imagens flat avaliam também a resposta do detector à emissão de corpo negro da lâmpada, com contagem proporcionais à eficiência da instrumentação em cada comprimento de onda. As imagens são combinadas com a rotina flatcombine.

Uma correção complementar a este tipo de imagem em espectroscopia consiste em realçar a resposta local (de alta frequência) do CCD. Para isto, deve-ser descontar a variação das intensidades ao longo do espectro (resposta de baixa frequência), normalizando-o. O espectro combinado é normalizado com a rotina imarith, após ter as variação de alta frequência suavizadas com a rotina boxcar. Ambas as rotinas estão incluídas no próprio IRAF.

TRIM - Recorte das extremidades do CCD, que apresentam baixa contagem de fótons. Desta forma, aprimora-se as correções que ajustam toda a área do CCD, como a correção de flat.

2. apall Na imagem bidimensional do CCD, marca-se a extensão de interesse do objeto, chamada abertura (eixo espacial). Integra-se a contagem em cada intervalo de extração definido ao longo da fenda, descontando as contagens de fundo do céu.

\footnotetext{
2 IRAF é um conjunto de programas voltados para a redução de dados astronômicos. Mais informações no site http://iraf.noao.edu.
} 
3. identify A partir das linhas de emissão conhecidas das lâmpadas de calibração (lâmpadas de He-Ar ou Ne-Ar), obtêm-se a função dos pixels por comprimento de onda, que então é aplicada nos espectros das aberturas das NPs.

4. sensfunc Compara as contagens obtidas com o fluxo tabelado das estrelas padrões, estabelecendo um função de contagem por unidade de fluxo. observatory Configura o local geográfico do observatório para correção da extinção média pela massa de ar. Quanto mais próximo ao zênite, menor massa de ar e consequentemente menor extinção atmosférica.

Na redução dos dados de espectroscopia de campo integral, além das imagens flat de cúpula, recomenda-se avaliar a resposta do CCD sem a instrumentação, iluminando-o diretamente. Deve-se notar que as correções são feitas individualmente para cada fibra até o passo de calibração em comprimento de onda. A calibração em fluxo torna-se complicada, pois não se pode iluminar todas as fibras com uma fonte padrão, nem garantir que fração do fluxo de uma estrela padrão seria observada por uma das fibras.

Todas estas correções foram integradas num script de redução denominado PNPACK. Além das correções anteriormente listadas, o script também faz a correção da extinção interestelar (ver seção 2.3.1), o cálculo dos fluxos das linhas de emissão por ajuste gaussiano e, eventualmente, separação de linhas ( "deblend") com a rotina splot. A obtenção dos fluxos por ajuste é de suma importância, uma vez que existe uma dispersão sobre os pixeis do detector, proporcional a intensidade da linha e das condições de observação. A descrição do script é feita no apêndice A.

Dada a impossibilidade de aplicar de uma forma robusta a função-sensibilidade para todas as fibras da UCI, optou-se por trabalhar com razões de fluxos de linhas para a análise dos resultados. Na cobertura espectral utilizada neste trabalho (de $6.250 \AA ̊$ à $6.650 \AA$ ), três conjuntos de linhas foram selecionados para análise dos objetos e vínculo com os modelos, como está detalhado na seção 2.4.5: i) $[\mathrm{NII}](\lambda 6548+6584) / \mathrm{H} \alpha$, ii) $[\mathrm{SII}](\lambda 6716 / \lambda 6731)$ e iii) $\log [\mathrm{SIII}](\lambda 6312) /[\mathrm{SII}](\lambda 6725)$. A letra $\lambda$, a ser utilizada de agora em diante, designa comprimento de onda em Ångstroms $(\AA)$.

Nos dois primeiros casos, como a diferença entre valores do comprimento de onda é muito pequeno $(\Delta \lambda \sim 15 \AA)$, assumimos que os efeitos da extinção interestelar e da diferença de eficiência instrumental sejam desprezíveis. No terceiro caso, convencionando 
$\lambda 6725=\lambda(6716+6731)$, e sendo $\alpha_{\lambda}$ o fator de correção do fluxo observado devido a extinção interestelar e eficiência instrumental para o comprimento de onda $\lambda$, temos:

$$
\frac{I(\lambda 6312)}{I(\lambda 6725)}=\frac{F(\lambda 6312)}{F(\lambda 6725)} \frac{\alpha_{6312}}{\alpha_{6725}} .
$$

Uma vez que o fluxo das linhas é obtido com subtração de emissões do contínuo, a correção na razão dos fluxos devido à extinção interestelar e eficiência instrumental é um termo proporcional aos mesmos. Em escala logarítmica, temos:

$$
\log \frac{I(\lambda 6312)}{I(\lambda 6725)}=\log \frac{F(\lambda 6312)}{F(\lambda 6725)}+\log \gamma
$$

Onde $\gamma=\alpha_{6312} / \alpha_{6725}$, constante, determinada se conhecidos os coeficientes da extinção interestelar e da diferença de eficiência instrumental. Assim, se fixarmos um valor médio para o qual ocorrem as variações dos fluxos nos modelos e nas observações, as razões $\log I(\lambda 6312) / I(\lambda 6725)$ terão as mesmas variações que $\log F(\lambda 6312) / F(\lambda 6725)$ observadas.

\subsubsection{Correção de avermelhamento}

A fim de se obter a intensidade dos fluxos relativos efetivamente emitidos pelos objetos, é necessário aplicar sobre os fluxos observados a correção da chamada extinção interestelar. Esta extinção que ocorre no meio interestelar é fortemente seletiva em função do comprimento de onda da radiação transmitida.

Esta extinção é comumente expressa em termos de excesso de cor $E\left(\lambda_{1}-\lambda_{2}\right)$ :

$$
E\left(\lambda_{1}-\lambda_{2}\right)=A_{\lambda_{1}}-A_{\lambda_{2}}=2,5 \log \frac{I\left(\lambda_{1}\right)}{I\left(\lambda_{2}\right)} \frac{F\left(\lambda_{2}\right)}{F\left(\lambda_{1}\right)}
$$

A função de avermelhamento utilizada no trabalho é a obtida por Fitzpatrick (1999), que por razões históricas é expressa em termos do excesso de cor E(B-V), em magnitudes dos filtros B e V:

$$
\begin{aligned}
{\left[\frac{A_{\lambda}}{E(B-V)}\right]=} & 0.00001+0.22707 x+1.95243 x^{2}-2.67596 x^{3} \\
& +2.6507 x^{4}-1.26812 x^{5}+0.27549 x^{6}-0.02212 x^{7}
\end{aligned}
$$

$\operatorname{com} x=1 / \lambda\left[\mu m^{-1}\right]$. Substituindo a equação 2.4 na equação $2.3, \operatorname{com} \lambda_{2}=\mathrm{H} \beta$ e isolando a razão de fluxo esperado:

$$
2,5 \log \left[\frac{I(\lambda)}{I(H \beta)}\right]_{\text {teorico }}=2,5 \log \left[\frac{F(\lambda)}{F(H \beta)}\right]_{\text {observado }}+E(B-V)\left[\frac{A_{\lambda}-A_{H \beta}}{E(B-V)}\right]
$$


onde $I(\lambda)$ é o fluxo na frequência de $\lambda$ na ausência de extinção e $F(\lambda)$ é o fluxo observado atenuado pela extinção.

Outra forma de medir a extinção interestelar é através do chamado parâmetro de extinção $c_{H \beta}$ (ou somente $c$ ):

$$
c_{H \beta}=\log \frac{I(H \beta)}{F(H \beta)}=\frac{A_{H \beta}}{2,5},
$$

Desta forma, temos substituindo os valores da equação 2.4:

$$
\begin{aligned}
c_{H \beta} & =0,4 E(B-V) A_{H \beta} \\
& =1,4463 \times E(B-V)
\end{aligned}
$$

O parâmetro de extinção também pode ser definido da forma:

$$
\begin{aligned}
C_{H \beta} & =\ln \frac{I(H \beta)}{F(H \beta)}, \\
& =2,3026 \times c_{H \beta}
\end{aligned}
$$

Em NPs, a expressão 2.5 é aplicada sobre o decremento de Balmer teórico para átomos de Hidrogênio nas condições físicas típicas de Nebulosas Planetárias (Osterbrock, 1989). Operacionalmente, os valores observados da razão $\mathrm{H} \alpha / \mathrm{H} \beta$ são comparados com o valor teórico de 2,85 esperado para uma NP com densidade de $5000 \mathrm{e}^{-} / \mathrm{cm}^{3}$ e temperatura eletrônica de $10000 \mathrm{~K}$ :

$$
0,4 E(B-V)\left[\frac{A_{H \alpha}-A_{H \beta}}{E(B-V)}\right]=\log \left[\frac{I(H \alpha)}{I(H \beta)}\right]_{\text {teorico }}-\log \left[\frac{F(H \alpha)}{F(H \beta)}\right]_{\text {observado }},
$$

substituindo os valores numéricos, $\operatorname{com} A_{H \alpha}$ e $A_{H \beta}$ calculados com a expressão 2.4, temos:

$$
E(B-V)=1,916 \log \left[\frac{F(H \alpha) / F(H \beta)}{2,85}\right]
$$

\subsection{Cálculo dos parâmetros físicos}

\subsubsection{Densidade eletrônica}

A densidade eletrônica, número de elétrons livres por unidade de volume, pode ser determinada pela razão da intensidade de certas linhas proibidas. Para a estimativa da densidade, é necessário avaliar a razão de linhas que tenham configuração externa $n p^{3}$, como o dubleto ${ }^{2} D$ do [OII] e do [SII] (ver figura 2.1, adaptada de Kwok (2000)). 


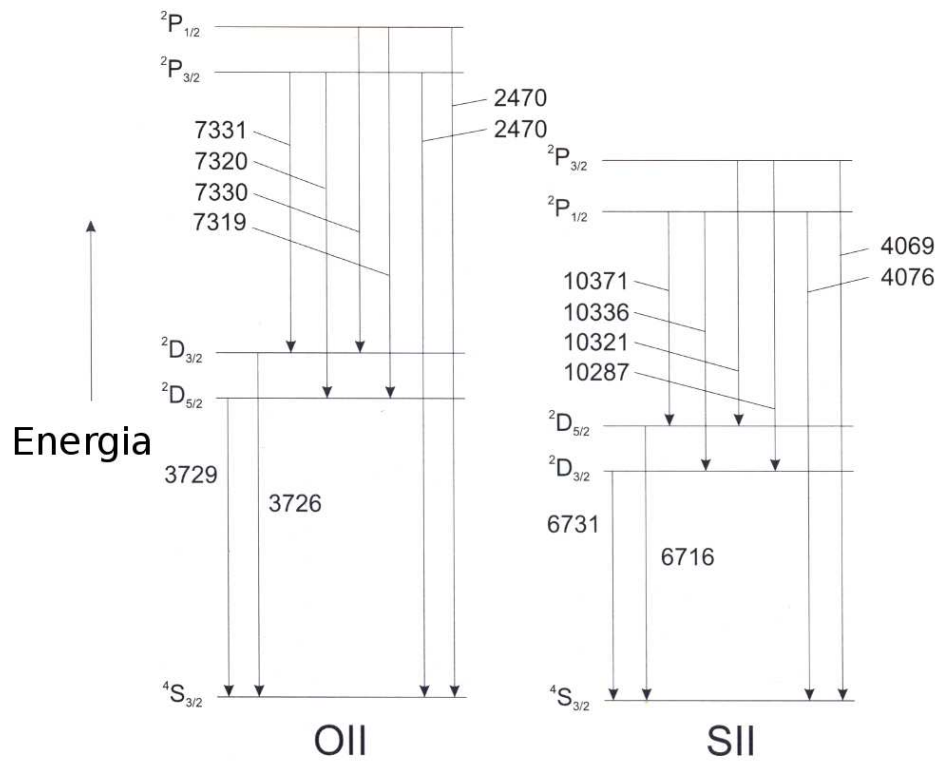

Figura 2.1: Os diagramas de energia de [OII] e [SII]. Os comprimentos de onda das transições são indicados em Å.

A explicação para a sua utilização é que em níveis de energia tão próximos $\left({ }^{2} D_{3 / 2}\right.$ e $\left.{ }^{2} D_{5 / 2}\right)$ a energia necessária para levar um elétron a qualquer um dos níveis superiores desde este estado de dubleto é praticamente a mesma, e a dependência com a temperatura eletrônica tem efeitos secundários. Sem a presença da desexcitação colisional, o valor da razão das intensidades do dubleto representa a razão entre as probabilidades de decaimento. Se considerada a desexcitação por colisões, então a emissão de cada linha dependerá de quão próxima está a densidade eletrônica da região emissora de sua densidade crítica (definida como a densidade para a qual a taxa de desexcitação colisional é igual a taxa de desexcitação radiativa). Para um intervalo de $10^{2} \mathrm{~cm}^{-3} \leq n_{e} \leq 2.10^{4} \mathrm{~cm}^{-3}$, a razão das linhas dos íons citados é muito sensível à densidade eletrônica.

A seção de choque colisional $\sigma_{j i}$ pode ser expressa da seguinte forma, usando a quantidade adimensional chamada de força colisional $\Omega_{j i}$ (Kwok, 2000):

$$
\begin{aligned}
\sigma_{j i}(v) & =\left(\pi a_{0}^{2}\right)\left(\frac{h R}{1 / 2 m_{e}^{2} v^{2}}\right) \frac{\Omega_{j i}}{g_{j}} \\
& =\left(\frac{\pi h^{2}}{4 \pi^{2} m_{e}^{2} v^{2}}\right) \frac{\Omega_{j i}}{g_{j}},
\end{aligned}
$$

onde $a_{0}$ o raio de Bohr, $g_{j}$ o fator de Gaunt, $R=2 \pi^{2} e^{4} m_{e} / h^{3}$ é a constante de Rydberg, $m_{e}$, e e $v$ a massa, carga e velocidade do elétron, respectivamente. Assim, a taxa de 
excitação colisional para um conjunto de elétrons à temperatura $T_{e}$ pode ser obtida pela média sobre uma distribuição Maxwelliana:

$$
C_{j i}\left(T_{e}\right)=\int_{v_{\text {min }}}^{\infty} v \sigma_{j i}(v) f(v) d v,
$$

onde $v_{\text {min }}=\left(2 E_{j i} / m_{e}\right)^{1 / 2}$.

Seja $A_{j i}$ o coeficiente de emissão espontânea. Assumindo uma configuração de dois níveis e radiação de fundo desprezível (tal que os termos de emissão estimulada possam ser omitidos), a equação de equilíbrio estatístico é:

$$
n_{2}\left(A_{21}+n_{e} C_{21}\right)=n_{1} n_{e} C_{12},
$$

cuja solução é:

$$
\frac{n_{2}}{n_{1}}=\frac{n_{e} C_{12}}{A_{21}}\left[\frac{1}{1+n_{e} C_{21} A_{21}}\right] .
$$

E portanto temos para a razão das intensidades:

$$
\begin{aligned}
\frac{I(\lambda 6716)}{I(\lambda 6731)} & =\frac{n_{3} A_{31}}{n_{2} A_{21}} \\
& =\frac{C_{13}}{C_{12}}\left(\frac{1+n_{e} C_{21} / A_{21}}{1+n_{e} C_{31} / A_{31}}\right),
\end{aligned}
$$

Se a densidade de elétrons é muito maior que a densidade crítica, ao invés do íon excitado emitir um fóton para retornar ao estado fundamental, o nível é desexcitado por uma colisão, perdendo-se a informação do nível daquele íon.

Cabe destacar que na maioria das nebulosas planetárias o nível de ionização é tão alto que a emissão do [SII] e mesmo do [OII] são provenientes de zonas de baixa ionização localizadas nas regiões mais externas do gás, ou nas partes mais densas onde a recombinação reduz a ionização. Por isso, tais densidades podem não ser representativas para a densidade em todo o volume da nebulosa.

\subsubsection{Temperatura eletrônica}

A configuração dos níveis de energia de determinados íons (figura 2.2, adaptada de Kwok (2000)) implica que as excitações colisionais para cada nível dependam da energia que possuem os elétrons livres. A ocupação do nível ${ }^{1} S_{0}$ depende das partículas em colisão terem uma temperatura eletrônica suficientemente alta para excitar este nível. Se não, somente levarão o íon ao estado ${ }^{1} D_{2}$. 

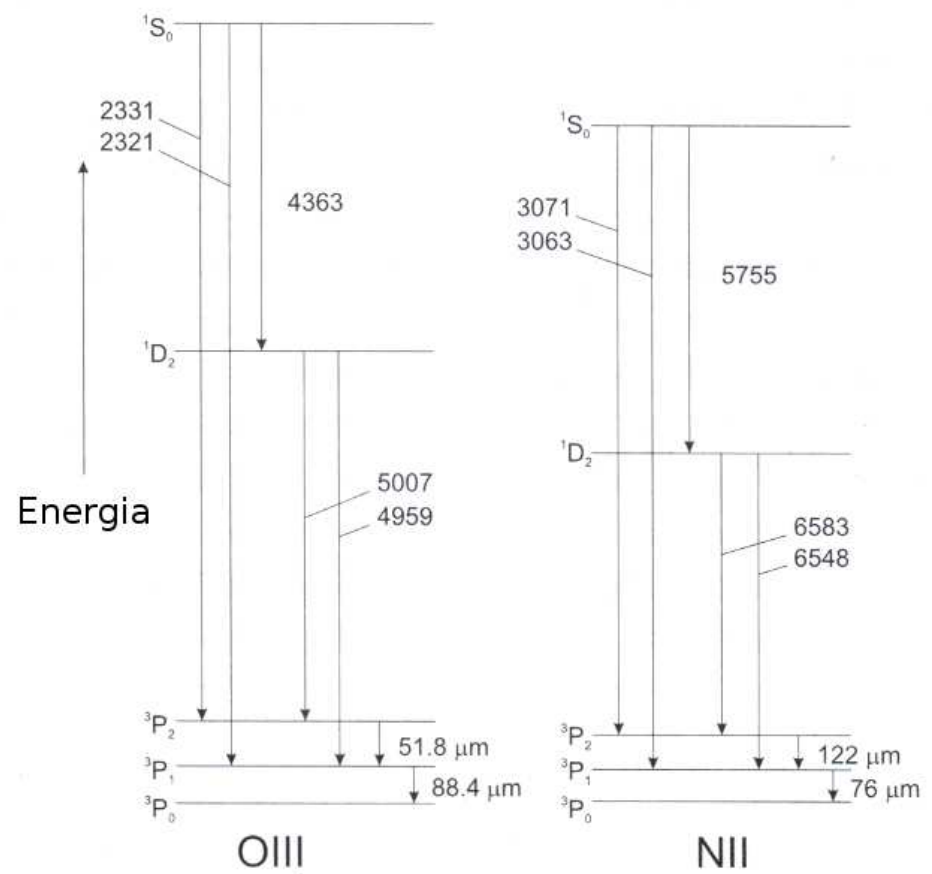

Figura 2.2: Os diagramas de energia de [OIII] e [NII]. Por padrão, os comprimentos de onda das transições são indicados em Å.

A quantidade de elétrons capazes de promover a excitação, seja ao nível ${ }^{1} S_{0}$ ou ${ }^{1} D_{2}$, se refletirá no número de fótons emitidos por cada um destes níveis. As intensidades das linhas provenientes de um estado excitado estão relacionados pelos coeficientes de Einstein. Quando comparamos a razão da intensidade das linhas de emissão de um mesmo íon, estamos comparando quantos fótons estão no nível superior em relação ao nível inferior.

Num átomo de três níveis, o equilíbrio estatístico se dará pelas equações:

$$
\begin{aligned}
n_{2} A_{21} & =n_{1} n_{e} C_{12} \\
n_{3}\left(A_{31}+A_{32}\right) & =n_{1} n_{e} C_{13}
\end{aligned}
$$

onde os níveis 1, 2 e 3 correspondem aos estados $\mathrm{P}, \mathrm{D}$ e $\mathrm{S}$, respectivamente. A razão $n_{2} / n_{3}$ pelas equações 2.15 e 2.16 será:

$$
\frac{n_{2}}{n_{3}}=\frac{C_{12}}{C_{13}} \frac{A_{31}+A_{32}}{A_{21}}
$$


Para o caso do $\mathrm{O}^{++}$, a intensidade da razão de linhas $\lambda 5007+\lambda 4959$ sobre $\lambda 4363$ será:

$$
\begin{aligned}
\frac{I(\lambda 5007+4959)}{I(\lambda 4363)} & =\frac{n_{2} \nu_{21} A_{21}}{n_{3} \nu_{32} A_{32}} \\
& =\frac{C_{12}}{C_{13}}\left(\frac{A_{31}+A_{32}}{A_{32}}\right) \frac{\nu_{21}}{\nu_{32}} .
\end{aligned}
$$

Onde $A_{j i}$ são os coeficientes de emissão espontânea determinados a partir de estudos de estrutura atômicos e $C_{j i}=C_{j i}\left(T_{e}\right)$ o coeficiente de excitação colisional como função da temperatura eletrônica $T_{e}$.

\subsubsection{Abundâncias iônicas}

A abundância relativa de íons presentes nas NPs pode ser calculada a partir da intensidade de linhas proibidas emitidas comparadas com o fluxo emitido em $\mathrm{H} \beta$.

Tais intensidades devem levar em conta as propriedade atômicas de cada íon, e são fortemente dependentes da temperatura e a densidade eletrônica da região emissora.

Neste trabalho, as abundâncias iônicas relativas são calculadas utilizando os valores locais de densidade e temperatura eletrônicas definidos previamente no objeto, sendo calculados com a rotina IONIC (pacote STDSAS/NEBULAR) dentro do ambiente IRAF. Para alguns íons, há mais de uma transição observada no visível. Neste caso, a abundância foi escolhida como a média entre os valores obtidos, ponderados pela intensidade das linhas mais brilhantes.

É interessante registrar o papel das duas temperaturas eletrônicas consideradas, uma derivada a partir das linhas de [NII] e outra das linhas de [OIII]. Estas linhas refletem duas condições físicas diferentes. Assim, para os elementos uma vez ionizados $\left(\mathrm{N}^{+}, \mathrm{O}^{+}, \mathrm{e}\right.$ $\mathrm{S}^{+}$) a temperatura considerada para o cálculo é o das linhas [NII], enquanto que para os outros íons, utiliza-se a temperatura das linhas do [OIII]. Lembrando que, devido ao seu alto potencial de ionização, o cálculo da abundância de $\mathrm{HeI}\left(\mathrm{He}^{+}\right)$utiliza a temperatura das linhas [OIII].

As abundâncias iônicas do Hélio são calculadas a partir da teoria de recombinação para este elemento, como correção da desexcitação colisional. O elemento He também desempenha um importante papel para as abundâncias. Uma vez que neste elemento a estrutura eletrônica é bem conhecida, as abundâncias iônicas do He (HeI e HeII) são obtidas pela teoria de recombinação. Destacamos que somente para este elemento, a notação de 
ionização é diferenciada $\left(\mathrm{HeI} \equiv \mathrm{He}^{+}\right.$e $\left.\mathrm{HeII} \equiv \mathrm{He}^{++}\right)$.

Utilizamos as seguintes expressões para as abundâncias iônicas do Hélio, com $t$ sendo a temperatura em unidades de $10^{4} \mathrm{~K}$ e $f_{\lambda}$ o fluxo no comprimento de onda $\lambda$ dados em $\AA$. Os coeficientes de recombinação utilizados no trabalho são os apresentados por Pequignot et al. (1991), com correção de desexcitação colisional para o He I de Kingdon e Ferland (1995):

$$
\begin{gathered}
a=\frac{0,668 t^{-0,507}}{1+1,221 t^{0,653}} \\
b_{4471}=\frac{4471}{4861}\left(\frac{f_{4471}}{f_{H \beta}}\right) \frac{1+1,505 t^{0,779}}{0,79 \times 0,429 t^{-0,53}} \\
c_{4471}=\frac{6,95 t^{0,15} e^{-4,545 / t}+0,22 t^{-0,55} e^{-4,884 / t}+0,98 t^{-0,45} e^{-4,901 / t}}{1+3130 t^{-0,5} d^{-1}} \\
c_{5876}=\frac{6,78 t^{0,07} e^{-3,776 / t}+1,67 t^{-0,15} e^{-4,545 / t}+0,60 t^{-0,34} e^{-4,901 / t}}{1+3130 t^{-0,5} d^{-1}}=\frac{5876}{4861}\left(\frac{f_{5876}}{f_{H \beta}}\right) \frac{1+1,683 t^{0,667}}{1,323 t^{-0,696}} \\
\frac{\left[\frac{H e^{+}}{H}\right]_{\lambda}=\frac{a \times b_{\lambda}}{1+c_{\lambda}}}{H}=a \times 0,5 \frac{4686}{4861}\left(\frac{f_{4686}}{f_{H \beta}}\right) \frac{1+2,884 \times(t / 4)^{0,609}}{1,549 \times(t / 4)^{-0,693}}
\end{gathered}
$$

\subsubsection{Abundâncias químicas}

A fim de converter os fluxos de linha mensuradas em abundâncias químicas de NPs, Torres-Peimbert e Peimbert (1979) desenvolvem o método dos fatores de correção de ionização (FCIs, ou ICFs em inglês). Neste método, as abundâncias químicas elementais são determinadas a partir das abundâncias iônicas observadas, multiplicadas por seu respectivo FCI. Estes fatores expressam a contribuição à abundância de íons não observados.

As expressões permitem obter as abundâncias totais relativas ao Hidrogênio quando somente linhas no óptico são mensuradas. As expressões 2.26 à 2.30 são de Kingsburgh e 
Barlow (1994), enquanto que a expressão 2.31 é de de Freitas-Pacheco et al. (1993). Na notação utilizada, $\frac{X}{X^{n+}} \equiv \frac{X / H}{X^{n+} / H}$.

$$
\begin{gathered}
\frac{H e}{H}=\frac{H e^{+}}{H^{+}}+\frac{H e^{++}}{H^{+}} \\
\frac{O}{H}=\left(\frac{O^{+}}{H^{+}}+\frac{O^{++}}{H^{+}}\right) \frac{H e}{H e^{+}} \\
\frac{N}{H}=\frac{N^{+}}{H^{+}} \frac{O}{O^{+}} \\
\frac{S}{H}=\left(\frac{S^{+}}{H^{+}}+\frac{S^{++}}{H^{+}}\right)\left[1-\left(1-\frac{O^{+}}{O}\right)^{3}\right]^{-1 / 3} \\
\frac{N e}{H}=\frac{N e^{++}}{H^{+}} \frac{O}{O^{++}} \\
\frac{A r}{H}=1.34\left(\frac{A r^{++}}{H^{+}} \frac{O}{O^{++}}\right)
\end{gathered}
$$

\subsubsection{Diagramas de diagnóstico do plasma}

A razão de fluxo de linhas proibidas, combinada ou não com o fluxo de linhas Hidrogênio, também pode ser utilizada como ferramenta para diagnóstico do plasma nebular. Sabbadin et al. (1977) classifica um objeto a partir de mapas de regiões onde razões de linhas de Nebulosas Planetárias são emitidas em comparação com regiões de propriedades distintas, como remanescentes de supernovas e regiões HII.

Destacamos as seguintes razões de linhas utilizadas nestes trabalho:

- $\mathrm{H} \alpha /[\mathrm{NII}](\lambda 6548+6584)$, razão que reflete um diagnóstico da densidade/temperatura do gás, comparando a emissividade de um dado íon com a recombinação do Hidrogênio. A razão $\mathrm{H} \alpha /[\mathrm{SII}](\lambda 6725)$ pode ser utilizada de maneira análoga.

- $[\operatorname{SII}](\lambda 6716 / \lambda 6731)$, indicativo da densidade eletrônica, uma vez que a temperatura local tem efeitos secundários.

- $\log [\mathrm{SIII}](\lambda 6312) /[\mathrm{SII}](\lambda 6725)$, indicativo das regiões de diferentes potenciais de ionização presentes na nuvem. Devido à escala logarítmica, o contraste entre determinadas regiões será evidenciada se observado o logaritmo do inverso da razão de linhas. Assim, neste trabalho serão reportados os resultados da razão de linhas e de seu inverso na escala logarítmica. 
Estes tipos de diagramas são úteis na investigação de regiões com razões de linhas anômalas. Um exemplo de utilização destes diagramas está em Phillips e Cuesta (1999), que os utilizaram para comparar a emissão anômala de M2-9 com regiões excitadas por choques.

\subsubsection{Erros estimados e incertezas envolvidas}

Uma das principais fontes de erros no processo de cálculo das abundâncias está na obtenção dos fluxos das linhas de emissão observadas. Este erro é intrínseco ao processo de obtenção nos parâmetros físicos nebulares (densidades e temperaturas) e é propagado na determinação de abundâncias iônicas e consequentemente para a determinação de abundâncias elementais.

A fim de estimar os erros na determinação destas grandezas, foram efetuadas simulações do tipo Monte Carlo a partir das incertezas esperadas para o fluxo das linhas espectrais. Um número de espectros sintéticos é construído (250 neste trabalho), onde o fluxo de cada linha é variado aleatoriamente dentro de um perfil gaussiano proporcional ao seu intervalo de erro.

Os intervalos de erro são estimados a partir da relação entre os erros nos fluxos observados e aqueles obtidos de um ajuste linear com a média registrada dos fluxos. As abundâncias e parâmetros físicos finais são determinados pela média dos valores derivados de cada espectro sintético, com o erro associado com o desvio padrão do conjunto.

O erro estimado desta forma leva em conta somente a influência das incertezas do fluxo das linhas. Para estimativas de outras fontes de erros (como a associada aos fatores de correção de ionização nas abundâncias químicas), veja a seção 3.3. 
Capítulo 3

\section{Simulações}

\subsection{Cloudy e Cloudy_3D}

Cloudy é um código de fotoionização unidimensional, cuja descrição é feita por Ferland et al. (1998). Assim, só pode ser diretamente aplicado em nebulosas com simetria esférica. Para correta modelagem de objetos não esféricos, alternativamente a códigos tridimensionais que são de execução complicada e lentos computacionalmente, é possível utilizar diagramas tridimensionais montados a partir de iterações unidimensionais.

A desvantagem de simulações por iterações unidimensionais é a ausência da transmissão de radiação para zonas não radialmente alinhadas. Isto pode ser importante, por exemplo, se houver zonas de maior densidade do material, que radialmente causariam uma "sombra" na transmissão, região onde poderia ser atingida por esta componente não radial. Entretanto, isto não deve ser um caso recorrente para NPs, que devido às suas baixas opacidades, o campo de radiação radial é o que predomina.

$\mathrm{Na}$ literatura são reportados alguns códigos para este fim. Um exemplo de código tridimensional é o Mocassin (Ercolano et al., 2008). Neste trabalho foi utilizado o código Cloudy_3D, descrito por Morisset (2006), que se utiliza do Cloudy para as iterações unidimensionais.

Parâmetros mínimos para simulação no Cloudy:

- Luminosidade da fonte central

- Espectro da fonte central

- Composição química da nuvem, com informação sobre a presença de grãos 
- Densidade de H para a nuvem

- Distância da nuvem à fonte central

- Informações básicas sobre a geometria (esférica, plano-paralela, etc.)

A densidade de hidrogênio e composição química são obtidos diretamente dos espectros, para cada região analisada. As outras informações da relação acima devem ser pesquisadas na literatura para a simulação dos objetos. As temperaturas eletrônicas são consequências do campo de radiação da nuvem definida, e podem funcionar como um vínculo na simulação.

Para a execução do Cloudy_3D, é ainda necessária a função que descreve a distribuição volumétrica de matéria na NP. O projeto destina-se à simulação de NPs bipolares por questões de simetria: ao longo do eixo principal, as mesmas são idênticas em relação à distância ao centro, e exibem simetria azimutal em torno deste eixo. Desta forma, se o volume da simulação for dividido num cubo de 8 "quadrantes", as iterações só são necessárias em um deles; nos 7 demais cubos, projetam-se os fluxos simulados do cubo inicial.

Por fim, as comparações com os dados observacionais são feitas a partir da definição do ângulo $i$ de inclinação do objeto em relação ao observador e da projeção da estrutura tridimensional simulada no plano do céu, onde se pode comparar a imagem do objeto e os espectros em aberturas análogas às das observações.

O programa Cloudy faz algumas aproximações para contornar as restrições de campo unicamente radial. Por exemplo, a radiação contínua que escaparia da nuvem na direção da fonte central deve interagir com o gás presente do outro lado da fonte. Esta é a definição de uma geometria "fechada" para o programa.

Estas interações são acionadas pelo comando sphere. Esta opção é recomendada sempre que a geometria é esférica ou o gás cobre quase totalmente a fonte central, e foi sempre acionada para as simulações deste trabalho. Na maioria dos casos, a documentação do programa informa que esta opção trará uma diferença de 10 a 15\% nas quantidades previstas.

Um exemplo de aplicação do código Cloudy_3D, com detalhes das simulações, é reportado por Zepeda (2006). 


\subsection{Parâmetros e vínculos das simulações}

A qualidade de um modelo é proporcional à quantidade de vínculos que ele é capaz de reproduzir. Desta forma, enumeramos os vínculos utilizados nas simulações para o confronto com os dados observacionais e de literatura com as simulações. Como vínculos diretos, temos:

- Tamanhos angulares espectroscópicos

- Fluxo $\mathrm{H} \beta$ total

- Perfil de densidade [SII]

- Variação angular dos parâmetros ou dos fluxos

O tamanho angular é definido como "espectroscópico", pois dependerá do comprimento de onda observado. Tal efeito é bem conhecido para o caso das NPs. Este tamanho corresponde também ao tamanho da região brilhante, que é simulada. Sabe-se que as dimensões podem variar bastante, principalmente se consideradas as emissões de baixo brilho superficial, como os halos. Um estudo observacional de um conjunto de halos é feito por Corradi et al. (2003).

Como vínculos indiretos nas observações, temos:

- Propriedades da fonte central (Luminosidade e Temperatura)

- Parâmetros físicos e composição química médios

- Distância

- Massa ionizada

Os vínculos indiretos são os parâmetros de entrada (tais como a fonte central e composição química) ou grandezas que resultam diretamente das simulações (temperaturas eletrônicas, distância, massa ionizada). A utilização destes parâmetros e inclusão nos modelos finais são discutidos nas próximas seções.

Para a definição do melhor modelo buscou-se a reprodução mais próxima dos vínculos diretos (variações menores que 10\%), exceto para a variação angular dos parâmetros. Neste 
caso, escolheu-se o modelo que apresentasse o melhor valor de $\chi^{2}$ para avaliar as variações experimentais comparadas com as previsões dos modelos. $\chi^{2}$ é definido:

$$
\chi^{2}=\sum_{i=1}^{N} \frac{\left(y_{i}-f\left(x_{i}\right)\right)^{2}}{\sigma_{i}^{2}},
$$

onde $y_{i}$ é um dado experimental distribuído em torno do ajuste (ou modelo) $f\left(x_{i}\right)$, com incerteza $\sigma_{i}$. $N$ é o número de parâmetros de um dado objeto, determinados para cada abertura $i$ do objeto. A abertura $i$ sob fenda-longa será a janela de extração, enquanto sob campo integral será o elemento de resolução da unidade. Desta forma, pode-se quantificar as diferenças entre as previsões do modelo e os dados experimentais, ponderados pela incerteza em cada ponto.

Para uma descrição de $\chi^{2}$ no contexto de utilização como teste de significância em dados experimentais, consultar Helene e Vanin (1981).

\subsubsection{Fonte central}

Existem diferentes métodos para estimar a temperatura efetiva das estrelas centrais de NPs (ECNPs, ou CSPNs). Dentre os mais utilizados, estão a Temperatura de Zanstra para H e He (método de recombinação); Temperatura de Stoy; Temperatura de Ambartsumyan (método HeII/HI). Uma revisão dos principais métodos está presente em Gurzadyan (1997).

Os diferentes métodos podem levar as estimativas não compatíveis entre si, em função das hipóteses consideradas. Por exemplo, no método de Zanstra assume-se que a planetária é opticamente espessa para o contínuo de Lyman do Hidrogênio. Se esta condição não for verdadeira, a estimativa da temperatura é um limite inferior. A temperatura efetiva determinada nos modelos é comparada com valores de referência, apontados em cada caso.

As ECNPs podem ser divididas em dois grupos básicos, em função de seu espectro: as ricas em Hidrogênio em oposição às pobres em Hidrogênio. Para esta diferenciação foi consultado o catálogo de Acker et al. (1992). Na ausência de informação para o espectro de uma ECNP neste catálogo, outra referência foi indicada. Com base nos espectros sintéticos de ECNPs desenvolvido por Rauch (2003), utilizamos o conjunto denominado por $H-N i^{1}$ para as planetárias ricas em $\mathrm{H}$, e para as H-pobres o conjunto $P G 1159^{1}$ (também

\footnotetext{
${ }^{1}$ São mantidas as denominações destes tipos espectrais feitas originalmente pelo autor.
} 
chamado de $\mathrm{He}+\mathrm{C}+\mathrm{N}+\mathrm{O}$ ). Neste último conjunto, são incluídas as planetárias com núcleo classificado como Wolf-Rayet ${ }^{2}$. O espectro de linhas de emissão destas estrelas origina-se inteiramente no vento estelar, que depende não só da temperatura e luminosidade da estrela central, mas também da taxa de perda de massa, velocidade do vento, entre outros fatores (Gray e Corbally, 2009).

No caso da indisponibilidade de informações espectrais da fonte central, utilizamos o conjunto $H$-Ni, uma vez que a razão entre núcleos ricos em H e pobres é de $\sim 4 / 1$ (Kwok, 2000).

Salientamos que, uma vez que ECNPs têm uma ampla variedade de tipos espectrais e abundâncias fotosféricas, a hipótese de corpo negro provavelmente não é pior do que um conjunto específico de modelos atmosféricos, como pode ser observado em Kaler (1983).

\subsubsection{Geometria e perfil de densidade}

Para uma adequada compreensão da morfologia das NPs, as estruturas bidimensionais vistas em suas imagens necessitam ser associadas a estruturas tridimensionais. Com base no cenário de ventos interagentes, duas estruturas básicas foram utilizadas nas simulações: i) elipsóide prolato ou esfera; ii) estrutura bipolar em forma de "ampulheta" ou tubo.

No código Cloudy_3D, esta estrutura é definida através de uma função que associa a densidade para cada ponto do espaço, a partir de um centro geométrico (função de raio e $\theta$ ). Para cada objeto, indicamos a função utilizada. Em geral, a densidade é considerada nula até um raio mínimo denominado de raio interno, a partir do qual a estrutura é descrita. Esta hipótese é confirmada por simulações hidrodinâmicas da evolução das NPs, como descrito por Schonberner (1997).

Destacamos que a descrição do perfil de densidade pode ser feita de distintas formas. Por exemplo, Kahn e West (1985) adotam o seguinte perfil de densidade para as nebulosas planetárias:

$$
n(\theta)=n(0)\left(1+\epsilon \cos ^{m} \theta\right)
$$

onde $\mathrm{n}(0)$ é a densidade no equador $(\theta=0),(1+\epsilon)$ é a razão entre densidades no equador sobre o pólo, e $m$ é o parâmetro que determina a mudança no gradiente entre o equador

\footnotetext{
${ }^{2}$ Será utilizada também a sigla wel, de "weak emission lines" em inglês, para o tipo espectral com fracas linhas em emissão.
} 
para o pólo.

\subsubsection{Composição química}

Assume-se composição química homogênea para as simulações. Os valores utilizados são os obtidos através dos fluxos médios, por apresentar menor incerteza. Seus valores são comparados com dados de literatura, e mantidos para as simulações (salvo quando indicado o contrário).

A análise das linhas mais brilhantes do espectro visível permite a estimativa da abundância de seis elementos em relação ao Hidrogênio: Hélio, Oxigênio, Nitrogênio, Enxofre, Neônio e Argônio (He, O, N, S, Ne, Ar). O código Cloudy é capaz de utilizar todos os elementos químicos até $\mathrm{Z}=30$. Para as abundâncias dos elementos químicos não determinados observacionalmente, utilizou-se os valores de Khromov (1989) para as NPs Galácticas, com alto esgotamento para os elementos que não são mencionados.

\subsection{Erros estimados e incertezas envolvidas}

Alexander e Balick (1997) investigam a acurácia da utilização dos FCIs através de simulações com o código de fotoionização Cloudy para as condições típicas de nebulosas planetárias. De acordo com este trabalho, a utilização de FCIs para determinação de abundâncias médias gera valores com discrepância da ordem de 20\%. Para NPs angularmente resolvidas, os erros associados aos FCIs podem ser localmente altos, especialmente para N, Ne e Ar, em regiões com considerável abundância de elementos neutros. A magnitude dos erros no método de FCIs difere em NPs de acordo com seus tamanhos e fontes de ionização. Além disso, os erros dependem do grau em que os fótons ionizantes são absorvidos ou escapam (ou seja, se a nebulosa é limitada por ionização ou por densidade). Em NPs cuja estrela central tenha temperatura efetiva menor que 45.000K, há uma maior discrepância nas abundâncias determinadas pelo método de FCIs. 
Capítulo 4

\section{Resultados}

\subsection{Espectroscopia de fenda longa}

\subsubsection{IC4406}

IC4406 é uma típica NP que apresenta simetria bipolar. Na tabela 4.1 estão os valores dos parâmetros físicos médios e da composição química, obtidos pela integração dos fluxos em toda a fenda sobre objeto. A orientação da fenda do céu está indicada no quadro direito da figura 4.1. Os valores da composição química obtidos foram os utilizados nas simulações. Os fluxos obtidos e as incertezas correspondentes estão no apêndice C.1.

Tabela 4.1 - Parâmetros físicos e composição química obtidos com integração por todo o objeto IC4406.

\begin{tabular}{lrr|lll}
\hline \hline Parâmetro & Valor & $\sigma$ & Parâmetro & Valor & $\sigma$ \\
\hline $\bar{n}_{e}$ & 771 & 692 & $\mathrm{He}^{+} / \mathrm{H}$ & 0,13 & 0,02 \\
$\bar{T}_{[N I I]}$ & 9989 & 815 & $\mathrm{He}^{++} / \mathrm{H}$ & 0,003 & 0,001 \\
$\bar{T}_{[\text {OIII }]}$ & 10254 & 656 & $\mathrm{O}^{+} / \mathrm{H}$ & $2,0 \mathrm{e}-04$ & $7,9 \mathrm{e}-05$ \\
$\mathrm{He} / \mathrm{H}$ & 0,13 & 0,02 & $\mathrm{O}^{++} / \mathrm{H}$ & $3,3 \mathrm{e}-04$ & $7,6 \mathrm{e}-05$ \\
$\epsilon(\mathrm{O})$ & 8,74 & 0,09 & $\mathrm{~N}^{+} / \mathrm{H}$ & $8,6 \mathrm{e}-05$ & $2,0 \mathrm{e}-05$ \\
$\epsilon(\mathrm{N})$ & 8,37 & 0,07 & $\mathrm{~S}^{+} / \mathrm{H}$ & $6,6 \mathrm{e}-07$ & $1,7 \mathrm{e}-07$ \\
$\epsilon(\mathrm{S})$ & 6,31 & 0,15 & $\mathrm{~S}^{++} / \mathrm{H}$ & $1,2 \mathrm{e}-06$ & $5,8 \mathrm{e}-07$ \\
$\epsilon(\mathrm{Ne})$ & 8,51 & 0,11 & $\mathrm{Ne}^{3+} / \mathrm{H}$ & $2,0 \mathrm{e}-04$ & $5,4 \mathrm{e}-05$ \\
$\epsilon(\mathrm{Ar})$ & 6,75 & 0,09 & $\mathrm{Ar}^{3+} / \mathrm{H}$ & $2,5 \mathrm{e}-06$ & $4,7 \mathrm{e}-07$ \\
\hline
\end{tabular}



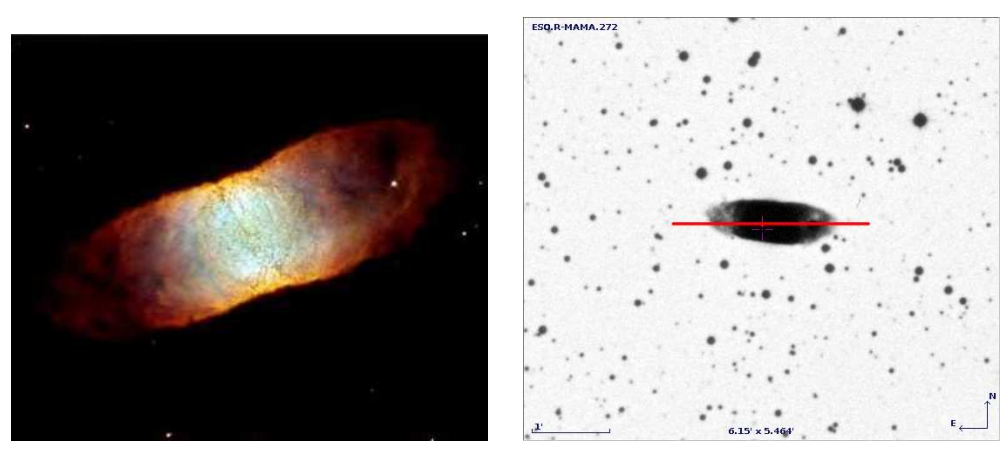

Figura 4.1: Imageamento de IC4406 feito pelo telescópio espacial Hubble (cores R:G:B = $[\mathrm{NII}]: \mathrm{H} \alpha:[\mathrm{OIII}]$, à esquerda) e indicação do posicionamento da fenda em imagem do catálogo ESO.R-MAMA (à direita).

O diâmetro obtido na espectroscopia foi de 111"para este objeto. A partir do imageamento do objeto (fig.4.1), a razão entre eixos determinada é de $\sim 0,27$. Tal razão, extremamente baixa, é um indicativo de que o objeto está alinhado com o plano do céu (perpendicular à linha de visada, $i=90^{\circ}$ ). Este hipótese foi mantida nas simulações.

Visto de perfil, este objeto não tem indícios da concavidade presente em NPs bipolares de geometria "ampulheta". Assim, no cenário de morfologia de ventos interagentes, este objeto pode ser enquadrado num estado intermediário entre elipsóide e "ampulheta". Geralmente nos objetos bipolares há uma sobredensidade na região central em relação às suas extremidades. Gruenwald et al. (1997) descrevem esta característica neste objeto como um toro mais denso que o material bipolar.

O modelo deste trabalho aproxima a geometria de IC4406 a um tubo. Para a descrição da sobredensidade, é utilizada uma função proporcional a determinada por Dayal et al. (2000) para MyCn18, vinculando o fator pelas densidades obtidas ao longo da fenda.

Dado o grande tamanho angular de IC4406, foram determinadas as propriedades físicas e respectivas abundâncias químicas em 13 aberturas. Os parâmetros determinados estão na tabela 4.2, que inclui também os valores de $\chi^{2}$ em comparação do modelo para cada dos parâmetros. As janelas listadas na tabela foram escolhidas sobre a fenda indicada na figura 4.1, em ordem crescente de leste para oeste. Na tabela C.9 são exibidos os fluxos determinados para cada uma das janelas de extração, comparados com os fluxos previstos pelo modelo. 
Como pode ser visto na figura 4.2, é marcante a maior densidade eletrônica da região central em relação às extremidades, com valores de temperaturas eletrônicas muito próximas para as linhas de [OIII] e [NII]. A estrutura de ionização da nuvem também é bem determinada, como visto no comportamento das abundâncias iônicas dos Hélio e Oxigênio, uma e duas vezes ionizados.

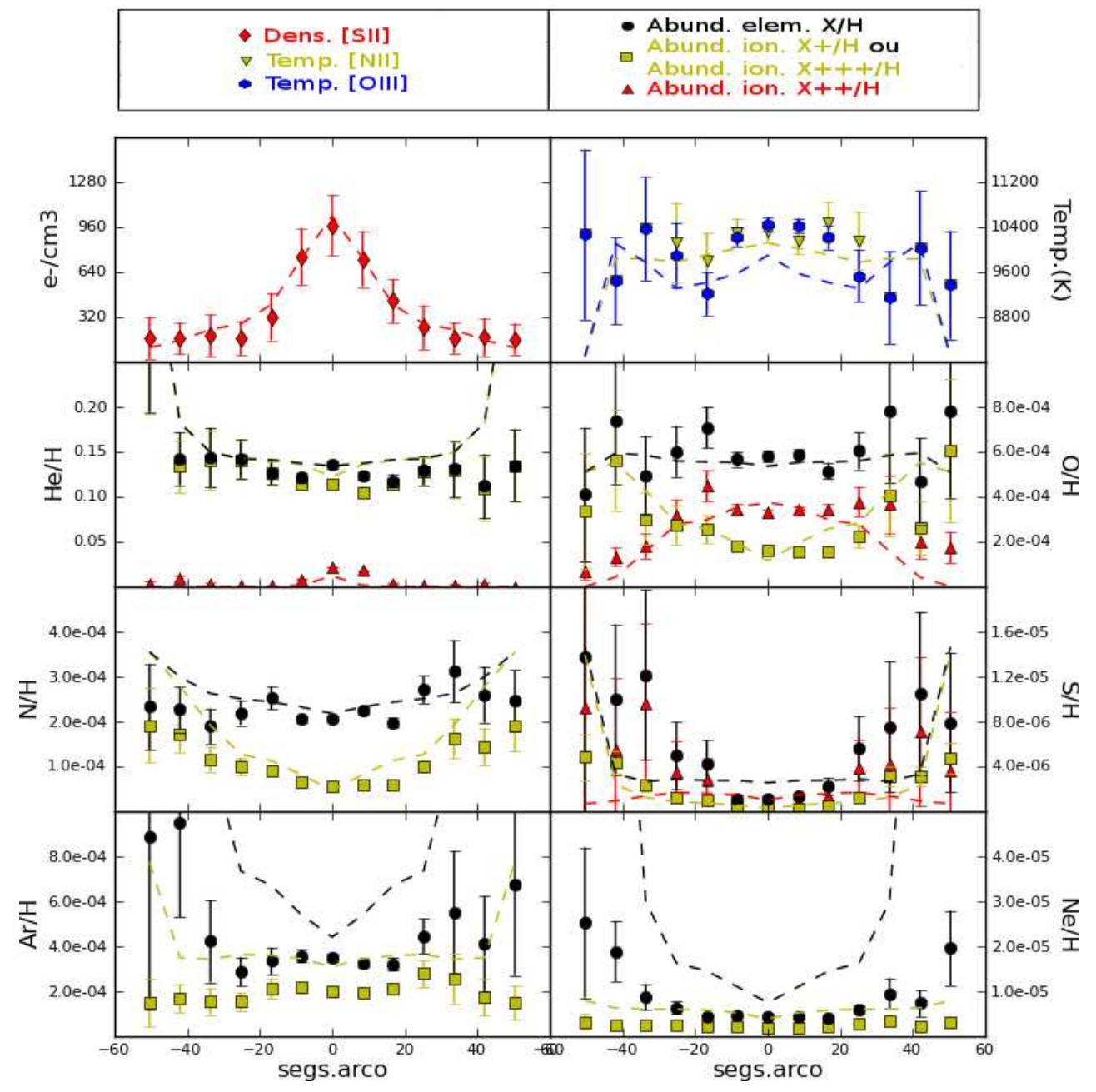

Figura 4.2: Parâmetros físicos e composição química obtidos para IC4406 (pontos) e respectiva previsão do modelo (linha tracejada).

As variações nas abundâncias não são mudanças reais na composição química do material, mas antes uma tendência intrínseca do método de FCIs. Esta tendência é mantida quando computadas as abundâncias com os fluxos esperados em cada uma das janelas a partir do modelo de composição homogênea.

Os parâmetros finais do modelo são apresentados na tabela 4.3, e respectiva comparação 
com dados de literatura. O avermelhamento registrado em cada região das fendas está na figura 4.3.

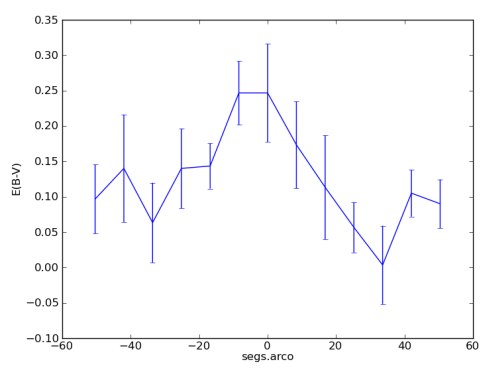

Figura 4.3: Variação do parâmetro de avermelhamento E(B-V) ao longo da fenda de IC4406. 
Tabela 4.2 - Parâmetros físicos e composição química previstos pelo modelo de IC4406, e respectivo valor de $\chi^{2}$ em relação às observações. Dada a simetria do modelo, são exibidos somente os valores até a janela central.

\begin{tabular}{|c|c|c|c|c|c|c|c|c|}
\hline Parâmetro & $\chi^{2}$ & Janela 1 & Janela 2 & Janela 3 & Janela 4 & Janela 5 & Janela 6 & Janela 7 \\
\hline $\mathrm{n}_{e}$ & 5,0 & 100 & 180 & 253 & 287 & 447 & 789 & 1000 \\
\hline $\mathrm{T}_{[N I I]}$ & 6,8 & 8776 & 10068 & 10003 & 9998 & 10126 & 10316 & 10323 \\
\hline $\mathrm{T}_{[O I I I]}$ & 20,9 & 8776 & 10119 & 9813 & 9478 & 9632 & 9756 & 9958 \\
\hline $\mathrm{He}^{+} / \mathrm{H}$ & 25,0 & 0,349 & 0,161 & 0,144 & 0,139 & 0,139 & 0,134 & 0,123 \\
\hline $\mathrm{He}^{++} / \mathrm{H}$ & 68,6 & 0,000 & 0,000 & 0,000 & 0,000 & 0,000 & 0,001 & 0,012 \\
\hline $\mathrm{He} / \mathrm{H}$ & 17,8 & 0,349 & 0,161 & 0,144 & 0,139 & 0,139 & 0,136 & 0,135 \\
\hline $\mathrm{O}^{+} / \mathrm{H}$ & 15,6 & $5,20 \mathrm{E}-4$ & $4,90 \mathrm{E}-4$ & $3,10 \mathrm{E}-4$ & $2,10 \mathrm{E}-4$ & $1,90 \mathrm{E}-4$ & $1,40 \mathrm{E}-4$ & $8,50 \mathrm{E}-5$ \\
\hline $\mathrm{O}^{++} / \mathrm{H}$ & 21,1 & $7,30 \mathrm{E}-7$ & $1,10 \mathrm{E}-4$ & $2,50 \mathrm{E}-4$ & $3,30 \mathrm{E}-4$ & $3,50 \mathrm{E}-4$ & $3,90 \mathrm{E}-4$ & $3,90 \mathrm{E}-4$ \\
\hline $\mathrm{fci}_{O}$ & - & 1,00 & 1,00 & 1,00 & 1,00 & 1,00 & 1,01 & 1,10 \\
\hline$\epsilon(\mathrm{O})$ & 13,2 & 8,71 & 8,78 & 8,76 & 8,74 & 8,73 & 8,73 & 8,72 \\
\hline $\mathrm{N}^{+} / \mathrm{H}$ & 26,3 & $3,80 \mathrm{E}-4$ & $2,30 \mathrm{E}-4$ & $1,40 \mathrm{E}-4$ & $9,40 \mathrm{E}-5$ & $8,40 \mathrm{E}-5$ & $5,80 \mathrm{E}-5$ & $3,50 \mathrm{E}-5$ \\
\hline $\mathrm{fci}_{N}$ & - & 1,00 & 1,22 & 1,81 & 2,56 & 2,80 & 3,86 & 6,19 \\
\hline$\epsilon(\mathrm{N})$ & 15,6 & 8,58 & 8,45 & 8,40 & 8,38 & 8,37 & 8,35 & 8,33 \\
\hline $\mathrm{S}^{+} / \mathrm{H}$ & 29,2 & $9,00 \mathrm{E}-6$ & $1,70 \mathrm{E}-6$ & $8,80 \mathrm{E}-7$ & $6,30 \mathrm{E}-7$ & $5,40 \mathrm{E}-7$ & $3,80 \mathrm{E}-7$ & $2,40 \mathrm{E}-7$ \\
\hline $\mathrm{S}^{++} / \mathrm{H}$ & 12,3 & $4,50 \mathrm{E}-7$ & $1,20 \mathrm{E}-6$ & $1,60 \mathrm{E}-6$ & $1,50 \mathrm{E}-6$ & $1,40 \mathrm{E}-6$ & 1,30E-6 & $9,80 \mathrm{E}-7$ \\
\hline $\operatorname{fci}_{S}$ & - & 1,00 & 1,01 & 1,10 & 1,29 & 1,36 & 1,69 & 2,43 \\
\hline$\epsilon(\mathrm{S})$ & 21,4 & 6,98 & 6,45 & 6,43 & 6,45 & 6,43 & 6,47 & 6,47 \\
\hline $\mathrm{Ne}^{3+} / \mathrm{H}$ & 71,5 & $7,10 \mathrm{E}-4$ & $3,60 \mathrm{E}-4$ & $3,50 \mathrm{E}-4$ & $3,50 \mathrm{E}-4$ & $3,40 \mathrm{E}-4$ & $3,40 \mathrm{E}-4$ & $3,10 \mathrm{E}-4$ \\
\hline $\mathrm{fci}_{N e}$ & - & 713 & 5,50 & 2,24 & 1,64 & 1,56 & 1,37 & 1,34 \\
\hline$\epsilon(\mathrm{Ne})$ & 50,8 & 11,70 & 9,29 & 8,89 & 8,77 & 8,73 & 8,66 & 8,61 \\
\hline $\mathrm{Ar}^{3+} / \mathrm{H}$ & 140,6 & 8,80E-6 & $6,20 \mathrm{E}-6$ & 5,90E-6 & $5,70 \mathrm{E}-6$ & $5,40 \mathrm{E}-6$ & 4,80E-6 & $3,50 \mathrm{E}-6$ \\
\hline $\mathrm{fci}_{A r}$ & - & 955 & 7,37 & 3,00 & 2,20 & 2,09 & 1,83 & 1,79 \\
\hline$\epsilon(\mathrm{Ar})$ & 88,7 & 9,92 & 7,66 & 7,25 & 7,10 & 7,05 & 6,94 & 6,80 \\
\hline $\mathrm{E}(\mathrm{B}-\mathrm{V})$ & - & 0,10 & 0,14 & 0,06 & 0,14 & 0,14 & 0,25 & 0,25 \\
\hline
\end{tabular}


Tabela 4.3 - Parâmetros do modelo final de IC4406 comparados com referências.

\begin{tabular}{cccc}
\hline \hline Parâmetro & Modelo & Valores de Ref. & Referências \\
\hline $10^{3} . T_{\text {eff }}(\mathrm{K})$ & 105 & $94 ; 125,9 ; 80$ & KJ89; M97; G97 \\
$\mathrm{L} / \mathrm{L}_{\odot}$ & 160 & $640 ; 110 ; 400$ & KJ89; M97; G97 \\
Espectro & PG1159 & WR & A92 \\
$\log \mathrm{g}\left(\mathrm{cm} / \mathrm{s}^{2}\right)$ & 7,6 & 7,56 & M97 \\
$\bar{E}(B-V)$ & 0,35 & 0,$26 ; 0,33 ; 0,4$ & W83; KJ89; T92 \\
$\mathrm{F}_{H \beta}^{\text {total }}\left(\mathrm{erg} / \mathrm{cm}^{2} \mathrm{~s}\right)$ & $4,02 \mathrm{E}-011$ & $4,07 \mathrm{E}-011$ & $\mathrm{~A} 92$ \\
$\mathrm{~d}(\mathrm{kpc})$ & 1,11 & 0,92 & $\mathrm{M} 97$ \\
$\mathrm{M}_{\text {ion }} / \mathrm{M}_{\odot}$ & 0,08 & - & - \\
$i(\mathrm{grau})$ & $90^{\circ}$ & - & - \\
\hline
\end{tabular}

KJ89:Kaler e Jacoby (1989); M97:Mal'Kov (1997); G97:Gruenwald et al. (1997);

A92:Acker et al. (1992); W83:Webster (1983); T92:Tylenda et al. (1992)

\begin{tabular}{ccc}
\hline \hline Distância da fonte central & Formato & Valores \\
\hline Função do ângulo polar $\theta$ & $R_{0} / \cos \theta$ & $R_{0}: 2 \mathrm{e} 16 \mathrm{~cm} ; \theta_{\text {max }}: 70^{\circ}$ \\
\hline & & \\
\hline Densidades & Formato & Valores \\
\hline $\begin{array}{c}\text { Distribuição angular } \\
\text { Variação radial }\end{array}$ & dfact $\times($ Dayal et al., 2000$)$ & dfact: 0,04 \\
\hline
\end{tabular}

Tabela 4.4 - Propriedades geométricas do modelo de IC4406. 


\subsubsection{NGC5844}

NGC5844 é uma extensa NP de simetria bipolar. Apesar de registrada no catálogo NGC, este objeto é comumente referido como Hen2-119. Um imageamento de média resolução encontra-se no quadro esquerdo da figura 4.4 (R. Corradi (Górny et al., 1999)).

Devido a sua grande extensão, três fendas foram posicionadas perpendicularmente ao eixo de simetria (quadro direito da figura 4.4). Tal posicionamento permite avaliar boa parte da região central brilhante. O avermelhamento registrado em cada região das fendas está na figura 4.5 .
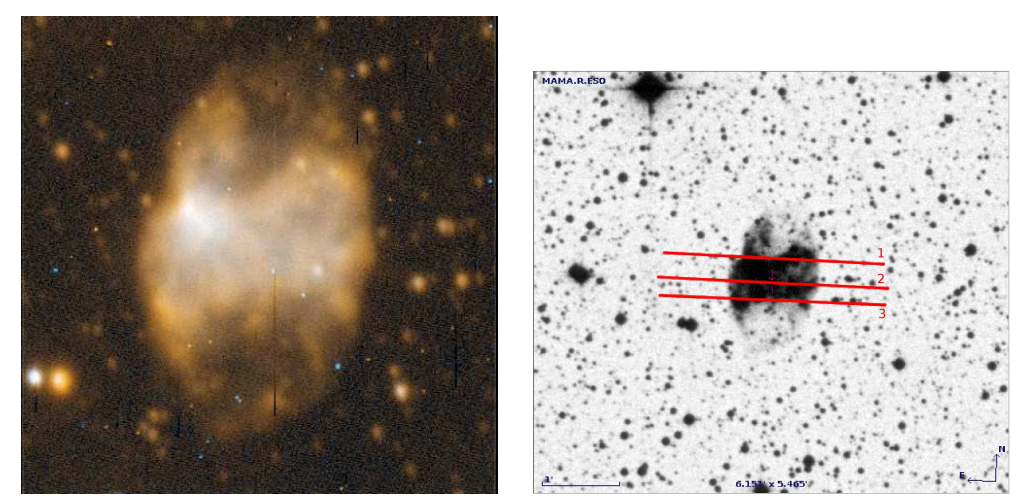

Figura 4.4: Imageamento de NGC5844 à esquerda; cores R:G:B = $\log [\mathrm{NII}]: \operatorname{ambos}: \log [\mathrm{OIII}]$. Indicação do posicionamento das fendas em imagem do cátalogo ESO.R-MAMA à direita.
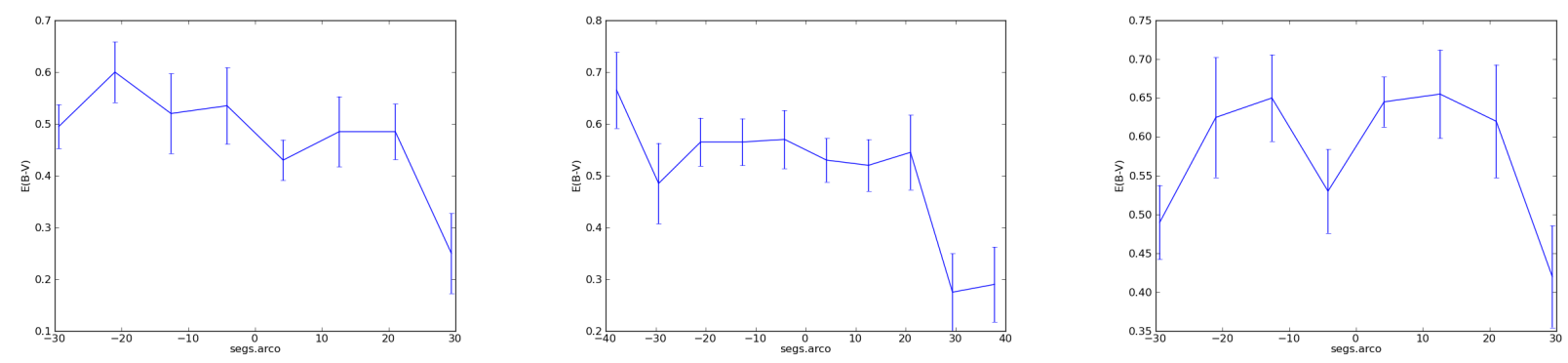

Figura 4.5: Variação do parâmetro de avermelhamento E(B-V) ao longo das fendas de NGC5844. Da esquerda para direita, fenda da posição superior, central e inferior, respectivamente.

Na tabela 4.5 estão os valores dos parâmetros físicos médios e da composição química, 
obtidos pela integração dos fluxos em toda a fenda colocada sobre a região central do objeto (de $\mathrm{n}^{\circ} .2$ ). Os valores da composição química obtidos foram os utilizados nas simulações. Os fluxos obtidos e as incertezas correspondentes estão no apêndice C.1.

Tabela 4.5 - Parâmetros físicos e composição química obtidos com integração por toda a fenda da região central de NGC5844.

\begin{tabular}{ccc|ccc}
\hline \hline Parâmetro & Valor & $\sigma$ & Parâmetro & Valor & $\sigma$ \\
\hline $\bar{n}_{e}$ & 260 & 209 & $\mathrm{He}^{+} / \mathrm{H}$ & 0,125 & 0,017 \\
$\bar{T}_{[N I I]}$ & 9351 & 1169 & $\mathrm{He}^{++} / \mathrm{H}$ & 0,018 & 0,002 \\
$\bar{T}_{[\text {OIII }]}$ & 8739 & 840 & $\mathrm{O}^{+} / \mathrm{H}$ & $1,4 \mathrm{e}-04$ & $9,8 \mathrm{e}-05$ \\
$\mathrm{He} / \mathrm{H}$ & 0,143 & 0,017 & $\mathrm{O}^{++} / \mathrm{H}$ & $5,1 \mathrm{e}-04$ & $2,0 \mathrm{e}-04$ \\
$\epsilon(\mathrm{O})$ & 8,87 & 0,15 & $\mathrm{~N}^{+} / \mathrm{H}$ & $8,9 \mathrm{e}-05$ & $3,2 \mathrm{e}-05$ \\
$\epsilon(\mathrm{N})$ & 8,69 & 0,16 & $\mathrm{~S}^{+} / \mathrm{H}$ & $2,4 \mathrm{e}-06$ & $8,5 \mathrm{e}-07$ \\
$\epsilon(\mathrm{S})$ & 7,19 & 0,29 & $\mathrm{~S}^{++} / \mathrm{H}$ & $9,3 \mathrm{e}-06$ & $6,9 \mathrm{e}-06$ \\
$\epsilon(\mathrm{Ne})$ & 8,71 & 0,18 & $\mathrm{Ne}^{3+} / \mathrm{H}$ & $3,5 \mathrm{e}-04$ & $1,6 \mathrm{e}-04$ \\
$\epsilon(\mathrm{Ar})$ & 6,89 & 0,11 & $\mathrm{Ar}^{3+} / \mathrm{H}$ & $3,9 \mathrm{e}-06$ & $1,2 \mathrm{e}-06$ \\
\hline
\end{tabular}

Este é um objeto para o qual apresentamos um modelo com limitação por ionização. Como a maioria dos modelos sob esta hipótese neste trabalho, o fluxo $\mathrm{H} \beta$ total é superestimado (neste caso, em mais de uma ordem de grandeza).

Simulações que possam reproduzir o fluxo total registrado por Acker et al. (1992) são desafiadores para NGC5844. Exploraram-se numerosos modelos de limitação por densidade, entretanto só foi possível reproduzir a emissão $H \beta$ a menos de um fator cinco.

$\mathrm{O}$ valor do fluxo $H \beta$ total do objeto parece ser único na literatura, e ainda com 0,3 dex de erro devido às grandes dimensões angulares. Seu valor (de ordem de $10^{-12} \mathrm{erg} / \mathrm{cm}^{2} \mathrm{~s}$ ) é surpreendentemente pequeno para um objeto destas dimensões. Uma outra medida deste fluxo seria de grande valor para avaliar os modelos.

Uma característica do modelo final é a baixa temperatura eletrônica observada, mesmo utilizando uma maior temperatura efetiva para a fonte central que o valor de literatura (tabela 4.8). Sistematicamente em modelos com limitação por densidade, as temperaturas eletrônicas registradas são maiores do que para aqueles limitados por ionização. Este é 
Tabela 4.6 - Parâmetros físicos e composição química obtidos com integração por toda a fenda da região superior de NGC5844. Valores compatíveis com região inferior.

\begin{tabular}{ccc|ccc}
\hline \hline Parâmetro & Valor & $\sigma$ & Parâmetro & Valor & $\sigma$ \\
\hline $\bar{n}_{e}$ & 247 & 74 & $\mathrm{He}^{+} / \mathrm{H}$ & 0,114 & 0,011 \\
$\bar{T}_{[N I I]}$ & 8335 & 1327 & $\mathrm{He}^{++} / \mathrm{H}$ & 0,012 & 0,001 \\
$\bar{T}_{[O I I I]}$ & 8530 & 1084 & $\mathrm{O}^{+} / \mathrm{H}$ & $4,4 \mathrm{e}-04$ & $4,4 \mathrm{e}-04$ \\
$\mathrm{He} / \mathrm{H}$ & 0,126 & 0,011 & $\mathrm{O}^{++} / \mathrm{H}$ & $5,1 \mathrm{e}-04$ & $2,6 \mathrm{e}-04$ \\
$\epsilon(\mathrm{O})$ & 9,02 & 0,26 & $\mathrm{~N}^{+} / \mathrm{H}$ & $1,5 \mathrm{e}-04$ & $8,3 \mathrm{e}-05$ \\
$\epsilon(\mathrm{N})$ & 8,57 & 0,13 & $\mathrm{~S}^{+} / \mathrm{H}$ & $3,9 \mathrm{e}-06$ & $2,0 \mathrm{e}-06$ \\
$\epsilon(\mathrm{S})$ & 7,05 & 0,4 & $\mathrm{~S}^{++} / \mathrm{H}$ & $6,6 \mathrm{e}-06$ & $6,6 \mathrm{e}-06$ \\
$\epsilon(\mathrm{Ne})$ & 8,96 & 0,28 & $\mathrm{Ne}^{3+} / \mathrm{H}$ & $4,5 \mathrm{e}-04$ & $2,8 \mathrm{e}-04$ \\
$\epsilon(\mathrm{Ar})$ & 6,96 & 0,24 & $\mathrm{Ar}^{3+} / \mathrm{H}$ & $3,3 \mathrm{e}-06$ & $1,1 \mathrm{e}-06$ \\
\hline
\end{tabular}

também um fator a favor de que a matéria tenha uma distribuição mais complexa na nuvem, que pode ou não estar associada com a limitação por densidade (ao menos em regiões do objeto).

Das três fendas observadas (figuras 4.6 à 4.8), a fenda da região inferior (de $\mathrm{n}^{\mathrm{o}}$. 3) foi a que apresentou maior dispersão e desvio dos dados observacionais, em boa parte devido ao menor fluxo proveniente da região observada em relação às outras fendas. Outra possibilidade é a presença de não-homogeneidades, que não podem ser determinadas em virtude dos erros associados. 
Tabela 4.7 - Parâmetros físicos e composição química previstos pelo modelo de NGC5844, e respectivo valor de $\chi^{2}$ em relação às observações. Dada a simetria do modelo, são exibidos somente os valores até a janela central.

\begin{tabular}{ccccccc}
\hline \hline Parâmetro & $\chi^{2}$ & Janela 1 & Janela 2 & Janela 3 & Janela 4 & Janela 5 \\
\hline $\bar{n}_{e}$ & 1,71 & 216,86 & 283,43 & 284,86 & 287,67 & 276,23 \\
$\bar{T}_{[N I I]}$ & 7,27 & 8920,12 & 8764,17 & 8725,46 & 8781,74 & 8911,04 \\
$\bar{T}_{[O I I I]}$ & 16,81 & 9179,24 & 8394,97 & 7810,47 & 7831,08 & 8601,57 \\
$\mathrm{He}^{+} / \mathrm{H}$ & 21,7 & 0,208 & 0,157 & 0,153 & 0,148 & 0,124 \\
$\mathrm{He}^{++} / \mathrm{H}$ & 31,31 & 0,0 & 0,0 & 0,0 & 0,0021 & 0,029 \\
$\mathrm{He} / \mathrm{H}$ & 16,23 & 0,208 & 0,157 & 0,153 & 0,151 & 0,152 \\
$\mathrm{O}^{+} / \mathrm{H}$ & 16,04 & $7,50 \mathrm{E}-4$ & $2,80 \mathrm{E}-4$ & $1,10 \mathrm{E}-4$ & $7,60 \mathrm{E}-5$ & $6,30 \mathrm{E}-5$ \\
$\mathrm{O}^{++} / \mathrm{H}$ & 12,34 & $7,20 \mathrm{E}-5$ & $4,60 \mathrm{E}-4$ & $5,80 \mathrm{E}-4$ & $6,00 \mathrm{E}-4$ & $4,00 \mathrm{E}-4$ \\
$\mathrm{fci} O$ & - & 1 & 1 & 1 & 1,01 & 1,23 \\
$\epsilon(\mathrm{O})$ & 7,8 & 8,92 & 8,87 & 8,84 & 8,84 & 8,76 \\
$\mathrm{~N}^{+} / \mathrm{H}$ & 19,36 & $5,80 \mathrm{E}-4$ & $1,90 \mathrm{E}-4$ & $7,30 \mathrm{E}-5$ & $5,00 \mathrm{E}-5$ & $4,30 \mathrm{E}-5$ \\
$\mathrm{fci}$ & - & 1,1 & 2,64 & 6,3 & 9,01 & 8,99 \\
$\epsilon(\mathrm{N})$ & 15,18 & 8,8 & 8,7 & 8,66 & 8,65 & 8,59 \\
$\mathrm{~S}^{+} / \mathrm{H}$ & 24,58 & $1,70 \mathrm{E}-5$ & $4,00 \mathrm{E}-6$ & $1,60 \mathrm{E}-6$ & $1,10 \mathrm{E}-6$ & $9,60 \mathrm{E}-7$ \\
$\mathrm{~S}^{++} / \mathrm{H}$ & 7,14 & $6,80 \mathrm{E}-6$ & $1,20 \mathrm{E}-5$ & $1,10 \mathrm{E}-5$ & $7,90 \mathrm{E}-6$ & $4,40 \mathrm{E}-6$ \\
$\mathrm{fci}{ }_{S}$ & - & 1 & 1,31 & 2,47 & 3,36 & 3,36 \\
$\epsilon(\mathrm{S})$ & 15,09 & 7,37 & 7,33 & 7,5 & 7,48 & 7,25 \\
$\mathrm{Ne}^{3+} / \mathrm{H}$ & 26,03 & $5,60 \mathrm{E}-4$ & $5,40 \mathrm{E}-4$ & $5,30 \mathrm{E}-4$ & $5,10 \mathrm{E}-4$ & $3,50 \mathrm{E}-4$ \\
$\mathrm{fci}_{N e}$ & - & 11,53 & 1,61 & 1,19 & 1,14 & 1,43 \\
$\epsilon(\mathrm{Ne})$ & 20,07 & 9,81 & 8,94 & 8,8 & 8,77 & 8,7 \\
$\mathrm{Ar}^{3+} / \mathrm{H}$ & 36,4 & $8,70 \mathrm{E}-6$ & $7,80 \mathrm{E}-6$ & $6,60 \mathrm{E}-6$ & $5,00 \mathrm{E}-6$ & $3,30 \mathrm{E}-6$ \\
$\mathrm{fci}_{A r}$ & - & 15,46 & 2,16 & 1,59 & 1,53 & 1,91 \\
$\epsilon(\mathrm{Ar})$ & 25,55 & 8,13 & 7,23 & 7,02 & 6,89 & 6,8 \\
$\mathrm{E}(\mathrm{B}-\mathrm{V})$ & - & & & & & \\
\hline & & & & & & \\
\hline
\end{tabular}


Tabela 4.8 - Parâmetros do modelo final de NGC5844 comparados com referências.

\begin{tabular}{cccc}
\hline \hline Parâmetro & Modelo & Valores de Ref. & Referências \\
\hline $10^{3} \cdot T_{\text {eff }}(\mathrm{K})$ & 90 & 78,8 & $\mathrm{Z} 93$ \\
$\mathrm{~L} / \mathrm{L}_{\odot}$ & 3630 & 3600 & $\mathrm{Z} 93$ \\
Espectro & $\mathrm{H}-\mathrm{Ni}$ & - & - \\
$\log \mathrm{g}\left(\mathrm{cm} / \mathrm{s}^{2}\right)$ & 5,2 & 5,2 & $\mathrm{Z} 93$ \\
$\bar{E}(B-V)$ & 0,41 & 0,41 & $\mathrm{~T} 92$ \\
$\mathrm{~F}_{H \beta}^{\text {total }}\left(\mathrm{erg} / \mathrm{cm}^{2} \mathrm{~s}\right)$ & $1,31 \mathrm{E}-10$ & $6,31 \mathrm{E}-12$ & $\mathrm{~A} 92$ \\
$\mathrm{~d}(\mathrm{kpc})$ & 2,44 & $0,97 \leq 1,7$ & $\mathrm{~A} 92$ \\
$\mathrm{M}_{\text {ion }} / \mathrm{M}_{\odot}$ & 2,5 & - & - \\
$i(\mathrm{grau})$ & $90^{\circ}$ & - & - \\
\hline Z93:Zhang e Kwok (1993); T92:Tylenda et al. (1992); A92:Acker et al. (1992)
\end{tabular}

Tabela 4.9 - Propriedades geométricas do modelo de NGC5844.

\begin{tabular}{ccc}
\hline \hline Distância da fonte central & Formato & Valores \\
\hline Função do ângulo polar $\theta$ & $R_{0} / \cos \theta$ & $R_{0}: 1 \mathrm{e} 16 \mathrm{~cm} ; \theta_{\text {max }}: 50^{\circ}$ \\
\hline & & \\
\hline Densidades & Formato & Valores \\
\hline Distribuição angular & dfact $\times($ Dayal et al., 2000) & dfact: 0,01 \\
Variação radial & nula & - \\
\hline
\end{tabular}




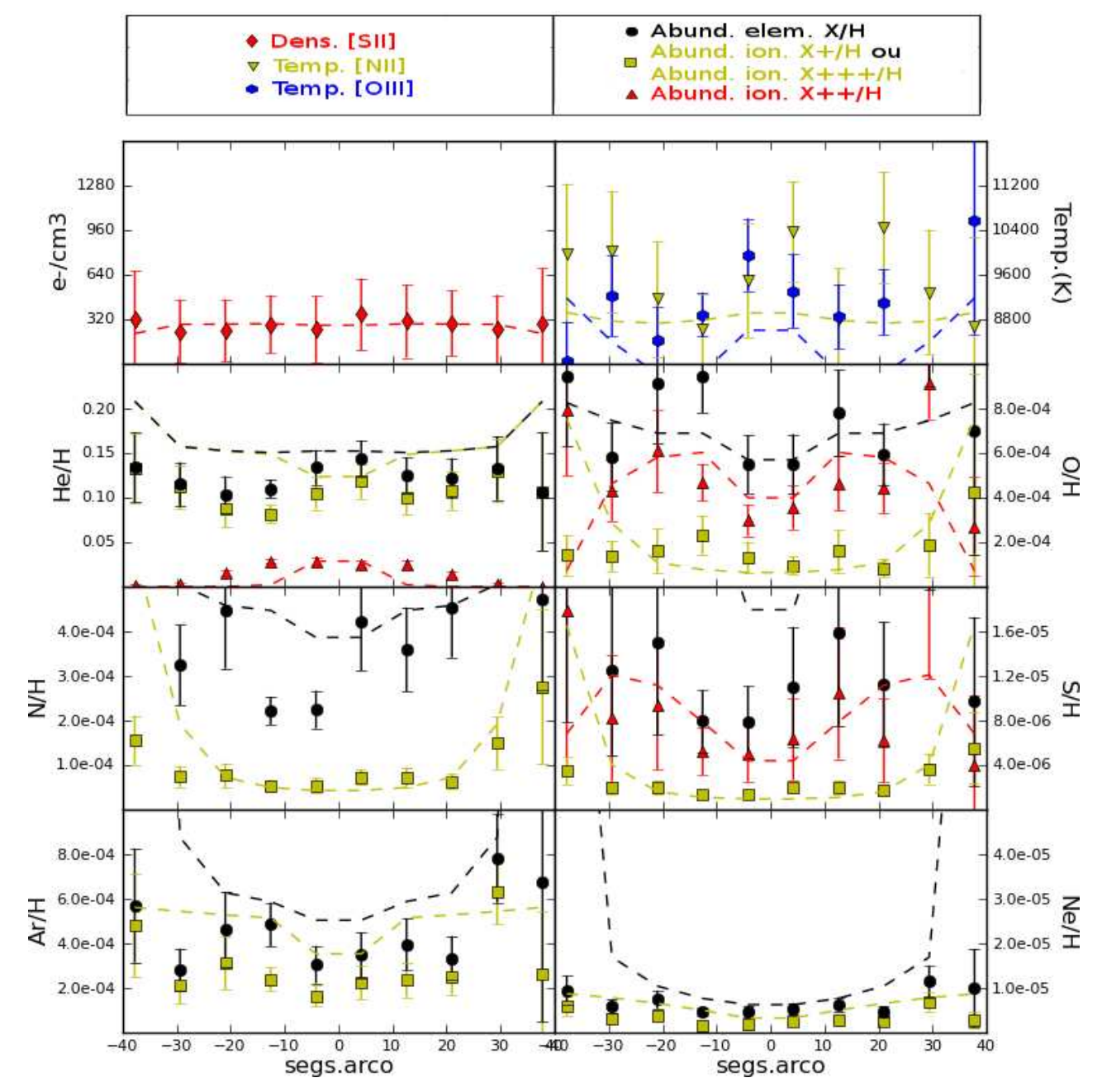

Figura 4.6: Parâmetros físicos e composição química obtidos para NGC5844 (pontos) e respectiva previsão do modelo (linha tracejada). Fenda central $\left(n^{\circ} 2\right)$. 


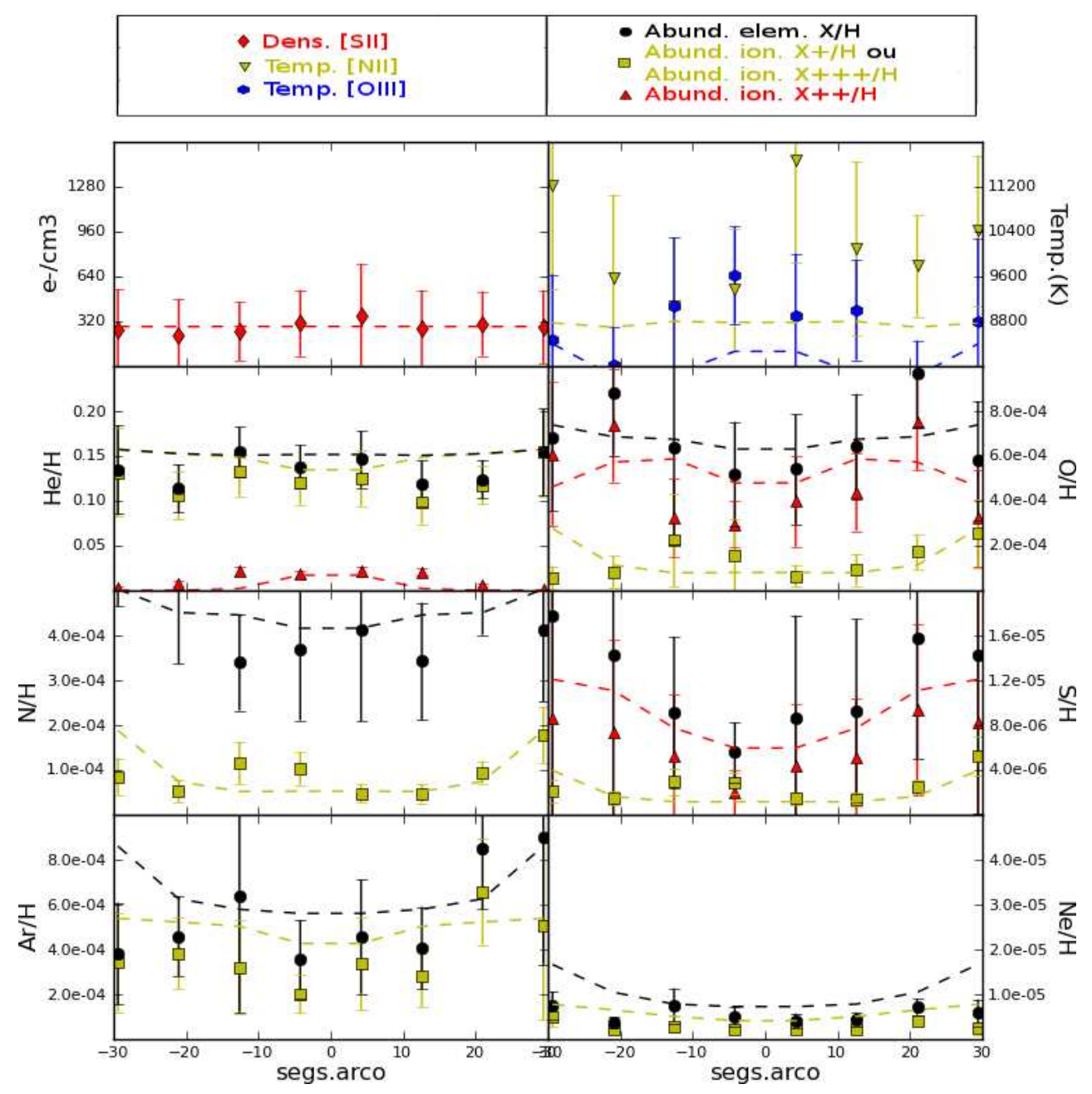

Figura 4.7: Parâmetros físicos e composição química obtidos para NGC5844 (pontos) e respectiva previsão do modelo (linha tracejada). Fenda superior $\left(n^{\circ} 1\right)$. 


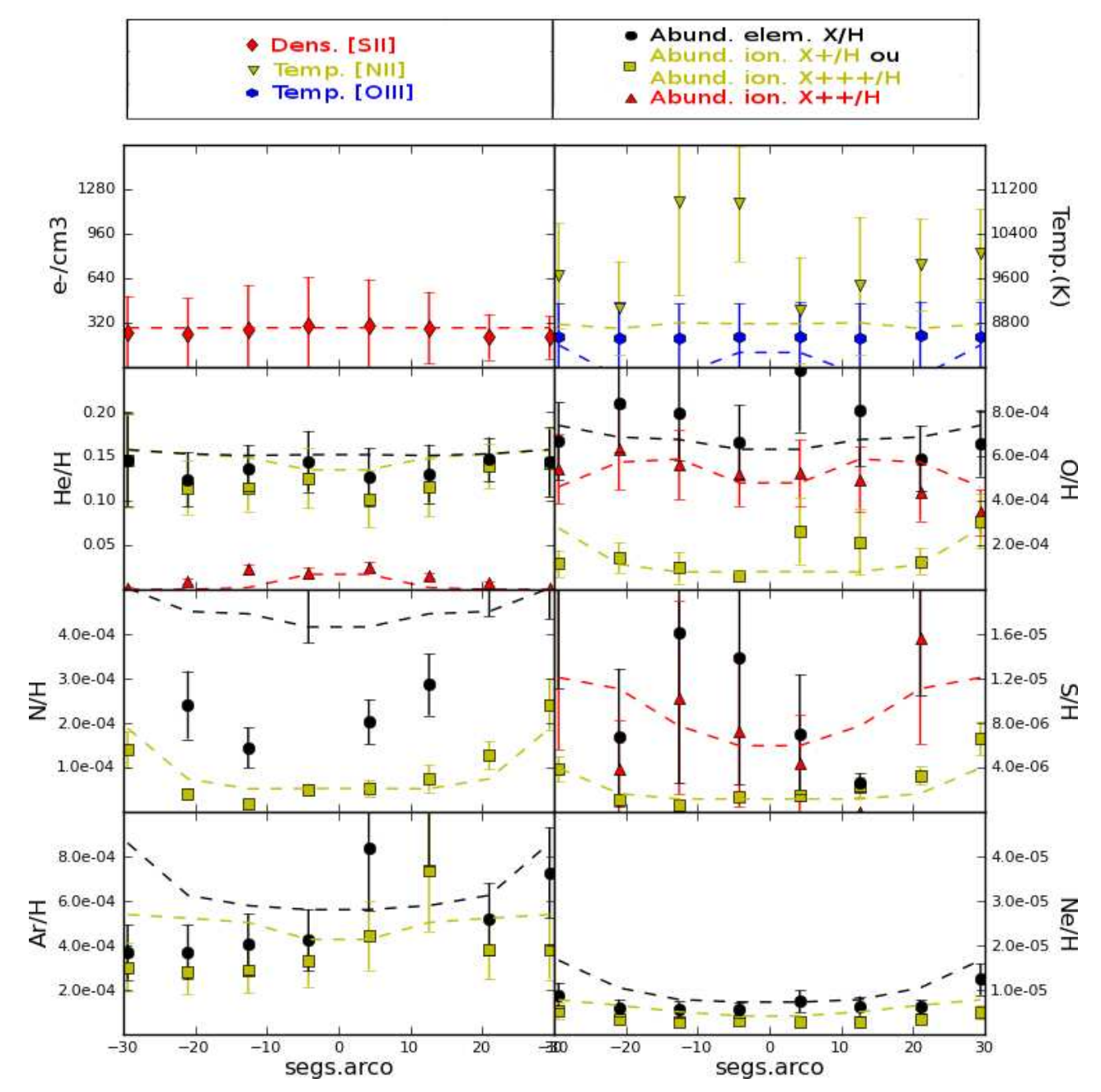

Figura 4.8: Parâmetros físicos e composição química obtidos para NGC5844 (pontos) e respectiva previsão do modelo (linha tracejada). Fenda inferior $\left(n^{\circ} 3\right)$. 


\subsection{Espectroscopia de campo integral}

\subsubsection{NGC6572}

NGC6572 é uma NP de alto brilho superficial e amplamente estudada (figura 4.9, de B. Bodner ${ }^{1}$ ). No trabalho de Hyung et al. (1994) é proposto um modelo bipolar bicônico para este objeto, com $i=45^{\circ}$.

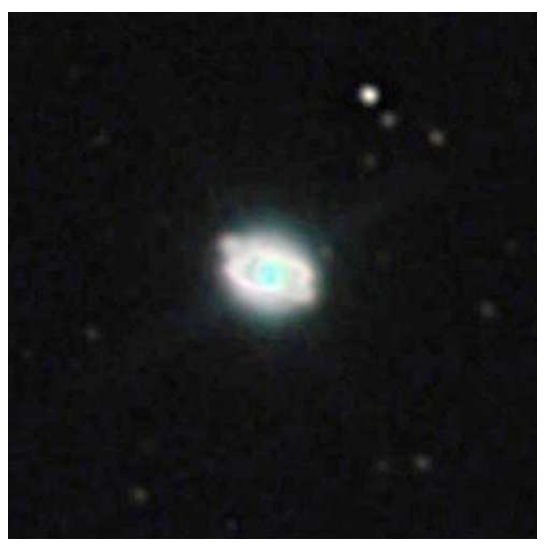

Figura 4.9: Imageamento de NGC6572. Coloração e orientação desconhecidos.

\begin{tabular}{lr}
\hline \hline Parâmetro & Valor adotado \\
\hline $\mathrm{He} / \mathrm{H}$ & 0,110 \\
$\epsilon(\mathrm{O})$ & 8,60 \\
$\epsilon(\mathrm{N})$ & 8,31 \\
$\epsilon(\mathrm{S})$ & 6,52 \\
$\epsilon(\mathrm{Ne})$ & 7,93 \\
$\epsilon(\mathrm{Ar})$ & 6,33 \\
\hline
\end{tabular}

Tabela 4.10 - Valores de composição química de NGC6572 adotados para as simulações.

Este modelo, apesar de reproduzir alguns dos fluxos esperados para o objeto, não é consistente com os dados observacionais obtidos com a técnica de espectroscopia de campo integral. Em especial, numa geometria como a proposta espera-se um núcleo bem compacto e de mais alta densidade em virtude da proximidade com a fonte central, como observado no objeto MyCn18 (Zepeda, 2006).

\footnotetext{
${ }^{1}$ Disponível no endereço eletrônico http://www.noao.edu/outreach/aop/observers/observer123.html .
} 
Um modelo com geometria elíptica é aqui proposto e pode reproduzir adequadamente as características das variações nas razões de fluxos. Os altos valores de [NII], principalmente na região equatorial, sugerem a presença de baixa intensidade de radiação, enquanto que os altos valores de densidade eletrônica indicam o cenário oposto.

Tais regiões distintas podem entretanto ser vistas numa mesma linha de visada, sob a hipótese de variação na densidade eletrônica em função da distância à fonte central: após transpassar uma camada de alta densidade, a radiação emergente interage com material em baixa densidade ou em menor intensidade, gerando maior emissão de [NII] excitado colisionalmente em oposição à emissão $\mathrm{H} \alpha$, emitido em recombinação eletrônica e sua cascata após ionização.

Um modelo elíptico para este objeto, limitado por ionização, é proposto por Sabbadin et al. (2006). O ângulo com a linha de visada é determinado neste modelo em $i \sim 80^{\circ}$ (comunicação particular). A composição química utilizada está indicada na tabela 4.10, obtida de Girard et al. (2007).

Tabela 4.11 - Parâmetros do modelo final de NGC6572 comparados com referências.

\begin{tabular}{cccc}
\hline \hline Parâmetro & Modelo & Valores de Ref. & Referências \\
\hline $\bar{n}_{e}\left(e^{-} / \mathrm{cm}^{3}\right)$ & 13500 & 17410 & $\mathrm{G} 07$ \\
$\bar{T}_{[N I I]}(\mathrm{K})$ & 10188 & 10200 & $\mathrm{G} 07$ \\
$\bar{T}_{[O I I I]}(\mathrm{K})$ & - & 11700 & $\mathrm{G} 07$ \\
$10^{3} \cdot T_{e f f}(\mathrm{~K})$ & 65 & 64,$6 ; 56,4 ; 63,3$ & $\mathrm{GP} 89 ; \mathrm{Z} 93 ; \mathrm{M} 97$ \\
$\mathrm{~L} / \mathrm{L} \odot$ & 3981 & $245 ; 8300 ; 7100$ & $\mathrm{GP} 89 ; \mathrm{Z} 93 ; \mathrm{M} 97$ \\
$\mathrm{Espectro}$ & $\mathrm{PG} 1159$ & Of/WR; wels & $\mathrm{A} 92 ; \mathrm{G} 07$ \\
$\log \mathrm{g}\left(\mathrm{cm} / \mathrm{s}^{2}\right)$ & 4,5 & 4,$3 ; 4,55$ & $\mathrm{Z} 93 ; \mathrm{M} 97$ \\
$\bar{E}(B-V)$ & 0,4 & 0,$3 ; 0,4$ & $\mathrm{G} 07 ; \mathrm{H} 94$ \\
$\mathrm{~F}_{H \beta}^{\text {total }}\left(\mathrm{erg} / \mathrm{cm}^{2} \mathrm{~s}\right)$ & $3,87 \mathrm{E}-010$ & $3,80 \mathrm{E}-010$ & $\mathrm{~A} 92$ \\
$\mathrm{~d}(\mathrm{kpc})$ & 1,87 & 2,4 & $\mathrm{M} 97$ \\
$\mathrm{M}_{\text {ion }} / \mathrm{M}_{\odot}$ & 0,1 & - & - \\
$i(\mathrm{grau})$ & $80^{\circ}$ & $80^{\circ}$ & (ver texto) \\
\hline
\end{tabular}

G07:Girard et al. (2007); GP89:Gathier e Pottasch (1989); Z93:Zhang e Kwok (1993); M97:Mal'Kov (1997); A92:Acker et al. (1992); H94:Hyung et al. (1994). 
Basicamente, a distribuição espacial dos fluxos (e consequentemente dos fluxos globais) dependerá da distribuição de matéria em torno da fonte de radiação (estrela central). Sob as hipóteses discutidas neste trabalho, duas características tornam-se importantes: a distância da material à fonte central e sua dependência radial. Tais características não podem ser diretamente observadas, e portanto as variações destas configurações são limitadas a fim de se garantir um padrão nas simulações.

Um modelo simples, e que produziu excelentes resultados, foi gerado a partir das seguintes hipóteses: a densidade de Hidrogênio é constante numa dada direção, e a distância do material nas diferentes direções é fixa. Os parâmetros do modelo final estão na tabela 4.11, e os mapas de razões de fluxos na figura 4.11. A distribuição de matéria é dada pela equação 3.2. As propriedades geométricas da nuvem estão sintetizadas na tabela 4.12.

Tabela 4.12 - Propriedades geométricas do modelo de NGC6572.

\begin{tabular}{ccc}
\hline \hline Distância da fonte central & Formato & Valores \\
\hline Função do ângulo polar $\theta$ & constante & $r_{0}: 5 \mathrm{e} 16 \mathrm{~cm}$ \\
\hline Densidades & Formato & Valores \\
\hline & & \\
\hline Distribuição angular & equação 3.2 & $m: 3 ; n_{0}: 4000 \mathrm{H} / \mathrm{cm}^{3} ; \epsilon: 2.7$ \\
Variação radial & nula & - \\
\hline
\end{tabular}

Tabela 4.13 - Valores de $\chi^{2}$ entre o modelo final e as observações de NGC6572.

\begin{tabular}{llll}
\hline \hline Mapas & $\chi_{\text {perp }}^{2}$ & $\chi_{\text {para }}^{2}$ & $\sigma$ típico \\
\hline $\mathrm{n}_{e}[\mathrm{SII}]$ & 102,0 & 82,8 & 1500 \\
$(\lambda 6548+6584) / \mathrm{H} \alpha$ & 162,6 & 98,9 & 0,04 \\
$\log [\lambda 6312 /(\lambda 6716+6731)]$ & 74,5 & 33,0 & $-0,1$ \\
$\log [(\lambda 6716+6731) / \lambda 6312]$ & 20,9 & 12,9 & 0,25 \\
\hline
\end{tabular}

Na tabela 4.13 temos os valores de $\chi^{2}$ entre os resultados do melhor modelo para NGC6572 e seus dados observacionais, com respectiva incerteza na variação dos fluxos 
observados. O pequeno erro associado aos fluxos é reflexo do alto brilho superficial do objeto. Como o objeto possui dimensões de $\sim 12 \mathrm{x} 17 "$, temos que $\chi^{2} / N \sim 8$, onde $N$ é a quantidade de pontos observacionais modelados. Este valor é razoável, uma vez que as observações possuem pequeno erro associado, com um modelo que não prevê inomogeneidades esperadas num meio turbulento como o esperado pelo modelo de ventos interagentes.
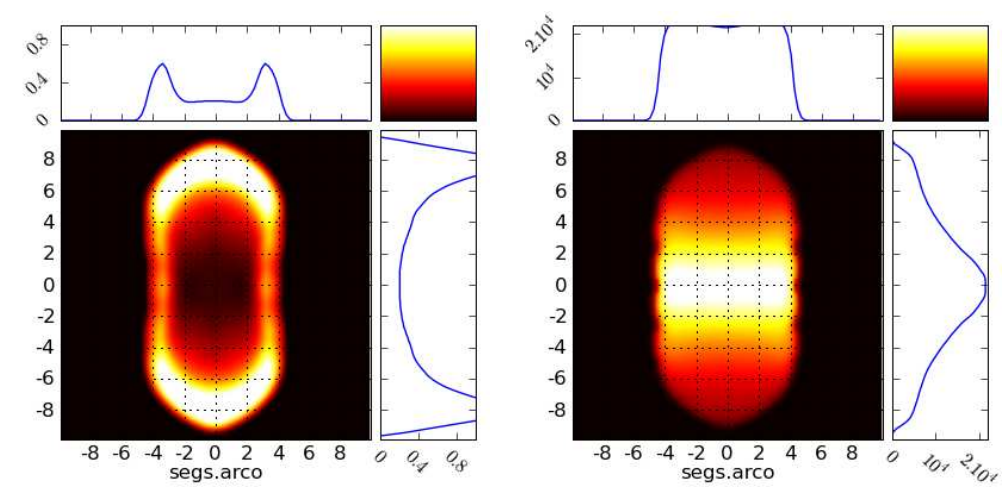

Figura 4.10: Mapas de melhor ajuste em outros modelos considerados de NGC6572. Apesar da maior similaridade, os modelos de tais mapas falham na reprodução global dos vínculos considerados.

Para o objeto NGC6572, esta simples representação produziu bons resultados, mesmo considerando-se fixa a distância do material em relação à fonte central. É realmente esperado que esta hipótese gere grandes diferenças se $\Delta R \gg R_{0}$, como no caso deste objeto.

Para comparação, colocamos na figura 4.10 o resultado de outros dois modelos com mapas de menor $\chi^{2}$ para NGC6572 em densidade S[II] (à esquerda) e de razão [NII]/H $\alpha$ (à direita), do conjunto de modelos desenvolvidos. Os valores de $\chi_{\text {perp }}^{2}$ e $\chi_{\text {para }}^{2}$ são 46,2, 35,4 e 36,0, 63,5, respectivamente (aproximadamente fatores 0,3 e 0,5 em relação ao melhor modelo global).

Apesar do melhor ajuste individualmente nestes mapas, outros vínculos são quebrados nestes modelos. Por exemplo, no mapa $[\mathrm{NII}] / \mathrm{H} \alpha$, o alto contraste entre a região central e as regiões periféricas pode ser reproduzido se aumentada a luminosidade $\left(\mathrm{L}_{\mathrm{L}} \mathrm{L}_{\odot}=7940\right)$ e diminuída a temperatura efetiva $\left(\mathrm{T}_{e f f}=61.500 \mathrm{~K}\right)$ da estrela central. Entretanto, tal mudança tem por efeito um acréscimo no fluxo $\mathrm{H} \beta$ observado em $34 \%$ nas mesmas considerações geométricas.

O mapa de densidade exibido na figura 4.10 pode ser obtido se diminuida a luminosidade 
$\left(\mathrm{L} / \mathrm{L}_{\odot}=1000\right)$ e a temperatura efetiva $\left(\mathrm{T}_{e f f}=55.000 \mathrm{~K}\right)$ da fonte central. Tais diferenças em relação ao melhor modelo devem refletir de uma distribuição não constante de matéria sob a casca. O caso da densidade [SII] ser melhor reproduzida por uma fonte central de menos fótons ionizantes pode se associado com a localização da região emissora das linhas $\mathrm{S}[\mathrm{II}]$ em regiões de menor fluxo radiativo que o modelo final, por exemplo, com uma maior densidade interna de material do que na região externa. 

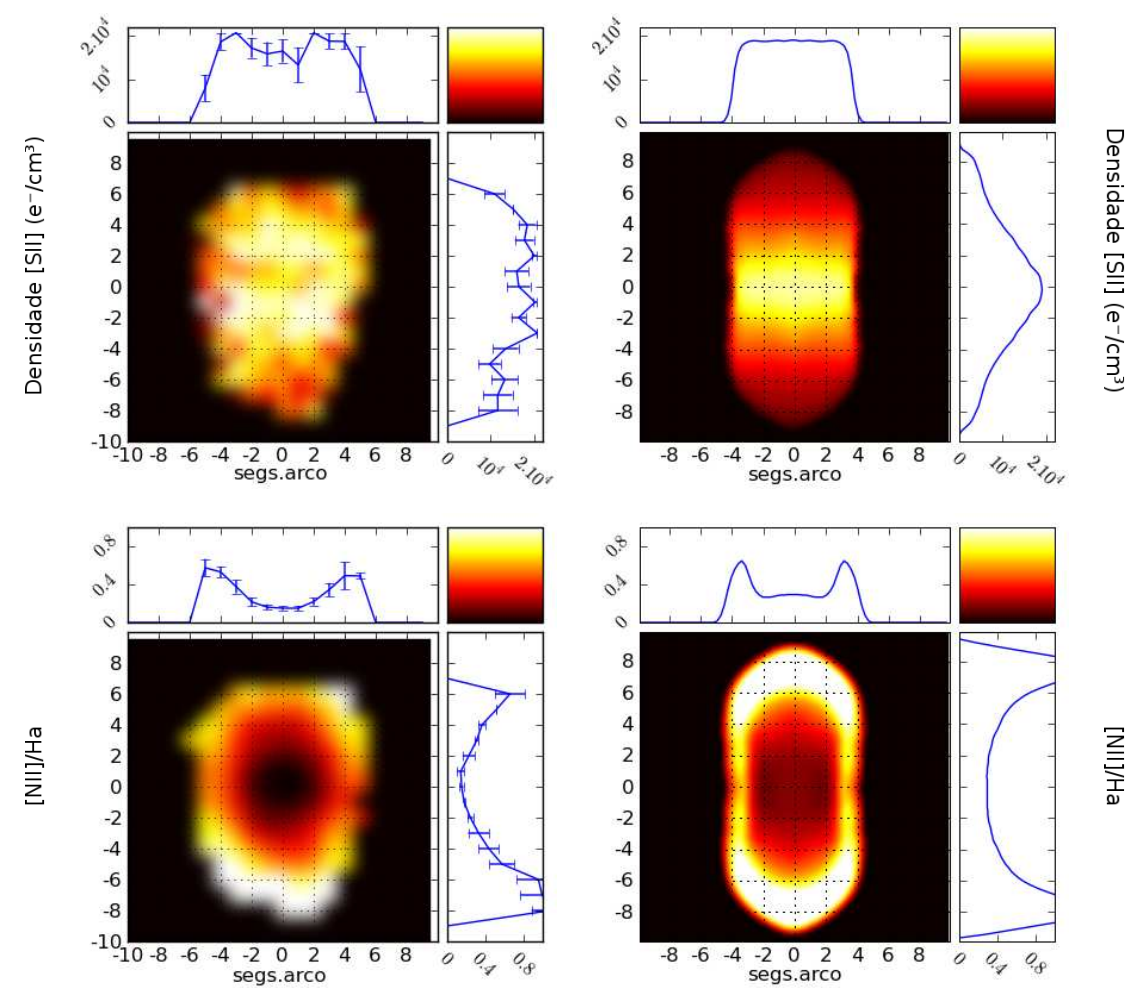

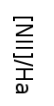
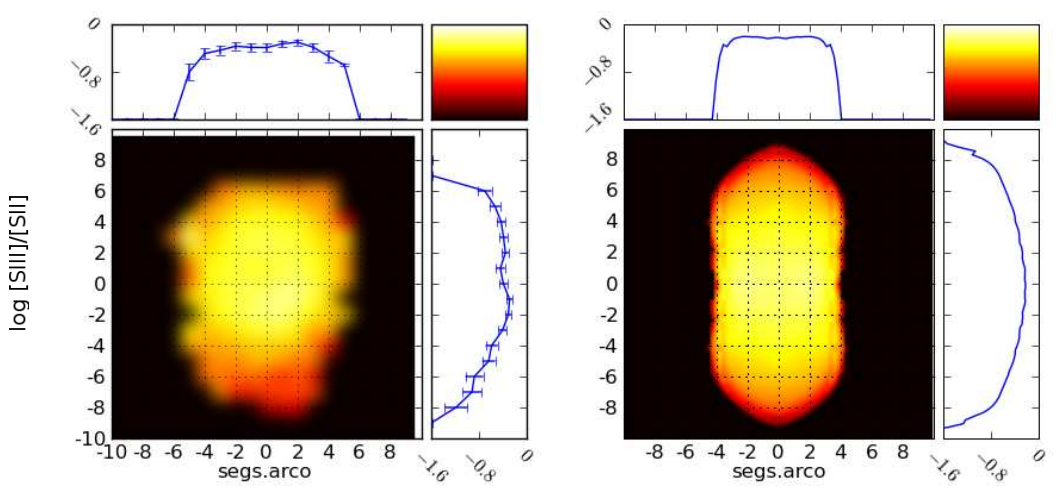

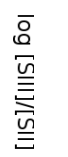
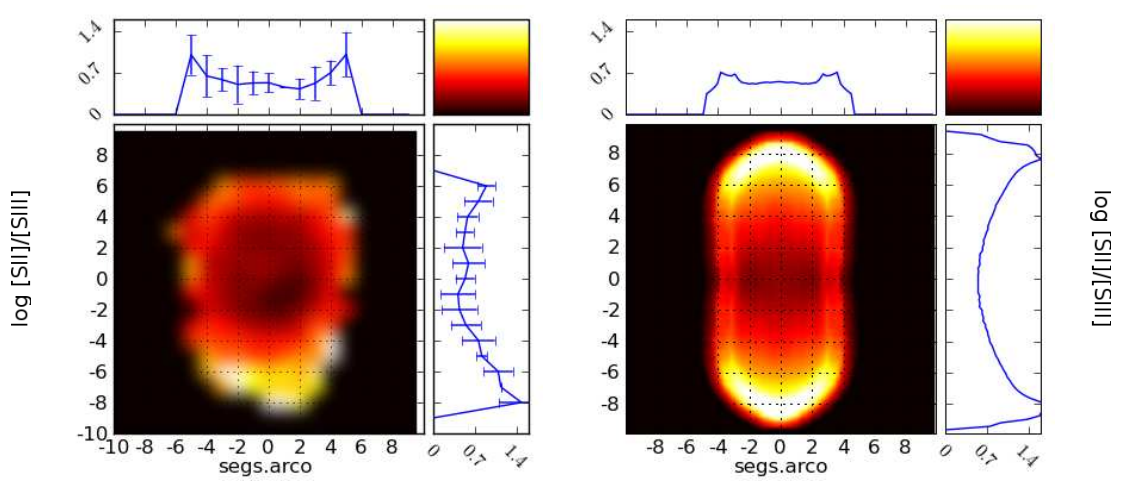

Figura 4.11: Comparação entre os dados observacionais de NGC6572 (à esquerda) e as respectivas previsões do modelo (à direita). 


\subsubsection{IC2553}

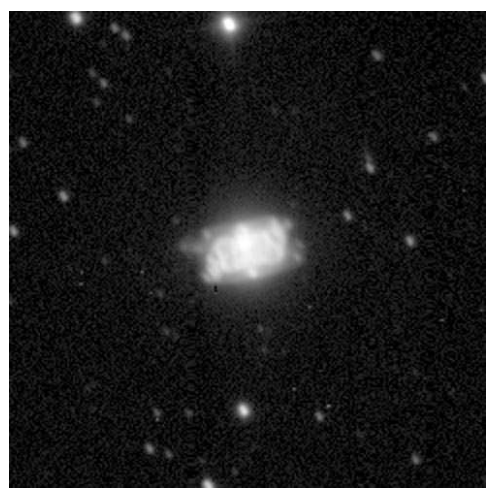

Figura 4.12: Imageamento de IC2553. Intensidade $\log ([\mathrm{NII}]+\mathrm{H} \alpha)$. Orientação arbitrária.

O formato aproximadamente retangular deste objeto (figura 4.12, R. Corradi (Schwarz et al., 1992)), similar a IC4406, e o contraste de densidades entre equador e pólos são fatores indicativos para a geometria em forma de tubo. O ângulo com a linha de visada foi determinado por Corradi et al. (2000) como $i=78^{\circ}$ a partir de considerações cinemáticas do gás obtidas através de echelogramas. Nestas observações é também evidente a presença de uma sub-estrutura brilhante na região equatorial. Um dos diagramas posição-velocidade que evidencia as propriedades desta sub-estrutura é exibida na figura 4.13, adaptada de Corradi et al. (2000).

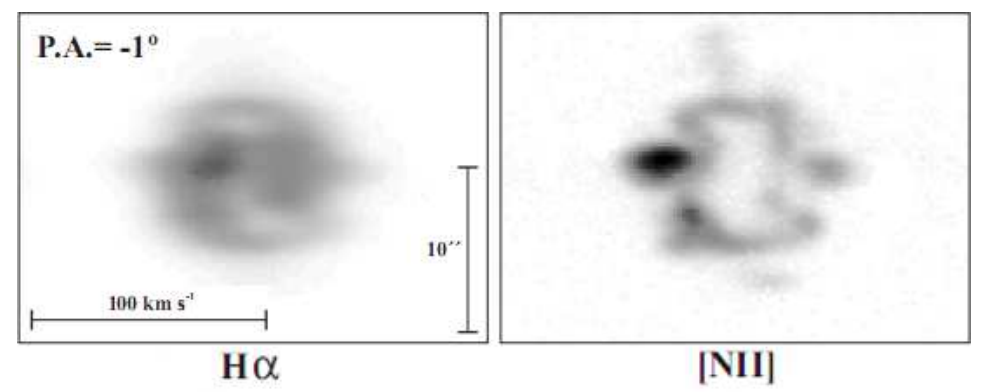

Figura 4.13: Diagrama posição-velocidade em escala logarítmica para IC2553. P.A. refere-se ao ângulo entre o posicionamento da instrumentação e eixo maior projetado do objeto.

Uma vez feita esta consideração sobre a geometria, a fraca emissão de [NII] no equador sugere que este objeto seja limitado por densidade e não por radiação nesta região (a radiação emergente seria capaz de excitar material com presença de íons $\mathrm{N}^{+}$, o que não ocorre em virtude da ausência de material). 
Tabela 4.14 - Valores de composição química de IC2553 adotados para as simulações.

\begin{tabular}{lr}
\hline \hline Parâmetro & Valor adotado \\
\hline $\mathrm{He} / \mathrm{H}$ & 0,105 \\
$\epsilon(\mathrm{O})$ & 8,80 \\
$\epsilon(\mathrm{N})$ & 8,28 \\
$\epsilon(\mathrm{Ne})$ & 8,14 \\
\hline
\end{tabular}

Distintos envoltórios de densidade constante foram modelados como frações da distribuições de Dayal et al. (2000), além de alguns modelos de densidade constante em cada direção, limitados por densidade. A composição química utilizada está descrita na tabela 4.14, obtida de Perinotto (1991). E nenhuma informação sobre a fonte central deste objeto foi encontrada na literatura.

O resultado destas simulações iniciais exibiram duas marcantes características: i) densidades constantes não reproduziram adequadamente a distribuição de densidades observada; ii) os fluxos $\mathrm{H} \beta$ totais, sob a hipótese de NP limitada por ionização ( "ionization bounded" é o termo empregado em inglês) apresentam pelo menos um fator 3 em relação ao fluxo observado.

Desta forma, o contraste na razão de fluxos de $[\mathrm{NII}] / \mathrm{H} \alpha$ entre o equador e os pólos, muito marcante neste objeto, é um indicativo não só da distribuição não homogênea de densidade, mas também da limitação por densidade em pelo menos parte destas regiões, justificando a ausência de forte emissão na razão $[\mathrm{NII}] / \mathrm{H} \alpha$ sob esta hipótese. Outro fator correlacionado com a limitação por ionização é a deteç̧ão de um halo em emissão para este objeto (Corradi et al., 2003).

Sob as considerações geométricas para este objeto, modelos limitados por densidade partiram da hipótese de única camada de densidade constante, mas de espessura fixa a cada direção. Tais modelos foram capazes de reproduzir o fluxo $\mathrm{H} \beta$ observado, porém levando a dois tipos de mapas $[\mathrm{NII}] / \mathrm{H} \alpha$ : de razão maior a 0,9 ou menor a 0,05 em toda a extensão do objeto. De uma forma geral, estes resultados surgem em função de um único parâmetro, o raio interno $R_{0}$; caso seu valor seja pequeno, temos alta intensidade de radiação incidente no material, e a emissão [NII] não é considerável. Para $R_{0}$ suficientemente grande, a 
radiação incidente é de menor intensidade com significativa emissão [NII].

Dentre os modelos limitados por densidade, foi escolhido o que melhor reproduzisse os outros mapas observados. Seus resultados são exibidos na figura 4.14, coluna central. Com as hipóteses consideradas para este cenário, o fluxo $\mathrm{H} \beta$ é mais facilmente reproduzido considerando o espectro da estrela central como uma estrela pobre em H (PG1159 - o que não necessariamente é sempre esperado).

Tabela 4.15 - Parâmetros dos modelos finais de IC2553 comparados com referências.

\begin{tabular}{lllll}
\hline \hline Parâmetro & Modelo LI & Modelo LD & Valores de Ref. & Referências \\
\hline $\bar{n}_{e}\left(e^{-} / \mathrm{cm}^{3}\right)$ & 10207 & 11191 & 8700 & P98 \\
$\bar{T}_{[N I I]}(\mathrm{K})$ & 9761 & 11048 & $10100 ; 10720$ & P98; MK96 \\
$\bar{T}_{[O I I I]}(\mathrm{K})$ & - & - & 9400 & MK96 \\
$10^{3} \cdot T_{\text {eff }}(\mathrm{K})$ & 98 & 115 & 99,$3 ; 150 ; 96,8$ & PM91; Z93; M97 \\
$\mathrm{L} / \mathrm{L} \odot$ & 400 & 3500 & $400 ; 6600$ & Z93; M97 \\
Espectro & $\mathrm{PG} 1159$ & $\mathrm{H}-\mathrm{Ni}$ & - & - \\
$\log \mathrm{g}\left(\mathrm{cm} / \mathrm{s}^{2}\right)$ & 7,4 & 7,4 & 7,4 & $\mathrm{M} 97$ \\
$\bar{E}(B-V)$ & 0,49 & 0,49 & 0,49 & $\mathrm{~T} 92$ \\
$\mathrm{~F}_{H \beta}^{t o t a l}(\mathrm{erg} / \mathrm{cm} . \mathrm{s})$ & $4,39 \mathrm{E}-011$ & $2,32 \mathrm{E}-010$ & $4,68 \mathrm{E}-011$ & A92 \\
$\mathrm{d}(\mathrm{kpc})$ & 1,69 & 1,94 & 1,$48 ; 4,1$ & P98; M97 \\
$i(\mathrm{gr})$. & $78^{\circ}$ & $78^{\circ}$ & $78^{\circ}$ & $\mathrm{C} 00$ \\
$\mathrm{M}_{\text {ion }} / \mathrm{M} \odot$ & 0,013 & & - & - \\
\hline
\end{tabular}

P98:Phillips (1998); MK96:McKenna et al. (1996); PM91:Preite-Martinez et al. (1991);

Z93:Zhang e Kwok (1993); M97:Mal'Kov (1997); T92:Tylenda et al. (1992);

A92:Acker et al. (1992); C00:Corradi et al. (2000)

Com base nos resultados dos modelos, que não apresentaram uma ampla descrição de todas as características observadas, espera-se que o cenário das propriedades geométricas de IC2553 seja mais complexo que os simulados. Isto é reforçado pelo seu imageamento, mesmo não sendo de grande resolução angular, que indica a presença de dupla camada de matéria ou uma variação drástica da mesma.

É interessante notar que, a exemplo do que as observações indicam para NGC5882, a presença da sub-estrutura na região equatorial de IC2553 é pronunciada pelas emissões de 
Tabela 4.16 - Propriedades geométricas do modelo LD (limitado por densidade) de IC2553.

\begin{tabular}{ccc}
\hline \hline Distância da fonte central & Formato & Valores \\
\hline Função do ângulo polar $\theta$ & $R_{0} / \cos \theta$ & $R_{0}: 8 \mathrm{e} 16 \mathrm{~cm} ; \theta_{\max }: 68^{\circ}$ \\
\hline & & \\
\hline Densidades & Formato & Valores \\
\hline Distribuição angular & dfact $\times($ Dayal et al., 2000) & dfact: 0,6 \\
Variação radial & constante em espessura $\Delta R$ & $\Delta R: 2 \mathrm{e} 16 \mathrm{~cm}$ \\
\hline
\end{tabular}

Tabela 4.17 - Propriedades geométricas do modelo LI (limitado por ionização) de IC2553.

\begin{tabular}{ccc}
\hline \hline Distância da fonte central & Formato & Valores \\
\hline Função do ângulo polar $\theta$ & $r_{0} / \cos \theta$ & $r_{0}: 2,1 \mathrm{e} 16 \mathrm{~cm} ; \theta_{\max }: 63,5^{\circ}$ \\
\hline & & \\
\hline Densidades & Formato & Valores \\
\hline Distribuição angular & dfact $\times($ Dayal et al., 2000$)$ & dfact: 0,625 \\
Variação radial & nula & - \\
\hline
\end{tabular}

Tabela 4.18 - Valores de $\chi^{2}$ entre os modelos finais e as observações de IC2553.

\begin{tabular}{llllll}
\hline \hline Mapas & $\chi_{\text {perp }}^{2 L I}$ & $\chi_{\text {para }}^{2 L I}$ & $\chi_{\text {perp }}^{2 L D}$ & $\chi_{\text {para }}^{2 L D}$ & $\sigma$ típico \\
\hline $\mathrm{n}_{e}$ & 47,2 & 49,3 & 33,0 & 41,1 & 1500 \\
$(\lambda 6548+6584) / \mathrm{H} \alpha$ & $(3157)$ & $(2524)$ & 324,7 & 774,7 & 0,12 \\
$\log [\lambda 6312 /(\lambda 6716+6731)]$ & 50,6 & 240,8 & 35,4 & 79,1 & $-0,08$ \\
$\log [(\lambda 6716+6731) / \lambda 6312]$ & 7,1 & 27,8 & 8,4 & 13,4 & 0,15 \\
\hline
\end{tabular}

[SII] em relação a [SIII], mas não possui variação mensurável nas densidades eletrônicas obtidas através das linhas de [SII]. Uma possível explicação para este fenômeno seria sua ocorrência em regiões mais internas (ou eventualmente externas) da nuvem, sem uma sensível mudança de densidades entre a sub-estrutura e o restante da nuvem). 

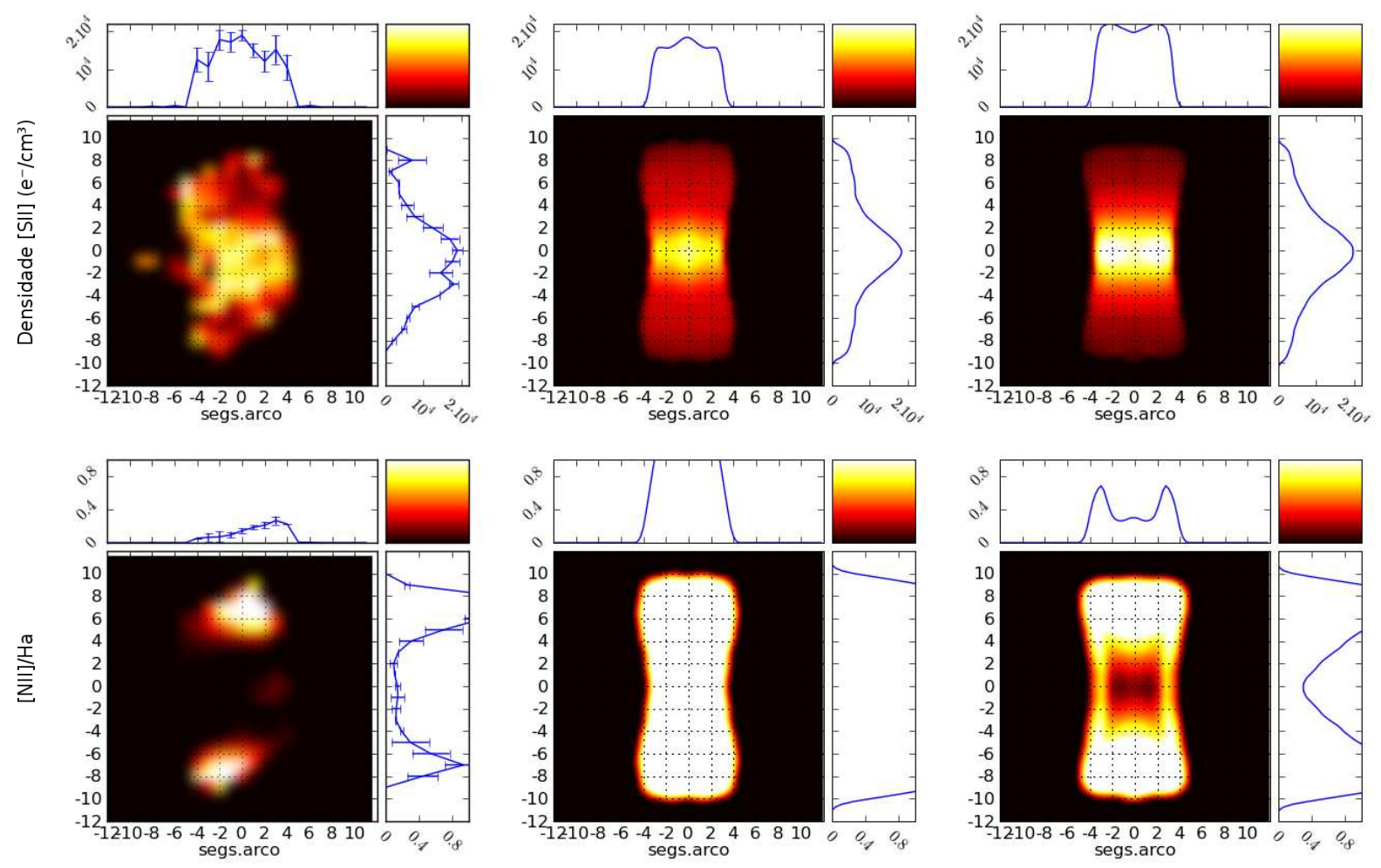

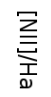
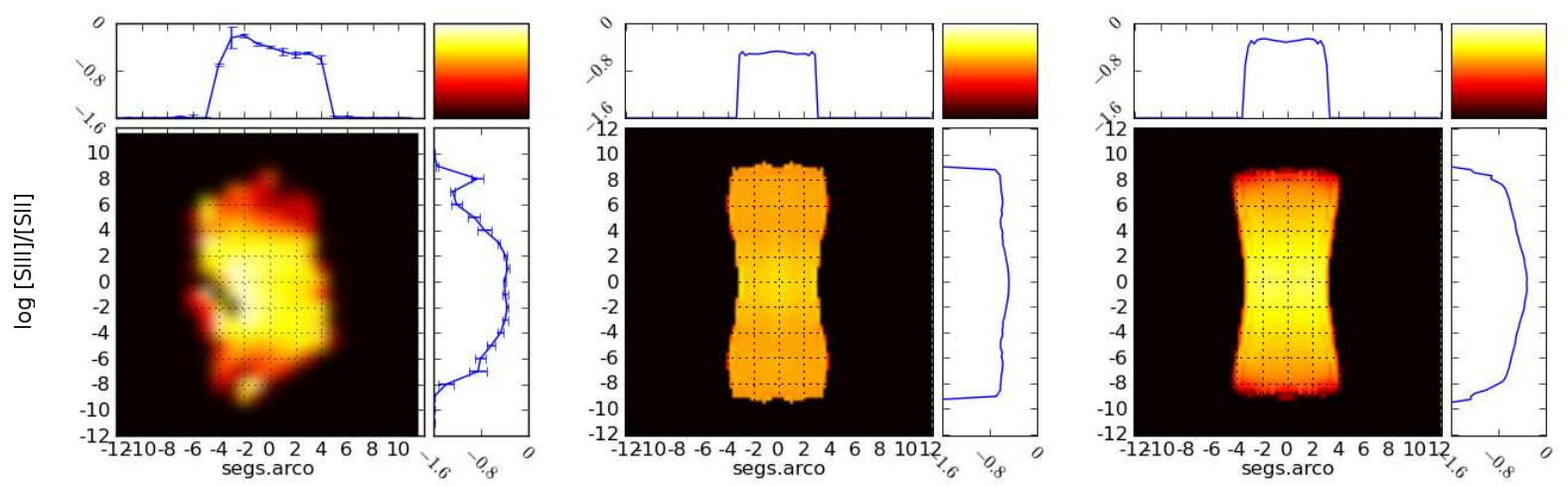

$\bar{\Xi}$
$\stackrel{\underline{\underline{\Xi}}}{\bar{v}}$
$\underline{\underline{\underline{v}}}$
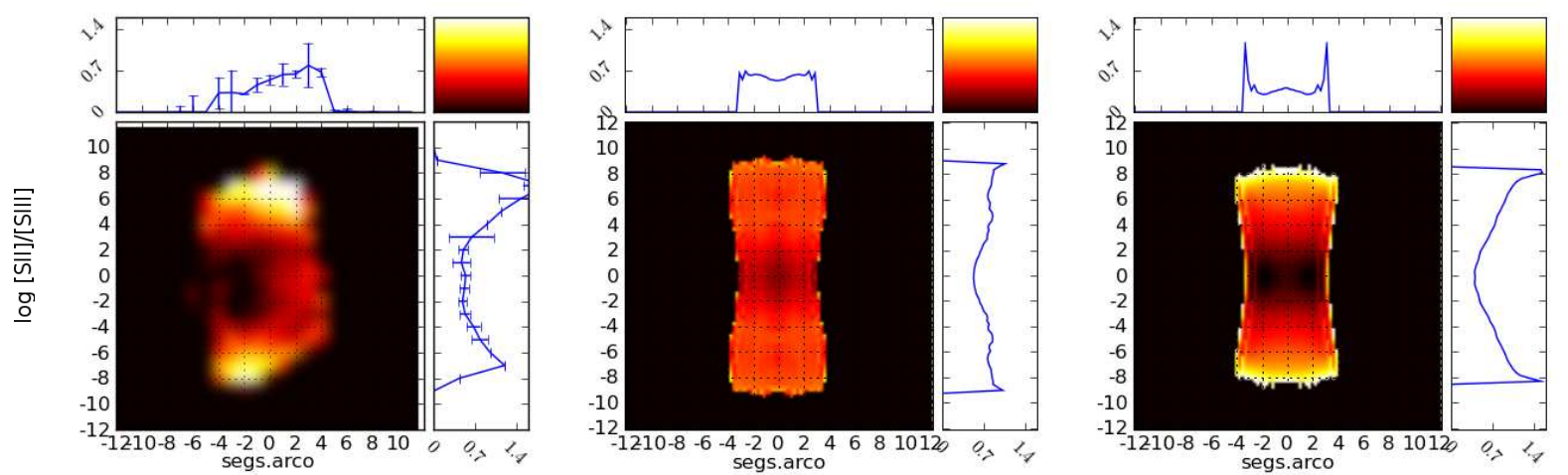

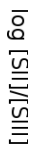

Figura 4.14: Comparação entre os dados observacionais de IC2553 (à esquerda) e as respectivas previsões de modelos. Ao centro, melhor modelo vinculando-se o fluxo $\mathrm{H} \beta$ observado (limitado por densidade). À direita, o modelo de melhor ajuste de $\chi^{2}$ (limitado por ionização). 


\subsubsection{NGC5882}

Este é um objeto para o qual existem imagens de alta resolução do telescópio espacial Hubble. Destaca-se a presença de dois envoltórios distintos neste objeto, além da presença de subestruturas irregulares (figura 4.15, (Hajian et al., 2007)).

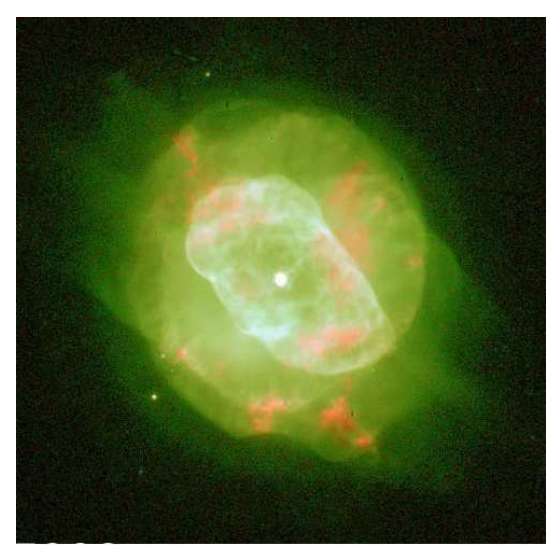

Figura 4.15: Imageamento de NGC5882 feita pelo telescópio espacial Hubble (WFPC2). Cores $\mathrm{R}: \mathrm{G}: \mathrm{B}=\log [\mathrm{NII}]: \log [\mathrm{OIII}]: \mathrm{V}$ linear. A orientação é arbitrária.

Através de considerações cinemáticas, no trabalho de Corradi et al. (2000) foi determinado o ângulo de projeção como $i=80^{\circ}$ para este objeto.

Utilizando a hipótese de dupla camada sugerida pelo imageamento, as simulações iniciais partiram da descrição de camada interna por um pico gaussiano e mantida a segunda camada de matéria constante, na hipótese NP limitada por densidade. Os resultados levaram a valores discrepantes tanto do fluxo $\mathrm{H} \beta$ total, quanto dos mapas [NII] $/ \mathrm{H} \alpha$, apresentando sistematicamente maior fluxo de linhas [NII].

A geometria das camadas foi determinada como sendo a camada interna um elipsóide de razão entre eixos de 1,8, enquanto a camada externa como um elipsóide de razão 1,2.

Buscou-se então a construção de modelos com a variação radial determinada através de dois picos gaussianos, na hipótese de NP limitada por densidade (ver equação na tabela 4.22). Um valor mínimo de $50 e^{-} / \mathrm{cm}^{3}$ foi mantido como constante na extensão da nuvem, afim de se evitar "vazios" entre os picos (e consequentemente a física de baixas densidades nas simulações, que consomem maior esforço computacional no programa Cloudy). Este valor constante de densidade também melhora o ajuste tridimensional entre os fluxos obtidos a cada uma das direções polares simuladas, sendo o valor típico em NPs de baixa 
Tabela 4.19 - Parâmetros do modelo final de NGC5882 comparados com referências.

\begin{tabular}{|c|c|c|c|}
\hline Parâmetro & Modelo & Valores de Ref. & Referências \\
\hline $\bar{n}_{e}\left(e^{-} / c m^{3}\right)$ & 10000 & 4000 & T03 \\
\hline $\bar{T}_{[N I I]}(\mathrm{K})$ & 9190 & $7720 ; 10800$ & MK96; T03 \\
\hline $\bar{T}_{[O I I I]}(\mathrm{K})$ & - & $8670 ; 9400$ & MK96; T03 \\
\hline $10^{3} \cdot T_{e f f}(\mathrm{~K})$ & 60000 & 66,$5 ; 73 ; 59,6$ & PM89; Z93; M97 \\
\hline $\mathrm{L} / \mathrm{L}_{\odot}$ & 6310 & $6200 ; 6300$ & Z93; M97 \\
\hline Espectro & $\mathrm{H}-\mathrm{Ni}$ & $\mathrm{Of}(\mathrm{H})$ & A92 \\
\hline $\log g\left(\mathrm{~cm} / \mathrm{s}^{2}\right)$ & 5,2 & 4,$5 ; 4,8$ & Z93; M97 \\
\hline $\bar{E}(B-V)$ & 0,38 & 0,38 & T92 \\
\hline $\mathrm{F}_{H \beta}^{t o t a l}(\mathrm{erg} / \mathrm{cm} . \mathrm{s})$ & $8,94 \mathrm{E}-011$ & $1,02 \mathrm{E}-10$ & A92 \\
\hline $\mathrm{d}(\mathrm{kpc})$ & 0,76 & 2,7 & M97 \\
\hline $\mathrm{M}_{i o n} / \mathrm{M}_{\odot}$ & 0,01 & & \\
\hline \multicolumn{4}{|c|}{ T03:(Tsamis et al., 2003); MK96:McKenna et al. (1996); PM89:Preite-Martinez et al. (1989); } \\
\hline Z93:Zhang e Kwok (1 & 93); M97:Mal' & ov (1997); T92:Tylen & a et al. (1992); \\
\hline A92:Acker & & & \\
\hline
\end{tabular}

densidade (Gurzadyan, 1997).

Assim obtiveram-se bons resultados com a hipótese de NP limitada por ionização. O melhor modelo, apresentado na tabela 4.19, foi conseguido coincidindo a região polar da camada interna com a camada externa. A composição química utilizada está descrita na tabela 4.20 (Pottasch e Bernard-Salas, 2006). Destacamos que a presença de emissão em forma de halo é observada para este objeto (Schwarz et al., 1992) e pode ser um indicativo da limitação por ionização do objeto (ver discussão na seção 5).

Uma característica dos mapas observacionais de ionização do Enxofre (S) é a variação dos fluxos nas extremidades inferior e superior dos mapas. Nestas regiões, há uma predominância de emissão [SII] em comparação com a emissão [SIII]. Tal característica reflete a distribuição de matéria na região polar, com a sobreposição das camadas interna e externa e maior distância à fonte central e são previstas pelo modelo. Apesar das características diferenciadas desta região, somente uma pequena diferença na densidade projetada é es- 
Tabela 4.20 - Composição química utilizada para NGC5882 nas simulações.

\begin{tabular}{cc}
\hline \hline Parâmetro & Valor adotado \\
\hline $\mathrm{He} / \mathrm{H}$ & 0,108 \\
$\epsilon(\mathrm{O})$ & 8,68 \\
$\epsilon(\mathrm{N})$ & 8,20 \\
$\epsilon(\mathrm{S})$ & 7,11 \\
$\epsilon(\mathrm{Ne})$ & 8,18 \\
$\epsilon(\mathrm{Ar})$ & 6,46 \\
\hline
\end{tabular}

Tabela 4.21 - Valores de $\chi^{2}$ entre o modelo final e as observações de NGC5882.

\begin{tabular}{cccc}
\hline \hline Mapas & $\chi_{\text {perp }}^{2}$ & $\chi_{\text {para }}^{2}$ & Erro típico \\
\hline $\mathrm{n}_{e}$ & 26,1 & 23,6 & 1500 \\
$\log [\lambda 6312 /(\lambda 6716+6731)]$ & 104,0 & 144,7 & $-0,1$ \\
$\log [(\lambda 6716+6731) / \lambda 6312]$ & 25,3 & 19,8 & 0,2 \\
\hline
\end{tabular}

perada.

Outra característica dos mapas de ionização de $\mathrm{S}$ é a não-homogeneidade da região equatorial, em oposição ao valor constante esperado pelo modelo. Na região apresentada à esquerda, nota-se alto valor de emissão [SII] e baixa emissão [SIII], exatamente o contrário do que ocorre na região à direita. Comparando tais regiões com o imageamento de altaresolução, nota-se a aproximada sobreposição de uma subestrutura à esquerda (brilhante em [NII]) e de uma região densa em brilho à direita. Assim, uma influência da subestrutura irregular é verificada, em conformidade com outras observações que prevêem tais estruturas como de baixa ionização ( "low ionization structures", em inglês). 

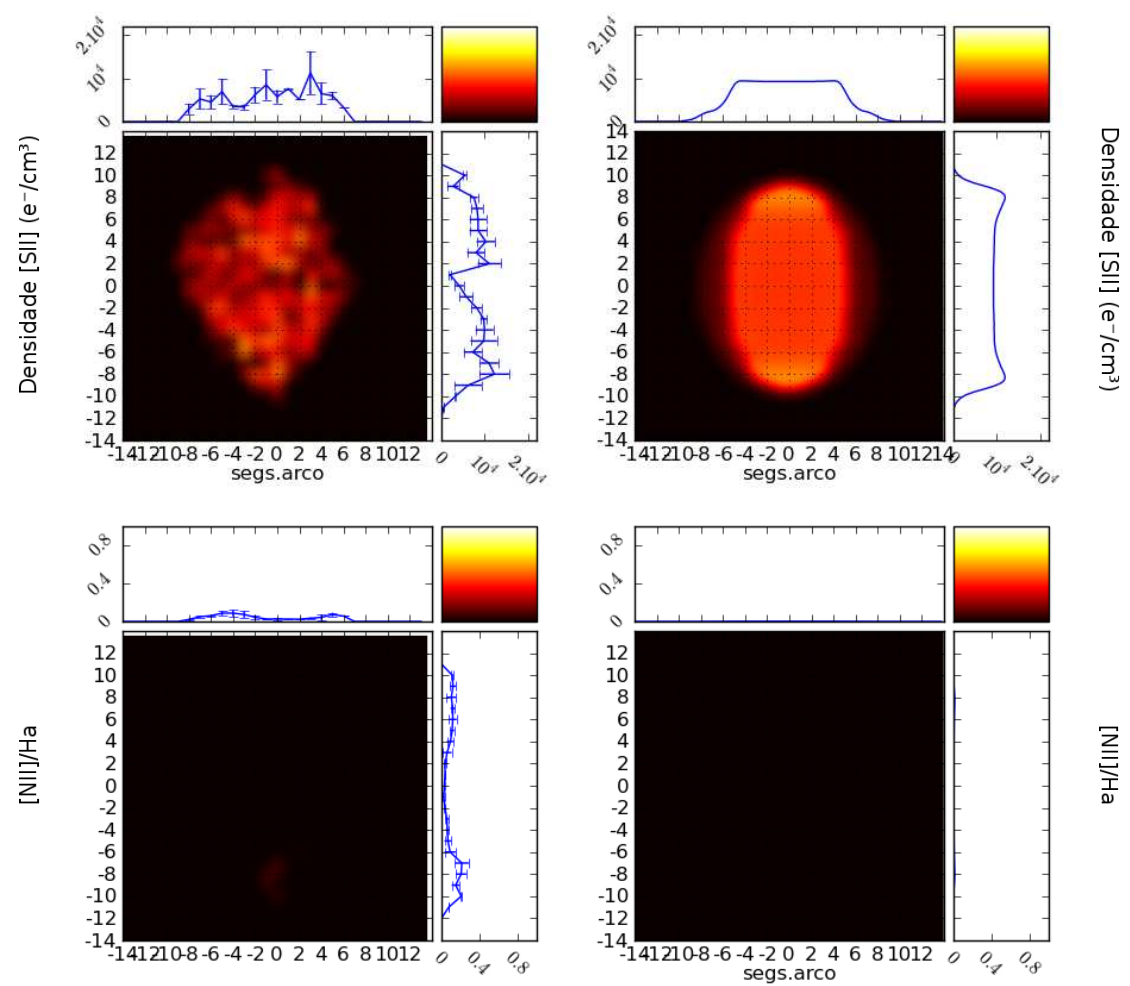

$\underset{\substack{\text { I } \\ \text { I }}}{\stackrel{\sum}{T}}$
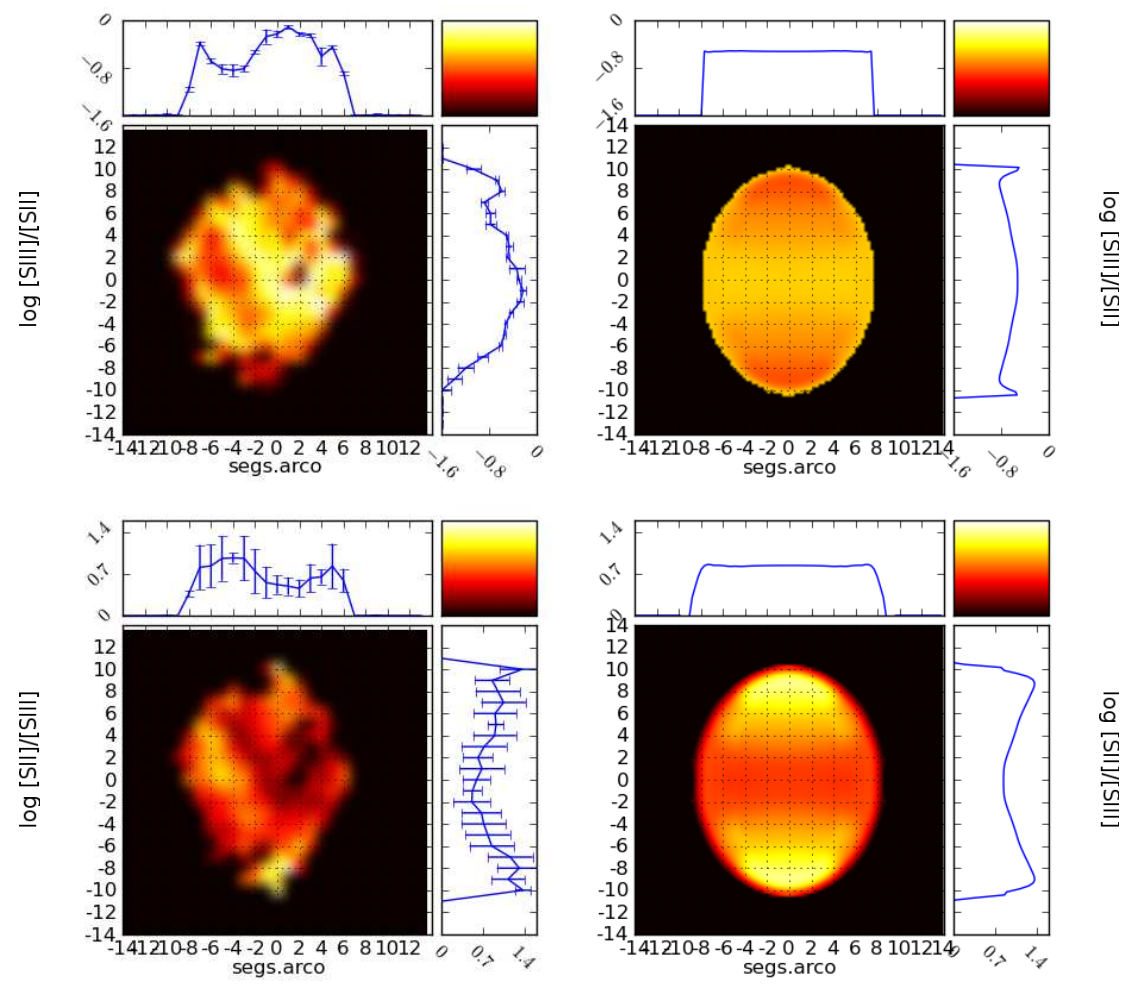

Figura 4.16: Comparação entre os dados observacionais de NGC5882 (à esquerda) e as respectivas previsões do modelo (à direita). 
Tabela 4.22 - Propriedades geométricas do modelo de NGC5882.

\begin{tabular}{ccc}
\hline \hline Distância da fonte central & Formato & Valores \\
\hline Em função do ângulo polar $\theta$ & $R_{0} \times \frac{a_{0}}{b_{0}} \frac{1}{\sqrt{(b \sin \theta)^{2}+(a \cos \theta)^{2}}}$ & $R_{0}: 5 \mathrm{e} 15 \mathrm{~cm} ; a_{0} / b_{0}: 1,8$ \\
\hline Densidades & Formato & Valores \\
\hline Distribuição angular & constante & $n_{0}: 10000 \mathrm{H} / \mathrm{cm}^{3} ; \sigma_{0}: 2,7 \mathrm{e} 15 \mathrm{~cm} ;$ \\
Variação radial $\Delta R$ & $n_{c}+n_{0} \times \exp ^{-\left(\frac{\Delta R-3 \sigma_{0}}{\sigma_{0}}\right)^{2}}+$ & $n_{1}: 1000 \mathrm{H} / \mathrm{cm}^{3} ; \sigma_{1}: 6,7 \mathrm{e} 15 \mathrm{~cm}$ \\
& $+n_{1} \times \exp ^{-\left(\frac{\Delta R-3 \sigma_{1}+f\left(\theta_{1}, a_{1}\right)}{\sigma_{1}}\right)^{2}}$ & $f\left(\theta_{1}, a_{1}\right):$ ver texto; \\
\end{tabular}




\subsubsection{IC1297}

Uma imagem do objeto encontra-se na figura 4.17, de R. Corradi (Schwarz et al., 1992).

Dentre a amostra de objetos observados com espectroscopia de campo integral, este é o objeto que possui dados observacionais que mais se distanciam da simetria de bipolar. Enquanto que a maioria dos objetos apresenta uma região central com característica distinta das regiões polares (por exemplo, no caso da densidade [SII], maior contraste de densidade de elétrons na região central) ou certa uniformidade, neste objeto pode-se delinear somente um pólo com características distintas da região complementar.

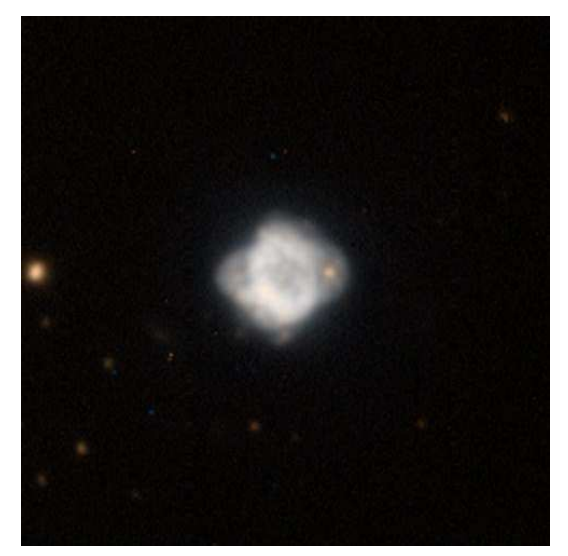

Figura 4.17: Imageamento de IC1297. Cores R:G:B $=\log ([\mathrm{NII}]+\mathrm{H} \alpha)$ :ambos:log[OIII]. Orientação arbitrária.

Assim, para a geometria do objeto simulou-se inicialmente a geometria de elipsóide, dada certa proximidade de valores com o objeto NGC5882. Uma geometria deste tipo sob a condição de casca de densidade variável é capaz de gerar mapas compatíveis com a ausência de características circulares/elípticas para o objeto, pronunciáveis outros objetos de geometria elíptica, como NGC6572 e IC4776.

Entretanto, tais modelos previam sistematicamente um fluxo $\mathrm{H} \beta$ total para o objeto e/ou um fluxo [NII] maior do que o observado.

A configuração do tipo tubo apresentou resultados mais consistentes com os vínculos e ajuste de melhor $\chi^{2}$. Como para IC2553, distintos envoltórios de densidade constante foram modelados como frações das distribuições de Dayal et al. (2000), além de alguns modelos de densidade constante mas limitados por densidade. As características do modelo de melhor ajuste $\chi^{2}$ em compatibilidade outras grandes observacionais está descrito na tabela 4.23. 


\begin{tabular}{ccc}
\hline \hline Distância da fonte central & Formato & Valores \\
\hline Em função do ângulo polar $\theta$ & $R_{0} / \cos \theta$ & $R_{0}: 2 \mathrm{e} 16 \mathrm{~cm} ; \theta_{\max }: 60^{\circ}$ \\
\hline & & \\
\hline Densidades & Formato & Valores \\
\hline Distribuição angular & dfact $\times($ Dayal et al., 2000) & dfact: 0,35 \\
Variação radial & constante em espessura $\Delta R$ & $\Delta R: 2 \mathrm{e} 16 \mathrm{~cm}$ \\
\hline
\end{tabular}

Tabela 4.23 - Propriedades geométricas do modelo de IC1297.

$\mathrm{Na}$ ausência de informações sob a projeção do objeto na esfera celeste, o ângulo de projeção $i$ foi variado entre $20^{\circ}$ e $90^{\circ}$. O que mostrou melhor correspondência dos dados foi $i=45^{\circ}$. Uma definição da geometria de IC1297 poderia ser melhor definida com um estudo cinemático do objeto.

O valor da composição química adotada nas simulações está na tabela 4.24, obtida de Girard et al. (2007). As características do modelo final estão na tabela 4.25, com a respectiva comparação de parâmetros com referências. Os valores de ajuste $\chi^{2}$ estão na tabela 4.26 .

Tabela 4.24 - Valores de composição química de IC1297 adotados para as simulações.

\begin{tabular}{cc}
\hline \hline Parâmetro & Valor adotado \\
\hline $\mathrm{He} / \mathrm{H}$ & 0,112 \\
$\epsilon(\mathrm{O})$ & 8,77 \\
$\epsilon(\mathrm{N})$ & 8,37 \\
$\epsilon(\mathrm{S})$ & 7,10 \\
$\epsilon(\mathrm{Ne})$ & 8,10 \\
$\epsilon(\mathrm{Ar})$ & 6,27 \\
\hline
\end{tabular}

A presença de subestruturas pode estar relacionada com a origem da assimetria neste objeto, como indica a particularidade dos fluxos registrados na região superior dos mapas em comparação com outros objetos observados (NGC5882 e IC2553). 
Tabela 4.25 - Parâmetros do modelo final de IC1297 comparados com referências.

\begin{tabular}{cccc}
\hline \hline Parâmetro & Modelo & Valores de Ref. & Referências \\
\hline $\bar{n}_{e}\left(e^{-} / \mathrm{cm}^{3}\right)$ & 7000 & 2800 & $\mathrm{G} 07$ \\
$\bar{T}_{[N I I]}(\mathrm{K})$ & 10625 & 10300 & $\mathrm{G} 07$ \\
$\bar{T}_{[\text {OIII }}(\mathrm{K})$ & - & 10100 & $\mathrm{G} 07$ \\
$10^{3} . T_{\text {eff }}(\mathrm{K})$ & 88 & 88,$8 ; 93,2$ & $\mathrm{PM} 89 ; \mathrm{M} 97$ \\
$\mathrm{~L} / \mathrm{L} \odot$ & 5600 & 5600 & $\mathrm{M} 97$ \\
$\mathrm{Espectro}$ & $\mathrm{PG} 1159$ & $\mathrm{WC} 4 ; \mathrm{WO} 3$ & $\mathrm{~A} 92 ; \mathrm{G} 07$ \\
$\log \mathrm{g}\left(\mathrm{cm} / \mathrm{s}^{2}\right)$ & 5,3 & 5,3 & \\
$\bar{E}(B-V)$ & 0,19 & 0,$19 ; 0,18$ & $\mathrm{~T} 92 ; \mathrm{G} 07$ \\
$\mathrm{~F}_{H \beta}^{\text {total }}(\mathrm{erg} / \mathrm{cm} . \mathrm{s})$ & $1,77 \mathrm{E}-011$ & $1,74 \mathrm{E}-011$ & $\mathrm{~A} 92$ \\
$\mathrm{~d}(\mathrm{kpc})$ & 0,66 & 2,4 & $\mathrm{M} 97$ \\
$i(\mathrm{gr})$. & $45^{\circ}$ & - & - \\
$\mathrm{M}_{\text {ion }} / \mathrm{M}_{\odot}$ & 0,001 & - & - \\
\hline
\end{tabular}

G07:Girard et al. (2007); PM89:Preite-Martinez et al. (1989); M97:Mal'Kov (1997);

A92:Acker et al. (1992); T92:Tylenda et al. (1992)

Tabela 4.26 - Valores de $\chi^{2}$ entre o modelo final e as observações de NGC1297.

\begin{tabular}{cccc}
\hline \hline Mapas & $\chi_{\text {perp }}^{2}$ & $\chi_{\text {para }}^{2}$ & Erro típico \\
\hline $\mathrm{n}_{e}$ & 29,8 & 91,4 & 1750 \\
$(\lambda 6548+6584) / \mathrm{H} \alpha$ & - & - & 0,05 \\
$\log [\lambda 6312 /(\lambda 6716+6731)]$ & 66,5 & 84,4 & $-0,08$ \\
$\log [(\lambda 6716+6731) / \lambda 6312]$ & 32,1 & 65,4 & 0,15 \\
\hline
\end{tabular}



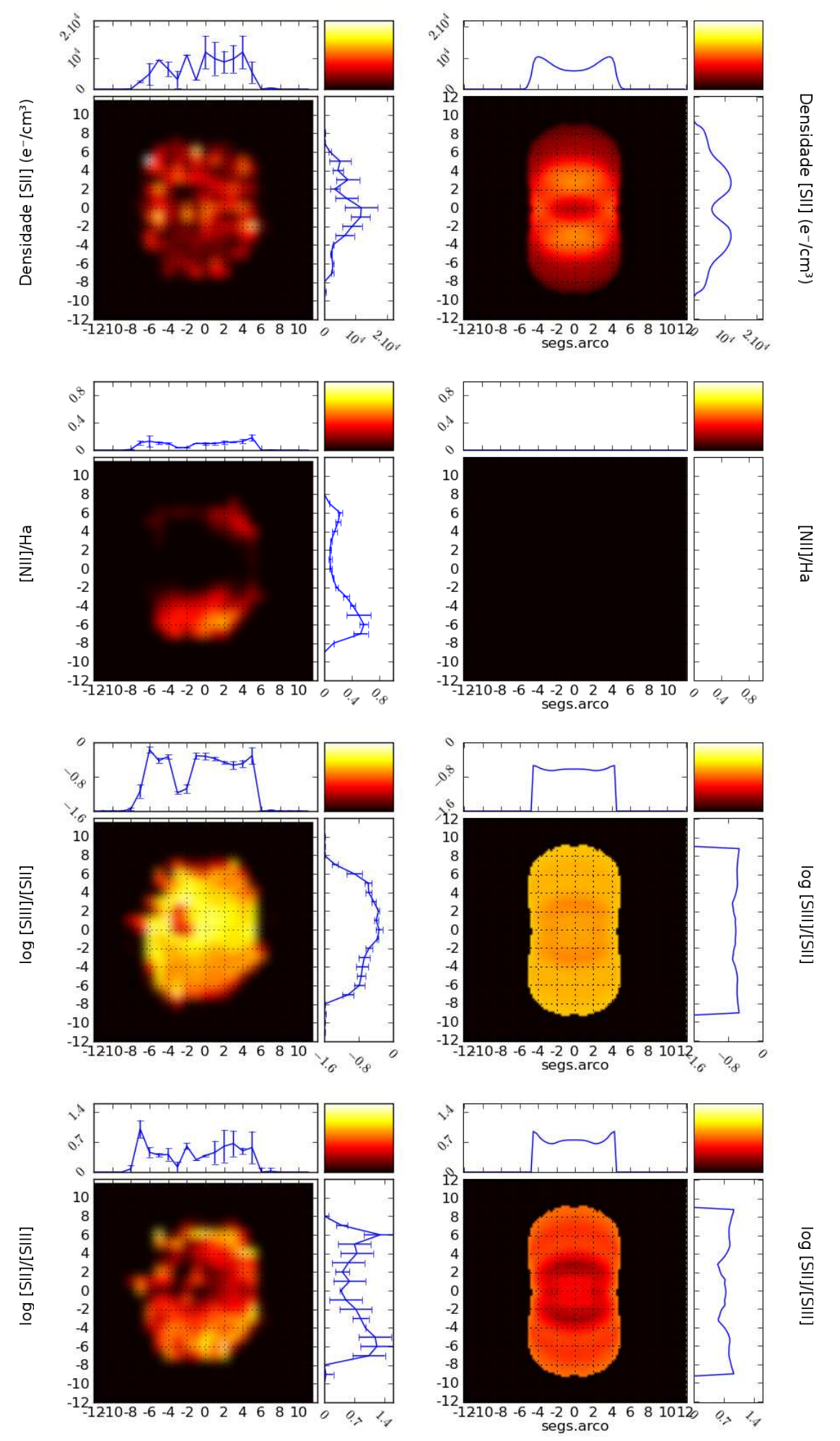

Figura 4.18: Comparação entre os dados observacionais de IC1297 (à esquerda) e as respectivas previsões do modelo (à direita). 


\section{$4.2 .5 \quad I C 4776$}

Uma imagem do objeto encontra-se na figura 4.19, de R. Corradi (Schwarz et al., 1992).

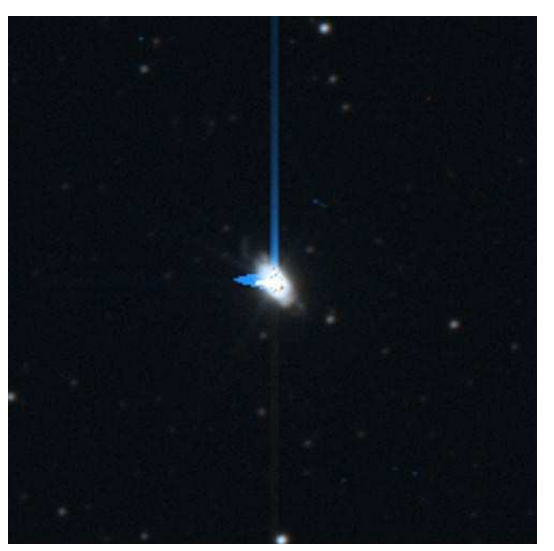

Figura 4.19: Imageamento de IC4776. Cores R:G:B $=\log ([\mathrm{NII}]+\mathrm{H} \alpha)$ :ambos: $\log [\mathrm{OIII}]$. Orientação arbitrária.

Observando os mapas de razão de fluxos determinados para este objeto, vemos sua similaridade com o caso de NGC6572 de geometria elipsoidal. Dada a ausência de informação sobre o ângulo de projeção do objeto, o formato elipsoidal foi proposto para este objeto, variando-se o ângulo de projeção do eixo maior do objeto com a linha de visada $i$ entre $45^{\circ}$ e $90^{\circ}$. Os valores de composição química utilizados para as simulações estão na tabela 4.27 (de Freitas Pacheco et al., 1992).

Tabela 4.27 - Composição química adotada para as simulações de IC4776.

\begin{tabular}{cr}
\hline \hline Parâmetro & Valor adotado \\
\hline $\mathrm{He} / \mathrm{H}$ & 0,090 \\
$\epsilon(\mathrm{O})$ & 8,90 \\
$\epsilon(\mathrm{N})$ & 7,78 \\
$\epsilon(\mathrm{S})$ & 6,94 \\
$\epsilon(\mathrm{Ne})$ & 7,98 \\
$\epsilon(\mathrm{Ar})$ & 6,47 \\
\hline
\end{tabular}


Tabela 4.28 - Parâmetros dos modelos de IC4776, comparado com referências.

\begin{tabular}{ccccc}
\hline \hline Parâmetro & Modelo LD & Modelo LI & Valores de Ref. & Referências \\
\hline $\bar{n}_{e}\left(e^{-} / \mathrm{cm}^{3}\right)$ & 9400 & 10000 & 30000 & FP92 \\
$\bar{T}_{[N I I]}(\mathrm{K})$ & 8426 & 9412 & 9000 & FP92 \\
$\bar{T}_{[\text {OIII }]}(\mathrm{K})$ & - & - & 9000 & FP92 \\
$10^{3} \cdot T_{e f f}(\mathrm{~K})$ & 75 & 60 & 60 & HB04 \\
$\mathrm{L} / \mathrm{L} \odot$ & 1585 & 1585 & 1600 & HB04 \\
Espectro & $\mathrm{PG} 1159$ & $\mathrm{PG} 1159$ & $\mathrm{WC} 6$ & $\mathrm{~A} 92$ \\
$\log \mathrm{g}\left(\mathrm{cm} / \mathrm{s}^{2}\right)$ & 5,1 & 5,1 & 5,1 & $\mathrm{HB} 04$ \\
$\bar{E}(B-V)$ & 0,2 & 0,2 & 0,$2 ; 0,14$ & T92; FP92 \\
$\mathrm{F}_{H \beta}^{\text {total }}(\mathrm{erg} / \mathrm{cm} . \mathrm{s})$ & $3,32 \mathrm{E}-011$ & $2,42 \mathrm{E}-010$ & $3,02 \mathrm{E}-011$ & $\mathrm{~A} 92$ \\
$\mathrm{~d}(\mathrm{kpc})$ & 1,36 & 1,46 & - & - \\
$i(\mathrm{gr})$. & $90^{\circ}$ & $90^{\circ}$ & - & - \\
$\mathrm{M}_{\text {ion }} / \mathrm{M}_{\odot}$ & 0,008 & 0,04 & - & - \\
\hline
\end{tabular}

FP92:de Freitas Pacheco et al. (1992); HB04:Herald e Bianchi (2004); T92:Tylenda et al. (1992)

A92:Acker et al. (1992)

Tabela 4.29 - Valores de $\chi^{2}$ entre os modelos finais e as observações de IC4776.

\begin{tabular}{cccccc}
\hline \hline Mapas & $\chi_{\text {perp }}^{2(L D)}$ & $\chi_{\text {para }}^{2(L D)}$ & $\chi_{\text {perp }}^{2(L I)}$ & $\chi_{\text {para }}^{2(L I)}$ & Erro típico \\
\hline $\mathrm{n}_{e}$ & 127,8 & 198,7 & 94,3 & 130,0 & 1250 \\
$(\lambda 6548+6584) / \mathrm{H} \alpha$ & - & - & 117,54 & 111,3 & 0,05 \\
$\log [\lambda 6312 /(\lambda 6716+6731)]$ & 17,3 & 49,3 & 45,2 & 76,3 & 0,2 \\
$\log [(\lambda 6716+6731) / \lambda 6312]$ & 74,5 & 65,9 & 20,6 & 25,1 & $-0,1$ \\
\hline
\end{tabular}


Tabela 4.30 - Propriedades geométricas do modelo limitado por densidade (LD) de IC4776.

\begin{tabular}{ccc}
\hline \hline Distância da fonte central & Formato & Valores \\
\hline Em função do ângulo polar $\theta$ & $R_{0} \times \frac{a}{b} \frac{1}{\sqrt{(b \sin \theta)^{2}+(a \cos \theta)^{2}}}$ & $R_{0}: 8 \mathrm{e} 16 \mathrm{~cm} ; a / b: 1,43$ \\
\hline Densidades & Formato & Valores \\
\hline Distribuição angular & constante & $n_{0}: 7500 \mathrm{H} / \mathrm{cm}^{3} ; \sigma_{0}: 6,7 \mathrm{e} 15 \mathrm{~cm}$ \\
Variação radial $\Delta R$ & $n_{0} \times \exp ^{-\left(\left(\Delta R-3 \sigma_{0}\right) / \sigma_{0}\right)^{2}}$ & - \\
\hline
\end{tabular}

Tabela 4.31 - Propriedades geométricas do modelo limitado por ionização (LI) de IC4776.

\begin{tabular}{ccc}
\hline \hline Distância da fonte central & Formato & Valores \\
\hline Em função do ângulo polar $\theta$ & constante & $R_{0}: 1 \mathrm{e} 16 \mathrm{~cm}$ \\
\hline & & \\
\hline Densidades & Formato & Valores \\
\hline Distribuição angular & equação 3.2 & $m: 3 ; n_{0}: 5000 ; \epsilon: 3$ \\
Variação radial $\Delta R$ & nula & - \\
\hline
\end{tabular}



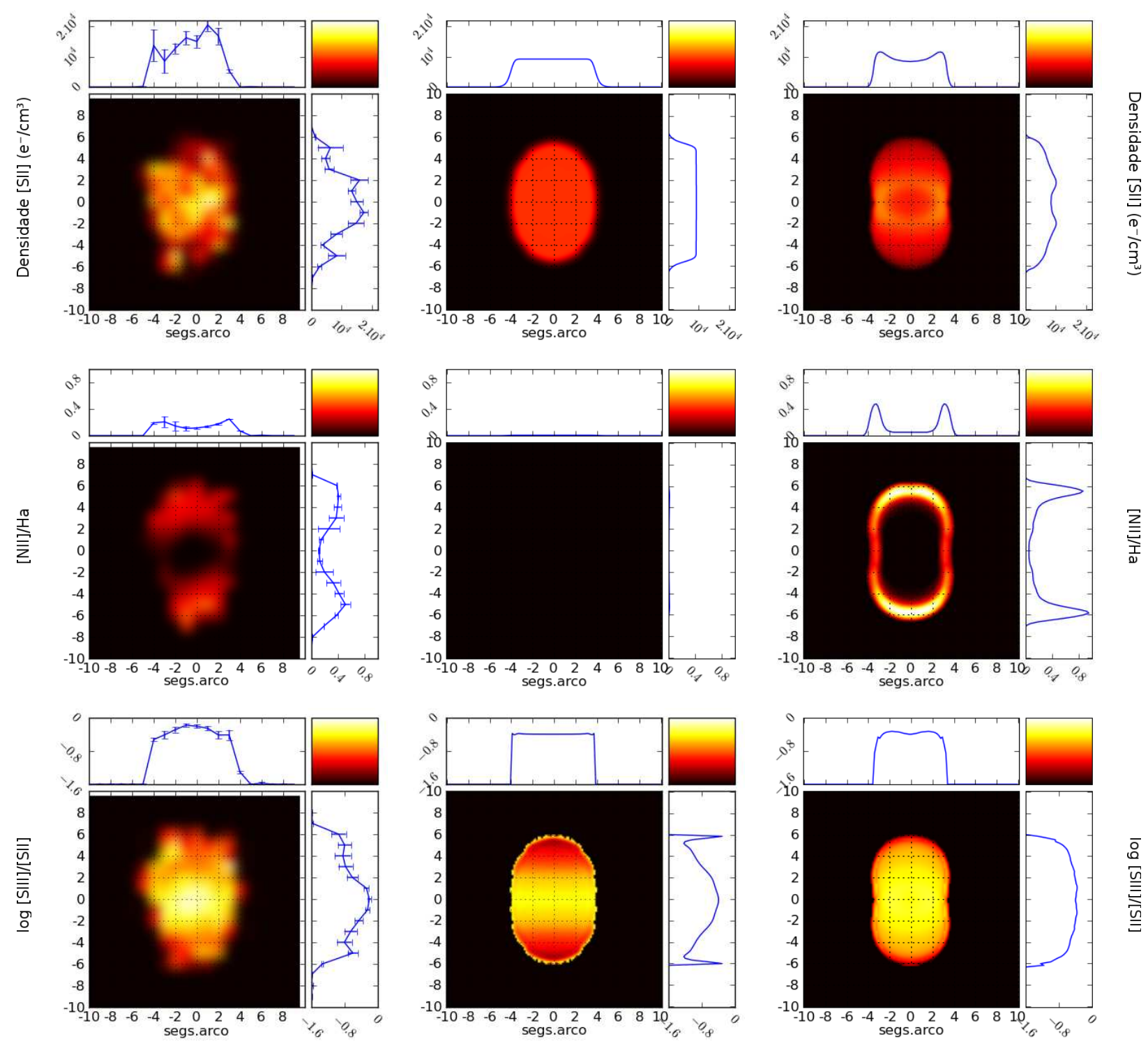

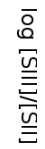
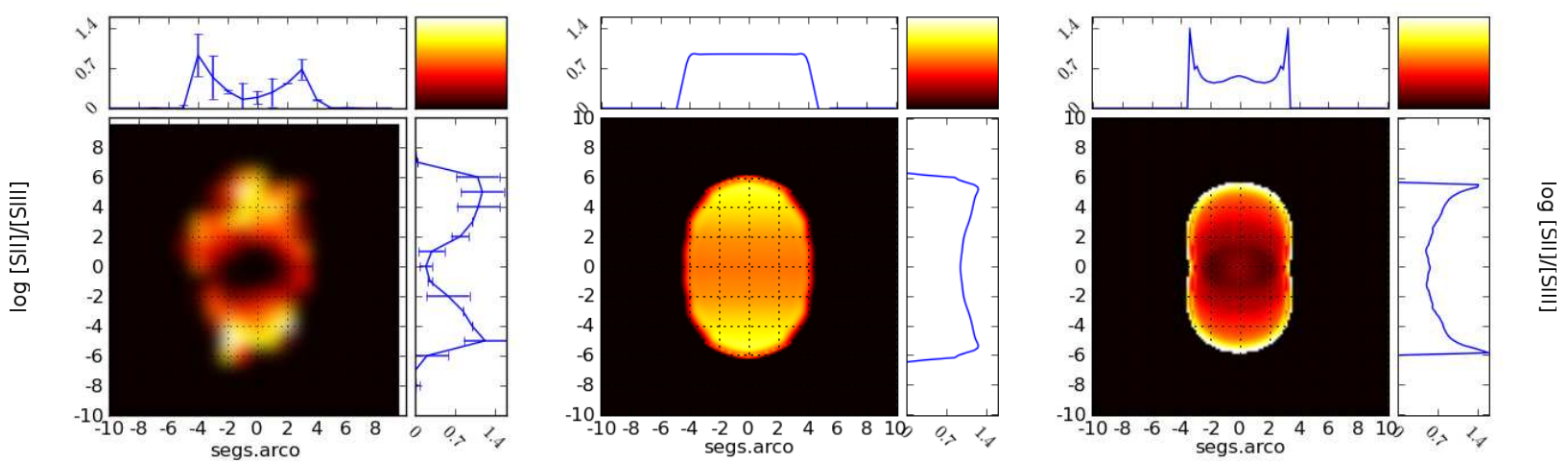

Figura 4.20: Comparação entre os dados observacionais de IC4776 (à esquerda) e as respectivas previsões de modelos. Ao centro, melhor modelo vinculando-se o fluxo $\mathrm{H} \beta$ observado (limitado por densidade). À direita, o modelo de melhor ajuste de $\chi^{2}$ (limitado por ionização). 


\subsection{Diagramas de diagnóstico}

O estudo da emissão das diferentes regiões das NPs nos diagramas de diagnóstico de plasma permitiu encontrar uma correlação entre o comportamento destas regiões e a qualidade com que se produziram os modelos. Sob espectroscopia de fenda longa, foram utilizados os fluxos das distintas janelas. Sob espectroscopia de campo integral, utilizou-se os fluxos provenientes do perfil dos objetos ao longo do eixo maior projetado.

Como resultado, a representação dos pontos das simulações nestes diagramas exibiu um comportamento regular. Nos diagramas $[S I I] \lambda 6716 / \lambda 6731 \times \log H \alpha /[N I I](\lambda 6548+6584)$ e $\times \log H \alpha /[S I I](\lambda 67125)$, os pontos dispersam-se entre o coeficiente angular esperado para as NPs de Sabbadin et al. (1977) até inclinações próximas a zero; no diagrama $[\log H \alpha /[S I I](\lambda 67125) \times \log H \alpha /[N I I](\lambda 6548+6584)$, o comportamento dá-se exatamente da forma forma prevista, mantendo-se basicamente a mesma relação angular.

As simulações são indicadas nos diagramas por círculos para os modelos limitados por ionização, e por quadrados para os modelos limitados por densidade. Em particular, para dois destes objetos (NGC6572 e IC4406), a representação dos pontos apresentou sistematicamente o mesmo comportamento linear das simulações, mesmo que levemente deslocados em um dos eixos. Os resultados são exibidos na figura 4.21. Os fluxos observados e modelados são comparados com os do catálogo Acker et al. (1992), que são denominados pela sigla A92.

Para NGC6572, a baixa razão das linhas $[S I I] \lambda 6716 / \lambda 6731$ reflete a alta densidade eletrônica registrada para este objeto.

Já para os outros objetos, distintos comportamentos são observados. Não só as razões de fluxos dispersam-se bem além das regiões esperadas, mas não se observa o padrão entre as regiões internas e externas da nuvem de acordo com as previsões de modelo. Nos diagramas $[S I I] \lambda 6716 / \lambda 6731 \times \log H \alpha /[N I I](\lambda 6548+6584)$ e $\times \log H \alpha /[S I I](\lambda 67125)$, os pontos dispersam-se muitas vezes verticalmente; no diagrama $[\log H \alpha /[S I I](\lambda 67125) \times$ $\log H \alpha /[N I I](\lambda 6548+6584)$, mantém-se em dispersão vertical.

Estes resultados observacionais indicam que as hipóteses utilizadas no trabalho para o cálculo dos fluxos não são capazes de descrever os comportamentos encontrados.

Em alguns destes objetos, o comportamento vertical no diagrama lembra a curva de remanescentes de supernovas, onde o mecanismo de choque é reconhecidamente intenso. 

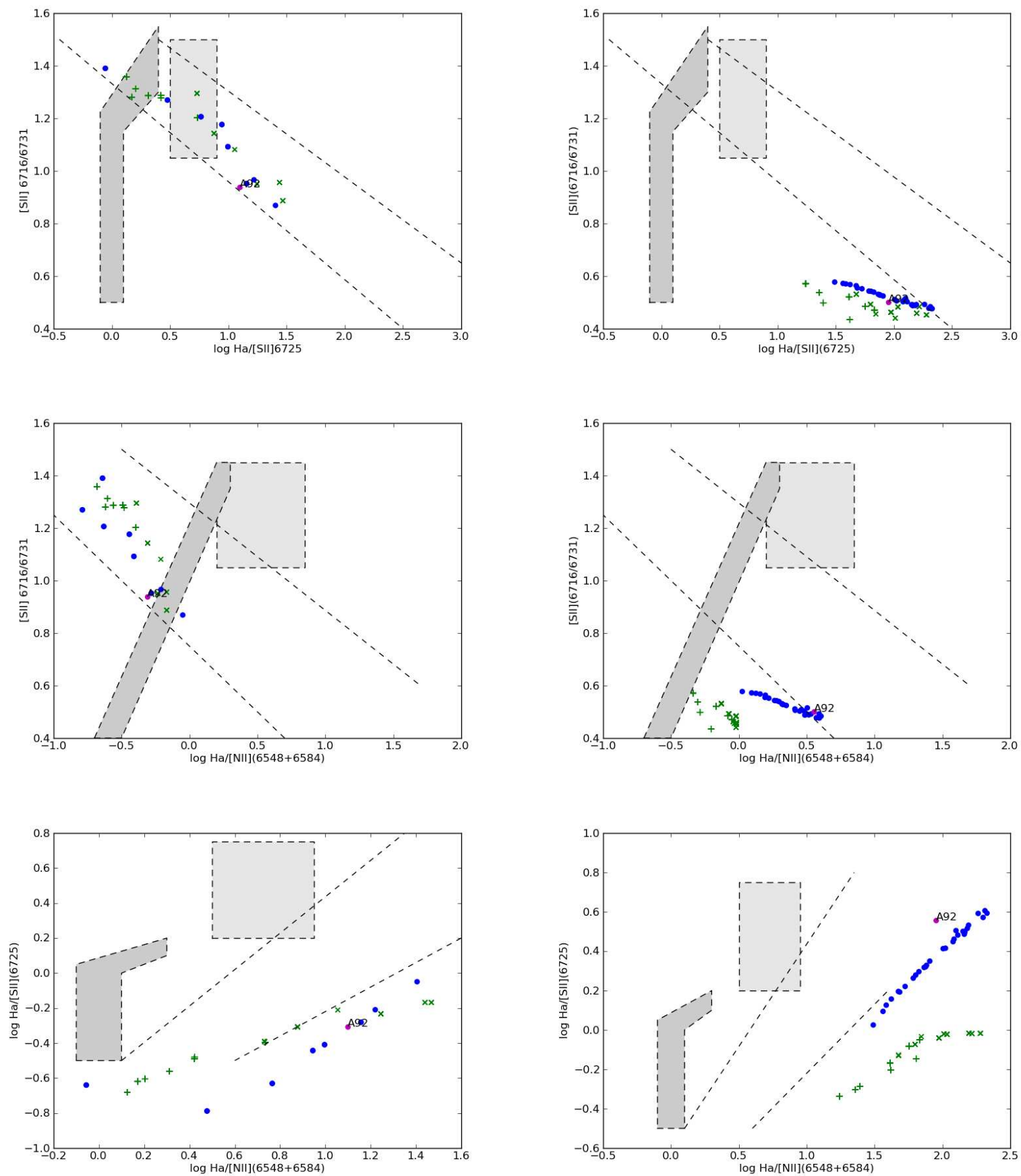

Figura 4.21: Diagramas de diagnóstico de plasma para IC4406 (à esquerda) e NGC6572 (à direita), em bom acordo com os modelos gerados. Em escuro, a região típica de remanescentes de supernova (RSN), em cinza claro a região de regiões HII, e entre as retas as regiões de NPs. Os pontos observacionais das NPs são exibidos como "+" (regiões externas) e "x" (regiões internas), comparados com os modelos. 

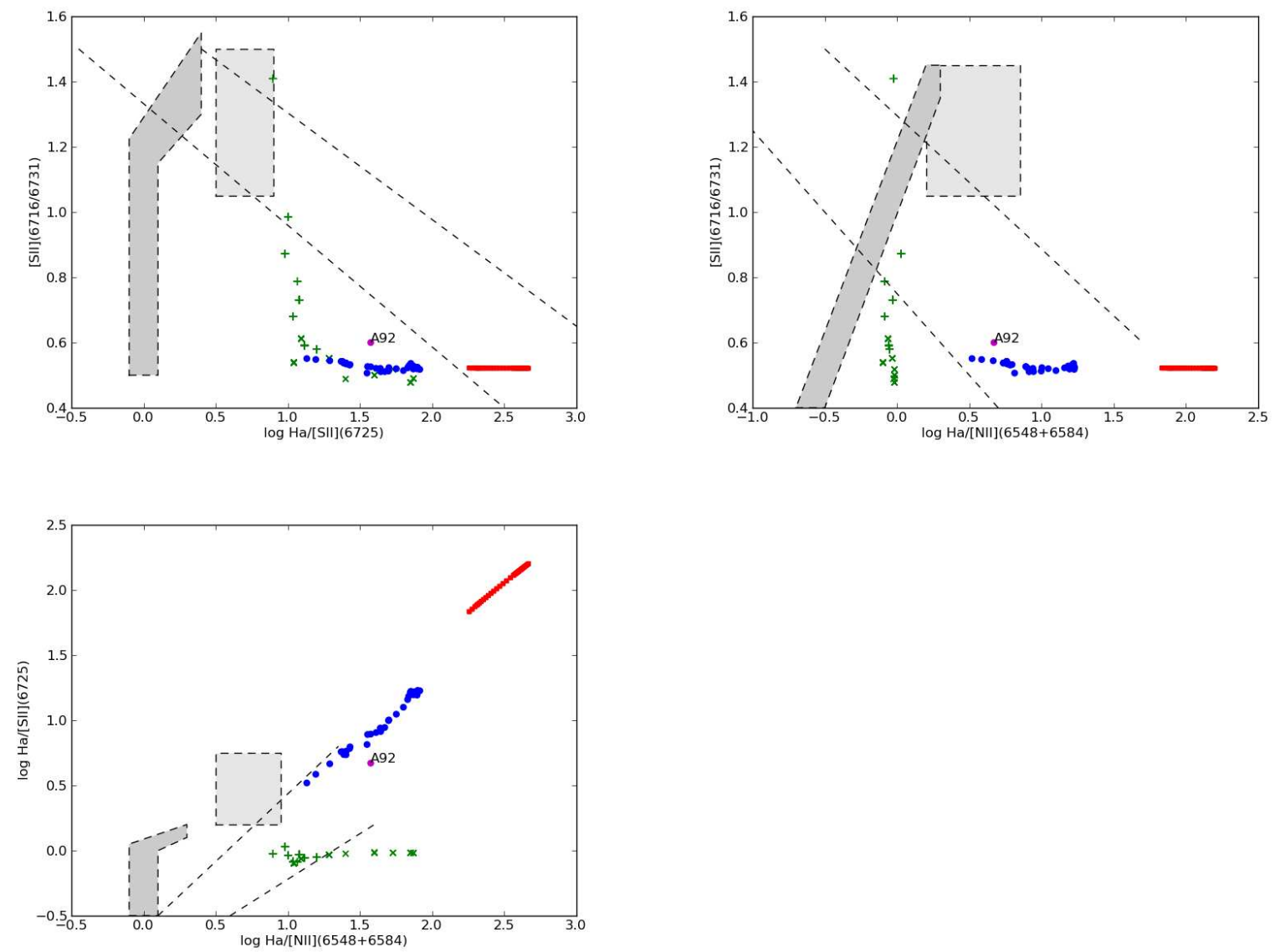

Figura 4.22: Diagrama de diagnóstico de plasma para IC4776, representando comportamento diverso dos modelos gerados. Em escuro, a região típica de remanescentes de supernova (RSN), em cinza claro a região de regiões HII, e entre as retas as regiões de NPs. Os pontos observacionais das NPs são exibidos como "+" (regiões externas) e "x" (regiões internas), comparados com os modelos.

Um caso claro é o de IC4776 no diagrama $[S I I] \lambda 6716 / \lambda 6731 \times \log H \alpha /[N I I](\lambda 6548+$ 6584) (figura 4.22). Enquanto que os modelos prevêem um comportamento próximo ao horizontal, as observações das regiões centrais aproximam-se do esperado pelos modelos construídos. Neste objeto os valores observacionais estão fora da região esperada para NPs, mas por um pequeno fator estão em concordância com o valor dos fluxos de catálogo. 
Capítulo 5

\section{Conclusões}

Através das técnicas observacionais empregadas pode-se resolver angularmente os fluxos emitidos na banda óptica do espectro, e consequentemente investigar variações das propriedades físicas para esta classe de objeto astronômico. Destaca-se que os fluxos emergentes de uma NP não são homogêneos, mas variam ao longo de sua estrutura angular (e consequentemente, de sua estrutura espacial).

Tais dados fornecem um valioso recurso para o estudo das propriedades intrínsecas dos objetos, utilizados em conjunto com modelos numéricos de fotoionização e vínculos complementares a partir de outras técnicas de observação.

Em tais simulações é possível reproduzir em boa medida o quadro de observações realizadas, mesmo a partir de considerações simplificadoras para a construção de ambiente astrofísico como campo de radiação predominantemente radial, geometria simétrica e homogênea, campo de velocidades estático, entre outras.

As duas técnicas mostraram-se de certa forma complementares na caracterização de um conjunto de NPs: os objetos mais extensos selecionados para a técnica de fenda longa são os que possuem maiores dimensões espaciais, e consequentemente menor densidade eletrônica; enquanto que os objetos de campo integral, selecionados pelo alto brilho superficial, são os que possuem maiores densidades de matéria, porém com menores dimensões espaciais.

Sem a pretensão de apresentar o quadro completo do espectro das NPs individualmente, mas sim a reprodução das propriedades de suas regiões mais brilhantes na parte óptica, estabelece-se limites para os parâmetros do objeto, tais como características da distribuição de matéria, propriedades da fonte central, distância e dimensões envolvidas.

Dos seis objetos com simulações concluídas neste trabalho, quatro indicam ser NPs 
limitadas por densidade a partir de dois importantes vínculos: razão de fluxos de linhas [NII] $/ \mathrm{H} \alpha$ e fluxo total $\mathrm{H} \beta$, dentro dos limites de densidades eletrônicas observados pelas linhas de [SII]. Isto porque dentre as hipóteses adotadas, a limitação por densidade é o principal mecanismo para se reduzir o valor do fluxo total.

Se isto for confirmado, a proporção encontrada para esta classe de NPs comparada com as NPs limitadas por ionização, mesmo estatisticamente limitada, contraria a premissa de que a maioria das NPs são limitadas por radiação. Isto porque numa amostra onde os objetos são selecionados por seu brilho no óptico, espera-se encontrar em maior número de NPs limitadas por ionização. Para um destes objetos, NGC5844, não foi possível reproduzir o baixo fluxo total $\mathrm{H} \beta$ observado. Mesmo assim, a possibilidade deste ser limitado por radiação é praticamente descartada, uma vez que tais modelos apresentam um fluxo total esperado uma ordem de grandeza maior que o observado.

Como aponta Kwok (2000), a premissa de que as NPs são limitadas por ionização é chave para a determinação das luminosidades e temperaturas das ECNPs a partir de propriedades nebulares. A ampla variação da densidade entre os pólos e o equador indica que uma NP pode ser limitada por ionização na direção equatorial mas não na direção polar. A detecção de débeis halos através de imageamento de banda estreita (Corradi et al., 2003) sugere que muitas NPs estão totalmente ionizadas (i.e., são limitadas por densidade). Em análises estatísticas de NPs é de grande importância o conhecimento da ocorrência de NPs limitadas por densidade em relação à de NPs limitadas por ionização.

O estudo das variações internas de emissão nas NPs em diagramas de diagnóstico de plasma utilizados por Sabbadin et al. (1977) mostrou-se produtiva. Os fatores que levam à discrepância na distribuição dos pontos observacionais das regiões classicamente esperadas devem estar associados com a não convergência das simulações calculadas para este trabalho. Isso é ainda mais relevante uma vez que nos objetos para os quais se obteve bons modelos, o comportamento dos pontos observacionais é o mais próximo do que se obtém pelas simulações, e que resultaram em bons modelos limitados por ionização. Em especial, as assimetrias presentes na nuvem (eventualmente realçando a influência da componente não radial da radiação, como sobredensidades localizadas), configurações geométricas mais complexas, ou mesmo a ocorrência de choques fornecendo energia ao gás (e consequentemente alterando sua emissividade) podem estar associados à origem deste 
comportamento distinto.

Como se conseguiu a convergência entre modelo e observação em ambas as instrumentações, pode-se afirmar que a convergência ou não dos modelos propostos e comportamento nos diagramas de diagnóstico não se trata de eventual tendência introduzida pela instrumentação utilizada, mas fatores intrínsecos aos objetos e que não são contempladas com as hipóteses assumidas.

Dentro da resolução da instrumentação, subestruturas puderam ser identificadas em objetos analisados como desvios na simetria dos fluxos emergentes. Sob espectroscopia de campo integral, destacam-se em NGC5882 e IC2553, associados a sub-estruturas presentes no imageamento de alta resolução e em echelogramas, respectivamente. Em IC1297, dada a ausência deste tipo de informação para o objeto, apenas sugere-se a possibilidade como fator de assimetria.

O método de FCIs, amplamente empregado para análise da composição química média a partir dos fluxos totais emergentes possui restrições quando empregado na determinação de abundâncias de regiões espaciais específicas do plasma. Em particular, neste trabalho encontramos uma sistemática determinação de sobreabundâncias nas regiões externas do plasma, onde se espera baixa intensidade de radiação e onde se encontrariam as chamadas frentes de ionização. Assim, para objetos extensos, os valores de composição química médios podem variar dependendo do posicionamento da fenda sobre o objeto. Entretanto, há uma boa concordância dos parâmetros médios com os valores das regiões centrais, mais brilhantes.

A variação de $\mathrm{E}(\mathrm{B}-\mathrm{V})$ observada ao longo da estrutura angular dos objetos sob técnica de espectroscopia de fenda longa é intrigante. A contaminação do contínuo de estrelas, que apresentam fortes bandas de absorção nas linhas do Hidrogênio, pode estar na origem desta variação. Muitas regiões apresentam valor de excesso de cor determinado pela razão de fluxos $\mathrm{H} \alpha / \mathrm{H} \beta$ muito diferente do valor obtido a partir de fluxos médios, e com diferenças maiores que os erros esperados nas determinações dos fluxos angulares. Tais variações são maiores também do que se pode prever pela teoria de recombinação do Hidrogênio $(|\Delta \mathrm{E}(\mathrm{B}-\mathrm{V})| \leq 0,1$ - diferenças entre ambientes de densidades e temperaturas extremos) e indicam que a extinção diferencial realmente está ocorrendo na nuvem, uma vez que apresentam valores compatíveis de fluxos e parâmetros físicos calculados a partir de modelos 
de fotoionização. O valor de $\Delta \mathrm{E}(\mathrm{B}-\mathrm{V})$ acima é determinado a partir da comparação com a maior razão $\mathrm{H} \alpha / \mathrm{H} \beta$ esperada (3,04 para um gás de baixa densidade e baixa temperatura - $10^{2} \mathrm{e}^{-} / \mathrm{cm}^{3}$ em $\left.5.000 \mathrm{~K}\right)$ em oposição à menor razão (2,73 para gás de alta densidade $\mathrm{e}$ temperatura - $10^{6} \mathrm{e}^{-} / \mathrm{cm}^{3}$ em 20.000K). Os valores são de Osterbrock (1989).

A origem de tal extinção pode ser relacionada com a presença não homogênea de grãos e poeira nas NPs. Uma forma de se avaliar tal influência seria a partir de simulações de outras faixas espectrais além do óptico, tais como ultravioleta (UV), infravermelho (IV), rádio e observações polarimétricas.

\subsection{Perspectivas}

A inclusão de mais vínculos nas simulações, como observações realizadas em outras bandas do espectro, pode gerar melhores e mais completos modelos dos objetos. Em particular, a inclusão de fluxos no IV pode ser particularmente interessante para o estudo da influência de poeira na radiação emergente da nuvem, como discutido na seção anterior.

Echelogramas e perfis de linhas também são interessantes ferramentas para a determinação da distribuição de matéria na NP, permitindo avaliar também o campo de velocidades do objeto (e eventualmente a evolução da distribuição de matéria). Os recursos de análise de perfil de linhas e echelogramas estão disponíveis na ferramenta Cloudy_3D, porém não foram utilizados nesta parte do projeto. Assim, os cenários de distribuição de matéria e variação de densidades na estrutura espacial do objeto não estão esgotados, mas podem ser explorados com a inclusão de vínculos adequados. A distribuição de matéria na nuvem é dos parâmetros de maior influência na variação angular do fluxo emergente.

A inclusão de mais objetos pode contribuir para uma análise de propriedades que sejam estatisticamente relevantes para as NPs. Além da questão das condições do limite da nuvem, que se evidenciou nas simulações, um conjunto de objetos pode gerar por exemplo uma classificação morfológica mais precisa, baseada não somente no formato projetado na nuvem.

As variações determinadas observacionalmente nos parâmetros físicos, comparadas aqui com modelos homogêneos, podem ainda ser comparadas com a previsão de modelos nãohomogêneos, tais como modelos hidrodinâmicos que levem em conta a ocorrência de turbulências nos objetos. A inclusão de tais variações são indicadas para códigos de fo- 
toionização realmente tridimensionais, que podem tratar de uma maneira consistente o problema das regiões de "sombra", regiões onde a radiação incidente é consideravelmente de origem não radial. 


\section{Referências Bibliográficas}

Abell G. O., Goldreich P., On the Origin of Planetary Nebulae, PASP, 1966, vol. 78, p. 232

Acker A., Marcout J., Ochsenbein F., Stenholm B., Tylenda R., Strasbourg - ESO catalogue of galactic planetary nebulae. Part 1; Part 2, 1992

Alexander J., Balick B., Nebular Abundance Errors., AJ, 1997, vol. 114, p. 713

Balick B., The evolution of planetary nebulae. I - Structures, ionizations, and morphological sequences, AJ, 1987, vol. 94, p. 671

Cardelli J. A., Clayton G. C., Mathis J. S., The relationship between infrared, optical, and ultraviolet extinction, ApJ, 1989, vol. 345, p. 245

Cazetta J. O., Nebulosas Planetárias e suas estrelas centrais: evolução e distâncias, Universidade de São Paulo, 1999, Tese de Doutorado, 131 p.

Corradi R. L. M., Gonçalves D. R., Villaver E., Mampaso A., Perinotto M., Knots in the Outer Shells of the Planetary Nebulae IC 2553 and NGC 5882, ApJ, 2000, vol. 542, p. 861

Corradi R. L. M., Schönberner D., Steffen M., Perinotto M., Ionized haloes in planetary nebulae: new discoveries, literature compilation and basic statistical properties, MNRAS, 2003, vol. 340 , p. 417

Dayal A., Sahai R., Watson A. M., Trauger J. T., Burrows C. J., Stapelfeldt K. R., Gallagher III J. S., The Etched Hourglass Nebula MYCN 18. II. A Spatio-kinematic Model, AJ, 2000, vol. 119, p. 315 
de Freitas-Pacheco J. A., Barbuy B., Costa R. D. D., Idiart T. E. P., Type-I Planetary

Nebulae in the Large Magellanic Cloud - Oxygen Sulphur and Argon Abundances as Tracers of Chemical Enrichment, A\&A, 1993, vol. 271, p. 429

de Freitas Pacheco J. A., Maciel W. J., Costa R. D. D., Chemical abundances of disk planetary nebulae, A\&A, 1992, vol. 261, p. 579

Ercolano B., Young P. R., Drake J. J., Raymond J. C., X-Ray Enabled MOCASSIN: A Three-dimensional Code for Photoionized Media, ApJS, 2008, vol. 175, p. 534

Ferland G. J., Quantitative Spectroscopy of Photoionized Clouds, ARA\&A, 2003, vol. 41, p. 517

Ferland G. J., Korista K. T., Verner D. A., Ferguson J. W., Kingdon J. B., Verner E. M., CLOUDY 90: Numerical Simulation of Plasmas and Their Spectra, PASP, 1998, vol. 110, p. 761

Fitzpatrick E. L., Correcting for the Effects of Interstellar Extinction, PASP, 1999, vol. 111, p. 63

Gathier R., Pottasch S. R., Properties of planetary nebulae. II - Central star evolution, A\&A, 1989, vol. 209, p. 369

Girard P., Köppen J., Acker A., Chemical compositions and plasma parameters of planetary nebulae with Wolf-Rayet and wels type central stars, A\&A, 2007, vol. 463, p. 265

Gonçalves D. R., Corradi R. L. M., Mampaso A., Low-Ionization Structures in Planetary Nebulae: Confronting Models with Observations, ApJ, 2001, vol. 547, p. 302

Gonçalves D. R., Corradi R. L. M., Mampaso A., Perinotto M., The Physical Parameters, Excitation, and Chemistry of the Rim, Jets, and Knots of the Planetary Nebula NGC 7009, ApJ, 2003, vol. 597, p. 975

Gonçalves D. R., Mampaso A., Corradi R. L. M., Perinotto M., Riera A., López-Martín L., K 4-47: a planetary nebula excited by photons and shocks, MNRAS, 2004, vol. 355, p. 37 
Górny S. K., Schwarz H. E., Corradi R. L. M., Van Winckel H., An atlas of images of Planetary Nebulae, A\&AS, 1999, vol. 136, p. 145

Gray R. O., Corbally J. C., Stellar Spectral Classification, 2009

Gruenwald R., Viegas S. M., Broguiere D., A New Generation of Photoionization Codes: Three-dimensional Models. The Bipolar Planetary Nebula IC 4406, ApJ, 1997, vol. 480, p. 283

Gurzadyan G. A., The Physics and Dynamics of Planetary Nebulae, 1997

Hajian A. R., Movit S. M., Trofimov D., Balick B., Terzian Y., Knuth K. H., GranquistFraser D., Huyser K. A., Jalobeanu A., McIntosh D., Jaskot A. E., Palen S., Panagia N., An Atlas of [N II] and [O III] Images and Spectra of Planetary Nebulae, ApJS, 2007, vol. 169 , p. 289

Helene O. A. M., Vanin V. R., Tratamento estatístico de dados em física experimental, 1981,1981

Herald J. E., Bianchi L., Far-Ultraviolet Spectroscopic Analyses of Four Central Stars of Planetary Nebulae, ApJ, 2004, vol. 609, p. 378

Hyung S., Aller L. H., Feibelman W. A., The Spectrum of the Planetary Nebula NGC6572, MNRAS, 1994, vol. 269, p. 975

Kahn F. D., West K. A., Shapes of planetary nebulae, MNRAS, 1985, vol. 212, p. 837

Kaler J. B., The evolution of large planetary nebulae and their central stars, ApJ, 1983, vol. 271 , p. 188

Kaler J. B., Jacoby G. H., Central star temperatures of optically thick planetary nebulae and a distance-independent test of dredge-up theory, ApJ, 1989, vol. 345, p. 871

Khromov G. S., Planetary nebulae, Space Sci. Rev., 1989, vol. 51, p. 339

Kingdon J., Ferland G. J., Collisional effects in He I: an observational analysis, ApJ, 1995, vol. 442 , p. 714 
Kingsburgh R. L., Barlow M. J., Elemental abundances for a sample of southern galctic planetary nebulae., MNRAS, 1994, vol. 271, p. 257

Kwok S., The Origin and Evolution of Planetary Nebulae, 2000

Kwok S., Purton C. R., Fitzgerald P. M., On the origin of planetary nebulae, ApJ, 1978, vol. 219, p. L125

Mal'Kov Y. F., A self-consistent determination of the distances, physical parameters, and chemical composition for a large sample of galactic planetary nebulae: The distances and parameters of central stars and the optical depths of envelopes, Astronomy Reports, 1997, vol. 41, p. 760

McKenna F. C., Keenan F. P., Kaler J. B., Wickstead A. W., Bell K. L., Aggarwal K. M., [N II] and [O III] Mean Electron Temperatures in Planetary Nebulae, PASP, 1996, vol. 108 , p. 610

Monteiro H. S. A., Nebulosas Planetárias anulares: o caso NGC 3132, Universidade de São Paulo, 2000, Dissertação de Mestrado, 101 p.

Monteiro H. S. A., Estrutura Espacial de Nebulosas Planetárias, Universidade de São Paulo, 2004, Tese de Doutorado, 181 p.

Morisset C., Cloudy_3D, a new pseudo-3D photoionization code. In Planetary Nebulae in our Galaxy and Beyond, vol. 234 of IAU Symposium, 2006, p. 467

Osterbrock D. E., Astrophysics of Gaseous Nebulae and Active Galactic Nuclei. University Science Books, 1989, 408 p.

Peimbert M., Chemical abundances in planetary nebulae. In Planetary Nebulae, vol. 76 of IAU Symposium, 1978, p. 215

Pequignot D., Petitjean P., Boisson C., Total and effective radiative recombination coefficients, A\&A, 1991, vol. 251, p. 680

Perinotto M., Chemical abundances in planetary nebulae - Basic data and correlations between elements, ApJS, 1991, vol. 76, p. 687 
Phillips J. P., Electron densities in planetary nebulae, and the unusual characteristics of the [S BT II] emission zone and densities in planetary nebulae, A\&A, 1998, vol. 340, p. 527

Phillips J. P., Cuesta L., Density and Excitation Mapping of M2-9, AJ, 1999, vol. 118, p. 2919

Pottasch S. R., Bernard-Salas J., Planetary nebulae abundances and stellar evolution, A\&A, 2006, vol. 457, p. 189

Preite-Martinez A., Acker A., Koeppen J., Stenholm B., The Energy-Balance temperature of central stars of galactic planetary nebulae, A\&AS, 1989, vol. 81, p. 309

Preite-Martinez A., Acker A., Koeppen J., Stenholm B., The energy-balance temperature of central stars of galactic planetary nebulae. II, A\&AS, 1991, vol. 88, p. 121

Rauch T., A grid of synthetic ionizing spectra for very hot compact stars from NLTE model atmospheres, A\&A, 2003, vol. 403, p. 709

Sabbadin F., Minello S., Bianchini A., Sharpless 176: a large, nearby planetary nebula., A\&A, 1977, vol. 60, p. 147

Sabbadin F., Turatto M., Ragazzoni R., Cappellaro E., Benetti S., The structure of planetary nebulae: theory vs. practice, A\&A, 2006, vol. 451, p. 937

Schonberner D., Structure and evolution of central stars of Planetary Nebulae (Invited Review). In Planetary Nebulae, vol. 180 of IAU Symposium, 1997, p. 379

Schwarz H. E., Corradi R. L. M., Melnick J., A catalogue of narrow band images of planetary nebulae, A\&AS, 1992, vol. 96, p. 23

Torres-Peimbert S., Peimbert M., Physical conditions in two halo planetary nebulae, Rev. Mexicana Astron. Astrofis., 1979, vol. 4, p. 341

Tsamis Y. G., Barlow M. J., Liu X., Danziger I. J., Storey P. J., A deep survey of heavy element lines in planetary nebulae - I. Observations and forbidden-line densities, temperatures and abundances, MNRAS, 2003, vol. 345, p. 186 
Tylenda R., Acker A., Stenholm B., Koeppen J., The extinction constants for galactic planetary nebulae, A\&AS, 1992, vol. 95, p. 337

Webster B. L., Emission-line fluxes of planetary nebulae and related objects, PASP, 1983, vol. 95 , p. 610

Zepeda M. E., Modelos 3D de Fotoionización de la Nebulosa Planetaria MyCn18, Hourglass, Universidad Nacional Autonoma de Mexico, 2006, Tese de Doutorado, 78 p.

Zhang C. Y., Kwok S., Trace of planetary nebula evolution by distance-independent parameters, ApJS, 1993, vol. 88, p. 137 
Apêndice 

Apêndice A

\section{O script de redução PNPACK}

Com o intuito de facilitar a análise do grande volume de dados, bem como na tentativa de estabelecer um critério sistemático para a obtenção dos fluxos das linhas, alternativamente ao procedimento manual, o pacote PNPACK foi planejado e escrito, em conjunto com o aluno Oscar Cavichia.

A análise dos dados para este projeto (espectroscopia de fenda longa) foi realizada no ambiente IRAF. A redução é feita com o pacote $N O A O$ e seus subpacotes, enquanto que o cálculo de abundâncias iônicas e parâmetros físicos é feito com o subpacote NEBULAR do STSDAS. Ao longo do projeto também analisamos dados de Nebulosas Planetárias sob UCI, sendo um script como este fundamental para adequada análise dos dados.

Abaixo são descritas as rotinas realizadas pelo pacote, escritas para os dados do telescópio do OPD. A atual versão também suporta os dados do telescópio SOAR.

pnreduc Redução completa dos dados, desde correção de bias à calibração em fluxo, incluindo redução de estrelas-padrão. Há opção para controle de todas as etapas da redução.

pnoncered Redução dos dados como anterior, mas para estrelas-padrão já reduzidas.

pnderedden Correção de avermelhamento, com as funções Cardelli et al. (1989) e Fitzpatrick (1999) com a razão $H_{\alpha} / H_{\beta}=2.85$. Obtém o valor de $\mathrm{E}(\mathrm{B}-\mathrm{V})$ pelas linhas do $\mathrm{H}$ para uma das curvas, ou força um valor como parâmetro ou como arquivo de entrada. Também adiciona a informação de deslocamento médio em velocidade radial do es-

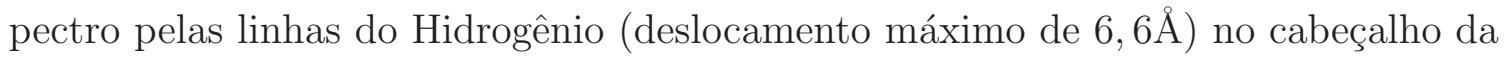
imagem, com o parâmetro "PNSHIFT". Coloca informação do avermelhamento no 
cabeçalho em "PNDERED" se Fitzpatrick (1999), ou padrão NOAO/DEREDDEN se Cardelli et al. (1989).

pnflux Calcula os fluxos das linhas mais comuns em Nebulosas Planetárias (ver tabela A.1). Pode-se levar ou não em conta o deslocamento do espectro calculado em pnderedden.

pncalcs Calcula os fluxos em escala $H_{\beta}=100$, as abundâncias iônicas para cada linha, e as abundâncias iônicas médias e elementares, além dos parâmetros físicos. Pode-se forçar o valor dos parâmetros físicos por um arquivo de entrada (o mesmo pode ser feito para os fluxos, editando o arquivo de fluxos da rotina pnflux). Calcula também a média dos parâmetros físicos e abundâncias se houver duas ou mais exposições, e reúne as médias das várias aberturas em um único arquivo.

O passo mais sensível da análise dos dados é justamente a obtenção de um valor de fluxo confiável para a linha. Por isso, são feitas 5 tentativas para o ajuste gaussiano da linha, cada uma das vezes em níveis distintos de contínuo. Para os fluxos serem aceitos como válidos, em pnderedden, o valor absoluto de $\left(\lambda_{\text {ajuste }}-\lambda_{\text {teorico }}\right)$ deve ser $<6,7 \AA$ Aara $H \alpha$ e $<$ 4, $9 \AA$ Aिpara $H \beta$. Ambos valores limites equivalem a velocidade radial de $300 \mathrm{~km} / \mathrm{s}$ (supondo deslocamento instrumental nulo). Em pnflux, o valor absoluto de $\left(\lambda_{\text {ajuste }}-\lambda_{\text {teorico }}\right)$ deve ser $<4,0 \AA$ para qualquer linha. Em ambos os casos, a linha só é aceita de fluxo $F>0$ e o valor de largura à meia-altura $F W H M<25 \AA$. Se os 5 ajustes foram feitos com sucesso, computa-se o valor de fluxo mediano. Se menos ajustes são aceitos, o fluxo computado é a média dos ajustes. É considerada como ruído a linha que em um único ajuste, o fluxo obtido for maior ou menor que a média dos ajustes anteriores por um fator 2 ou mais (neste caso, o fluxo também não é computado). 
Tabela A.1 - Linhas cujos fluxos são procurados por padrão pela rotina pnflux em espectros no visível.

\begin{tabular}{|c|c|c|c|}
\hline linha $(\AA)$ & espécie atom. & linha $(\AA)$ & espécie atom. \\
\hline 3727. & $\mathrm{OII}+\mathrm{SIII}$ & 5412. & HeII \\
\hline 3798. & HI & 5518. & $\mathrm{Cl} 3$ \\
\hline 3820. & $\mathrm{HeI}$ & 5538. & $\mathrm{Cl} 3$ \\
\hline 3835. & HI & 5755. & NII \\
\hline 3869. & $\mathrm{NeIII}+\mathrm{HeI}$ & 5876. & $\mathrm{HeI}$ \\
\hline 3889. & $\mathrm{HI}+\mathrm{HeI}$ & 6300. & OI \\
\hline 3968. & $\mathrm{NeIII+HI}$ & 6312. & SIII \\
\hline 4026. & $\mathrm{HeI}$ & 6435. & $\mathrm{ArV}$ \\
\hline 4074 & SII & 6548. & NII \\
\hline 4102. & $\mathrm{H} \delta$ & 6563. & $\mathrm{H} \alpha$ \\
\hline 4267. & CII & 6584. & NII \\
\hline 4300 & FeII & 6678. & $\mathrm{HeI}$ \\
\hline 4341. & $\mathrm{H} \gamma$ & 6716. & SII \\
\hline 4363. & OIII & 6731. & SII \\
\hline 4471. & $\mathrm{HeI}$ & 7005 & $\mathrm{ArV}$ \\
\hline 4686. & HeII & 7065. & $\mathrm{HeI}$ \\
\hline 4711. & $\mathrm{ArIV}+\mathrm{HeI}$ & 7135. & ArIII \\
\hline 4740. & ArIV & 7237. & ArIV \\
\hline 4861. & $\mathrm{H} \beta+\mathrm{HeII}$ & 7263. & ArIV \\
\hline 4922. & $\mathrm{HeI}$ & 7281. & $\mathrm{HeI}$ \\
\hline 4959. & OIII & 7325. & OII $x 2$ \\
\hline 5007. & OIII & 7532. & $\mathrm{Cl} 4$ \\
\hline 5192. & ArIII & 7751. & ArIII \\
\hline 5199. & NI & & \\
\hline
\end{tabular}




\section{Apêndice B}

\section{Dados observacionais do projeto}

Tabela B.1 - Lista dos objetos observados em missões ao OPD/LNA.

\begin{tabular}{|c|c|c|c|c|}
\hline Objeto & Noite & Fendas & Aberturas & Ang. $\mathrm{N} \rightarrow \mathrm{L}$ \\
\hline NGC246 & $24 / 08 / 08$ & 1 & 18 & $90^{\circ}$ \\
\hline NGC1535 & $22 / 08 / 08$ & 1 & 8 & $90^{\circ}$ \\
\hline NGC 2899 & $17 / 05 / 09$ & 1 & 16 & $119,5^{\circ}$ \\
\hline \multirow{2}{*}{ NGC3132 } & $16 / 05 / 07$ & 5 & 8 & $63,5^{\circ}$ \\
\hline & $14 / 05 / 09$ & 3 & $"$ & $"$ \\
\hline IC4406 & $17 / 05 / 07$ & 1 & 14 & $90^{\circ}$ \\
\hline NGC 3242 & $16 / 06 / 09$ & 3 & 9 & $145,5^{\circ}$ \\
\hline NGC 3699 & $13 / 05 / 09$ & 1 & 8 & $130,5^{\circ}$ \\
\hline NGC 4071 & $13 / 05 / 09$ & 1 & 9 & $120,5^{\circ}$ \\
\hline NGC 4361 & $16 / 06 / 09$ & 1 & 16 & $36,5^{\circ}$ \\
\hline \multirow{2}{*}{ NGC5189 } & $15 / 05 / 07$ & 3 & 16 & $59^{\circ}$ \\
\hline & $16 / 05 / 09$ & $"$ & $"$ & "० \\
\hline NGC5844 & $15 / 05 / 07$ & 3 & 11 & $90^{\circ}$ \\
\hline NGC5873 & $17 / 05 / 09$ & 1 & 5 & $90^{\circ}$ \\
\hline \multirow{2}{*}{ NGC5882 } & $16 / 05 / 07$ & 3 & 7 & $90^{\circ}$ \\
\hline & $17 / 05 / 09$ & 3 & 7 & $21,5^{\circ}$ \\
\hline \multirow{2}{*}{ NGC5979 } & $17 / 05 / 07$ & 1 & 5 & $110^{\circ}$ \\
\hline & $13 / 05 / 09$ & $"$ & $"$ & "० \\
\hline IC4599 & $17 / 05 / 07$ & 1 & 4 & $90^{\circ}$ \\
\hline NGC6302 & $17 / 05 / 07$ & 1 & 14 & $90^{\circ}$ \\
\hline NGC6309 & $17 / 05 / 07$ & 1 & 7 & $90^{\circ}$ \\
\hline NGC 6369 & $16 / 05 / 09$ & 1 & 10 & $69^{\circ}$ \\
\hline IC4663 & $16 / 05 / 07$ & 1 & 5 & $71,5^{\circ}$ \\
\hline \multirow{2}{*}{ NGC6445 } & $16 / 05 / 07$ & 1 & 10 & $47,5^{\circ}$ \\
\hline & $13 / 05 / 09$ & 3 & 8 & $43^{\circ}$ \\
\hline NGC 6629 & $16 / 05 / 09$ & 1 & 5 & $137,5^{\circ}$ \\
\hline NGC7009 & $17 / 05 / 07$ & 1 & 9 & $79^{\circ}$ \\
\hline IC5148 & $22 / 08 / 08$ & 3 & 18 & $90 / 30 /-30^{\circ}$ \\
\hline
\end{tabular}

Continua na próxima pagina... 
Tabela B.1 - Continuação

\begin{tabular}{ccccc}
\hline Objeto & Noite & Fendas & Aberturas & Ang.N $\rightarrow \mathrm{L}$ \\
\hline MZ1 & $17 / 05 / 09$ & 1 & 12 & $156,5^{\circ}$ \\
\hline \multirow{2}{*}{ MZ2 } & $15 / 05 / 07$ & 1 & 7 & $90^{\circ}$ \\
& $13 / 05 / 09$ & 2 & 7 & $85,5 /-4,5^{\circ}$ \\
\hline M2-9 & $16 / 05 / 07$ & 1 & 7 & $0^{\circ}$ \\
\hline Hb 5 & $16 / 05 / 09$ & 1 & 10 & $78,5^{\circ}$ \\
& & & & \\
\hline
\end{tabular}

As observações com a instrumentação de campo integral só ocorreram na parte final do projeto, em virtude da indisponibilidade do uso da instrumentação.

Tabela B.2 - Observações do projeto com a técnica de espectroscopia de campo integral.

\begin{tabular}{|c|c|c|c|c|}
\hline PNG & Nome & Data & $\lambda$ central & Integracoes \\
\hline \multirow{3}{*}{ 002.0-13.4 } & \multirow{3}{*}{ IC4776 } & $08 / 06 / 10$ & 6550 & $5 \times 900 \mathrm{~s}$ \\
\hline & & $12 / 06 / 10$ & 5650 & $5 \times 600 \mathrm{~s}$ \\
\hline & & $13 / 06 / 10$ & 4650 & $3 \times 1200$ s \\
\hline \multirow{3}{*}{$002.7-04.8$} & \multirow{3}{*}{ M1-42 } & $07 / 06 / 10$ & 6550 & $2 \times 900 \mathrm{~s}$ \\
\hline & & $09 / 06 / 10$ & 6550 & $3 \times 900 s$ \\
\hline & & $12 / 06 / 10$ & 5650 & $2 \mathrm{x} 1200 \mathrm{~s}$ \\
\hline \multirow{3}{*}{ 025.8-17.9 } & \multirow{3}{*}{ NGC6818 } & $07 / 06 / 10$ & 6550 & $2 \times 600 \mathrm{~s}$ \\
\hline & & $12 / 06 / 10$ & 5650 & $2 \mathrm{x} 1200 \mathrm{~s}$ \\
\hline & & $13 / 06 / 10$ & 4650 & $2 \times 1200 s$ \\
\hline \multirow{3}{*}{$034.5-06.7$} & \multirow{3}{*}{ NGC6778 } & $07 / 06 / 10$ & 6550 & $2 \mathrm{x} 1200 \mathrm{~s}$ \\
\hline & & $12 / 06 / 10$ & 5650 & $2 \mathrm{x} 1200 \mathrm{~s}$ \\
\hline & & $13 / 06 / 10$ & 4650 & $2 \times 1200 \mathrm{~s}$ \\
\hline \multirow{3}{*}{$034.6+11.8$} & \multirow{3}{*}{ NGC6572 } & $08 / 06 / 10$ & 6550 & $5 \times 450 \mathrm{~s}$ \\
\hline & & $12 / 06 / 10$ & 5650 & $5 \times 300 \mathrm{~s}$ \\
\hline & & $13 / 06 / 10$ & 4650 & $3 x 1200 s$ \\
\hline \multirow{2}{*}{ 285.4-05.3 } & \multirow{2}{*}{ IC 2553} & $08 / 06 / 10$ & 6550 & $5 \times 1200 \mathrm{~s}$ \\
\hline & & $12 / 06 / 10$ & 5650 & $3 \times 1200 \mathrm{~s}$ \\
\hline \multirow{3}{*}{$304.5-04.8$} & \multirow{3}{*}{ IC4191 } & $09 / 06 / 10$ & 6550 & $3 \times 1200 s$ \\
\hline & & $11 / 06 / 10$ & 5650 & $2 \mathrm{x} 1200 \mathrm{~s}$ \\
\hline & & $13 / 06 / 10$ & 4650 & $3 \times 1200 s$ \\
\hline \multirow{3}{*}{$327.8+10.0$} & \multirow{3}{*}{ NGC5882 } & $08 / 06 / 10$ & 6550 & $5 \times 1200 \mathrm{~s}$ \\
\hline & & $12 / 06 / 10$ & 5650 & $3 \mathrm{x} 1200 \mathrm{~s}$ \\
\hline & & $13 / 06 / 10$ & 4650 & $2 \times 1200 s$ \\
\hline \multirow{2}{*}{$358.3-21.6$} & & $08 / 06 / 10$ & 6550 & $3 \times 900 \mathrm{~s}$ \\
\hline & IC1297 & $13 / 06 / 10$ & 4650 & $4 \mathrm{x} 1200 \mathrm{~s}$ \\
\hline
\end{tabular}




\section{Apêndice $\mathrm{C}$}

\section{Tabela de fluxos obtidos com espectroscopia de fenda-longa}

Tabela C.1 - Fluxos observados por toda fenda e respectivas incertezas.

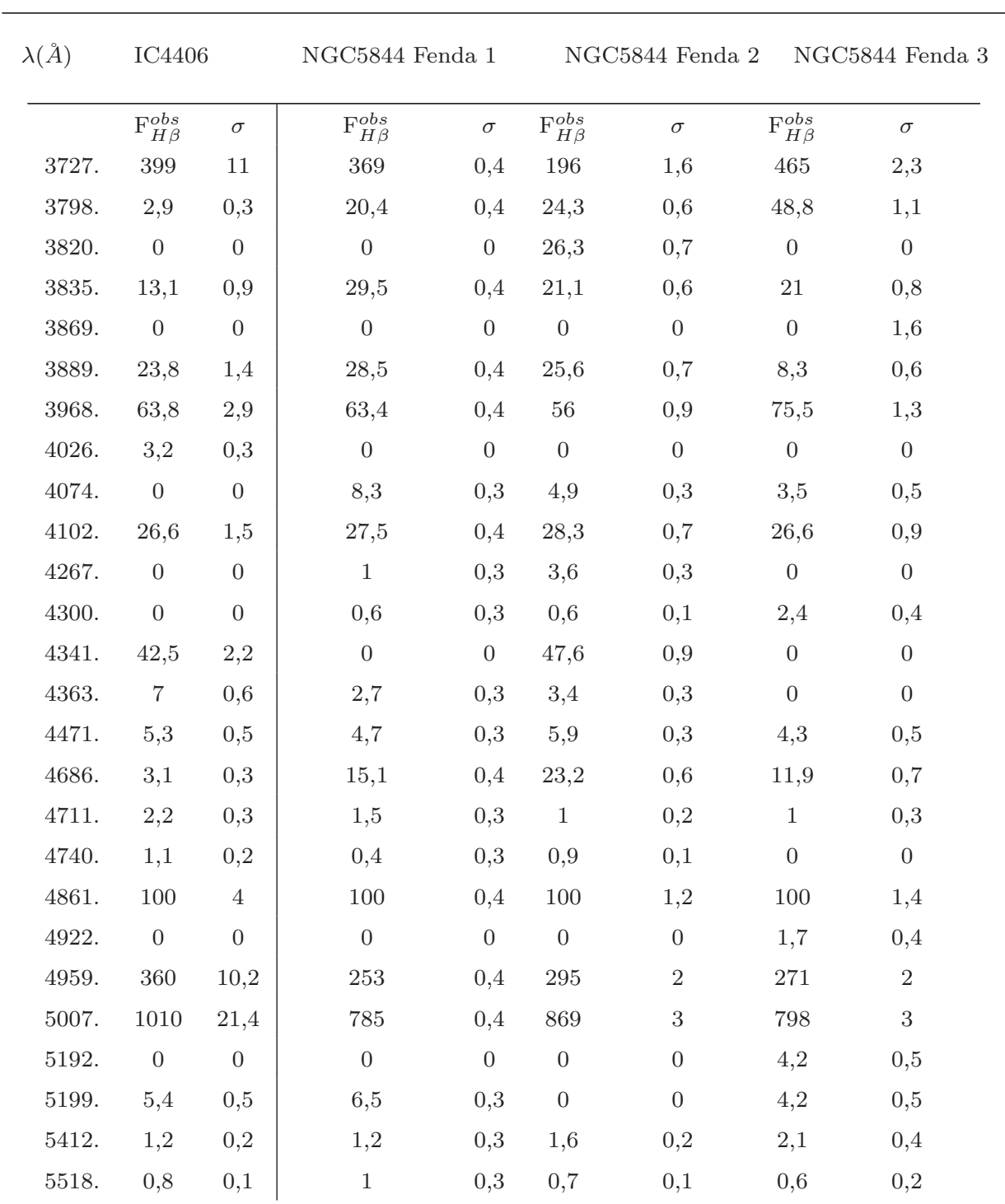

Continua na próxima página... 
Tabela C.1 - Continuação

\begin{tabular}{|c|c|c|c|c|c|c|c|c|}
\hline \multirow{2}{*}{$\begin{array}{l}\lambda(\AA) \\
\quad 5538 .\end{array}$} & \multicolumn{2}{|c|}{ IC4406 } & \multicolumn{2}{|c|}{ NGC5844 Fenda 1} & \multicolumn{2}{|c|}{ NGC5844 Fenda 2} & \multicolumn{2}{|c|}{ NGC5844 Fenda 3} \\
\hline & 0,6 & 0,1 & 0,7 & 0,3 & 0,5 & 0,1 & 0,3 & 0,2 \\
\hline 5755. & 6 & 0,5 & 3,7 & 0,3 & 4,2 & 0,3 & 8,1 & 0,6 \\
\hline 5876. & 18,1 & 1,2 & 17 & 0,4 & 17,9 & 0,6 & 19,9 & 0,8 \\
\hline 6300. & 33,2 & 1,8 & 0 & 0 & 10,2 & 0,4 & 26,9 & 0,9 \\
\hline 6312. & 0,6 & 0,1 & 1,2 & 0,3 & 2 & 0,2 & 3,7 & 0,5 \\
\hline 6435. & 0 & 0 & 0 & 0 & 0,2 & 0,1 & 0 & 0 \\
\hline 6548. & 149 & 5,4 & 150 & 0,4 & 129 & 1,3 & 188 & 1,7 \\
\hline 6563. & 235 & 7,5 & 285 & 0,4 & 286 & 1,9 & 286 & 2 \\
\hline 6584 & 425 & 11,4 & 432 & 0,4 & 365 & 2,1 & 464 & 2,3 \\
\hline 6678. & 5 & 0,5 & 5,1 & 0,3 & 5,1 & 0,3 & 5,3 & 0,5 \\
\hline 6716. & 12,1 & 0,9 & 53 & 0,4 & 45,7 & 0,8 & 67,6 & 1,2 \\
\hline 6731. & 12,6 & 0,9 & 44,6 & 0,4 & 38,5 & 0,8 & 54,6 & 1,1 \\
\hline 7005 . & 0,2 & 0,2 & 0 & 0 & 0,5 & 0,1 & 1,1 & 0,3 \\
\hline 7065 . & 4,5 & 0,4 & 4,4 & 0,3 & 2,2 & 0,2 & 1,6 & 0,4 \\
\hline 7135 . & 29,2 & 1,6 & 23,3 & 0,4 & 30 & 0,7 & 31,3 & 1 \\
\hline 7237 . & 0,7 & 0,1 & 2 & 0,3 & 1 & 0,2 & 1,6 & 0,4 \\
\hline 7263 . & 0 & 0 & 0 & 0 & 0,2 & 0,1 & 0,8 & 0,3 \\
\hline 7281 & 0,8 & 0,1 & 1,8 & 0,3 & 0,5 & 0,1 & 0,9 & 0,3 \\
\hline 7325 . & 14,5 & 1 & 9,4 & 0,3 & 7,7 & 0,4 & 11,6 & 0,7 \\
\hline 7532 . & 0 & 0 & 0,8 & 0,3 & 0,4 & 0,1 & 0,5 & 0,2 \\
\hline 7751. & 0 & 0 & 0 & 0 & 0 & 0 & 0 & 0 \\
\hline
\end{tabular}

Tabela C.2 - Comparação entre fluxos observados e os fluxos previstos pelo modelo de IC4406. Janelas 1 a 5.

\begin{tabular}{|c|c|c|c|c|c|c|c|c|c|c|}
\hline$\lambda(\stackrel{\circ}{A})$ & Janela 1 & $\sigma$ & Janela 2 & $\sigma$ & Janela 3 & $\sigma$ & Janela 4 & $\sigma$ & Janela 5 & $\sigma$ \\
\hline$\lambda$ & $\mathrm{F}_{H \beta}^{o b s 1}$ & $\log \frac{F_{H \beta}^{o b s 1}}{F_{H \beta}^{m o d}}$ & $\mathrm{~F}_{H \beta}^{o b s 2}$ & $\log \frac{F_{H \beta}^{o b s 2}}{F_{H \beta}^{m o d}}$ & $\mathrm{~F}_{H \beta}^{o b s 3}$ & $\log \frac{F_{H \beta}^{o b s 3}}{F_{H \beta}^{m o d}}$ & $\mathrm{~F}_{H \beta}^{o b s 4}$ & $\log \frac{F_{H \beta}^{o b s 4}}{F_{H \beta}^{m o d}}$ & $\mathrm{~F}_{H \beta}^{o b s 5}$ & $\log \frac{F_{H \beta}^{o b s 5}}{F_{H \beta}^{m o d}}$ \\
\hline 3727. & 880,61 & 0,09 & 1088,09 & $-0,05$ & 893,25 & 0,08 & 711,89 & 0,16 & 529,27 & 0,07 \\
\hline 3798. & 110,58 & 1,31 & 48,11 & 0,96 & 14,34 & 0,43 & 20,69 & 0,59 & 13,54 & 0,41 \\
\hline 3820 . & 53,98 & 1,09 & 22,84 & 1,06 & 22,38 & 1,1 & 15,31 & 0,93 & 0 & - \\
\hline 3835. & 28,2 & 0,58 & 3,37 & $-0,34$ & 2,66 & $-0,44$ & 0 & - & 1,19 & $-0,79$ \\
\hline 3869. & 123,92 & $-0,5$ & 135,19 & $-0,44$ & 106,96 & $-0,47$ & 128,24 & $-0,34$ & 0 & - \\
\hline 3889. & 79,14 & 0,88 & 24,48 & 0,37 & 16,59 & 0,2 & 16,48 & 0,2 & 28,88 & 0,44 \\
\hline 3968. & 64,94 & $-0,26$ & 31,51 & $-0,55$ & 79,81 & $-0,08$ & 54,75 & $-0,19$ & 44,27 & $-0,3$ \\
\hline 4026. & 26,04 & 0,51 & 12,45 & 0,53 & 4,87 & 0,17 & 6,26 & 0,29 & 5,34 & 0,22 \\
\hline 4074. & 8,12 & $-0,31$ & 14 & 0,39 & 8,14 & 0,43 & 2,44 & 0,05 & 2,91 & 0,14 \\
\hline 4102. & 18,54 & $-0,14$ & 21,98 & $-0,07$ & 22,92 & $-0,05$ & 17,83 & $-0,16$ & 30,34 & 0,07 \\
\hline 4267. & 0 & - & 2,16 & 0,64 & 0 & - & 0,46 & $-0,18$ & 0,56 & $-0,1$ \\
\hline 4300 . & 0 & - & 0,42 & $-1,05$ & 0 & - & 0,46 & $-0,52$ & 0 & - \\
\hline
\end{tabular}

Continua na próxima página... 
Tabela C.2 - Continuação

\begin{tabular}{|c|c|c|c|c|c|c|c|c|c|c|}
\hline$\lambda(\stackrel{\circ}{A})$ & Janela 1 & $\sigma$ & Janela 2 & $\sigma$ & Janela 3 & $\sigma$ & Janela 4 & $\sigma$ & Janela 5 & $\sigma$ \\
\hline 4341. & 87,2 & - & 25,92 & 2,41 & 45,3 & 2,36 & 37,36 & 2,27 & 51,34 & 2,23 \\
\hline 4363. & 10,78 & - & 6,11 & 0,46 & 9,31 & 0,37 & 5,34 & 0,12 & 4,49 & $-0,03$ \\
\hline 4471. & 26,66 & 0,19 & 6,93 & $-0,06$ & 5,21 & $-0,13$ & 8,47 & 0,09 & 6,82 & $-0,01$ \\
\hline 4686. & 3,3 & - & 10,62 & - & 3,62 & - & 1,44 & - & 1,37 & 1,14 \\
\hline 4711. & 6,73 & - & 3,55 & 1,55 & 1,77 & 0,65 & 0,52 & $-0,29$ & 0,85 & $-0,3$ \\
\hline 4740. & 3,98 & - & 0,58 & 0,77 & 1,05 & 0,54 & 1,43 & 0,25 & 0,38 & $-0,54$ \\
\hline 4861. & 100 & 0 & 100 & 0 & 100 & 0 & 100 & 0 & 100 & 0 \\
\hline 4922. & 10,5 & 0,35 & 3,42 & 0,21 & 0 & - & 0 & - & 0 & - \\
\hline 4959. & 65,18 & 2,21 & 99,69 & $-0,03$ & 186,02 & $-0,08$ & 284,98 & 0,04 & 303,7 & 0,02 \\
\hline 5007. & 211,36 & 2,21 & 305,68 & $-0,02$ & 583,08 & $-0,07$ & 882,39 & 0,05 & 960,25 & 0,04 \\
\hline 5192. & 0 & - & 0 & - & 12,11 & 1,48 & 0 & - & 0 & - \\
\hline 5199. & 0 & - & 14,93 & $-0,75$ & 12,11 & $-0,39$ & 4,99 & $-0,68$ & 0 & - \\
\hline 5412. & 2,22 & - & 0,38 & - & 0,73 & - & 0,6 & - & 0,07 & - \\
\hline 5518. & 3,48 & 1,06 & 1,96 & 0,39 & 1,47 & 0,21 & 1,79 & 0,3 & 1,75 & 0,29 \\
\hline 5538. & 1,81 & 0,96 & 1,13 & 0,27 & 0,6 & $-0,07$ & 1,1 & 0,26 & 0,45 & $-0,19$ \\
\hline 5755. & 14,76 & 0,06 & 8,79 & $-0,27$ & 9,5 & $-0,01$ & 7,38 & 0,06 & 5,62 & $-0,04$ \\
\hline 5876. & 21,06 & $-0,37$ & 17,68 & $-0,1$ & 20,32 & 0,01 & 17,38 & $-0,05$ & 16,97 & $-0,06$ \\
\hline 6300. & 77,11 & -1 & 49,72 & $-0,39$ & 33,44 & $-0,16$ & 20,05 & $-0,24$ & 20,43 & $-0,17$ \\
\hline 6312. & 3,79 & 1,58 & 1,72 & 0,54 & 4,44 & 0,87 & 1,35 & 0,43 & 0,82 & 0,21 \\
\hline 6435. & 0,55 & - & 0,36 & - & 0 & - & 0,54 & - & 0,06 & - \\
\hline 6548. & 340,39 & $-0,13$ & 237,96 & $-0,23$ & 218,51 & $-0,04$ & 177,35 & 0,04 & 149,11 & 0 \\
\hline 6563. & 284,51 & $-0,03$ & 240,05 & $-0,09$ & 285,39 & $-0,01$ & 284,24 & $-0,01$ & 286,34 & $-0,01$ \\
\hline 6584. & 1025,03 & $-0,12$ & 760,9 & $-0,19$ & 644,25 & $-0,04$ & 522,19 & 0,04 & 432,62 & $-0,01$ \\
\hline 6678. & 6,52 & $-0,33$ & 5,28 & $-0,07$ & 6,33 & 0,05 & 4,8 & $-0,06$ & 4,73 & $-0,06$ \\
\hline 6716. & 122,86 & $-0,11$ & 90,94 & 0,36 & 60,49 & 0,48 & 29,74 & 0,33 & 20,26 & 0,24 \\
\hline 6731. & 90,45 & $-0,11$ & 71,01 & 0,35 & 47,35 & 0,45 & 22,97 & 0,28 & 17,72 & 0,21 \\
\hline 7005 . & 0,86 & - & 0 & - & 0,76 & - & 0,48 & - & 0,5 & - \\
\hline 7065 . & 7,83 & $-0,04$ & 3,55 & $-0,09$ & 3,2 & $-0,11$ & 3,72 & $-0,04$ & 4,41 & $-0,01$ \\
\hline 7135. & 36,48 & $-0,28$ & 23,76 & $-0,46$ & 28,26 & $-0,33$ & 26,74 & $-0,3$ & 18,13 & $-0,47$ \\
\hline 7237. & 12,48 & - & 1,27 & - & 2,95 & - & 2,36 & - & 1,06 & - \\
\hline 7263. & 4,8 & - & 2,47 & - & 2,44 & - & 2,16 & - & 0,78 & - \\
\hline 7281. & 1,72 & $-0,14$ & 1,42 & 0,07 & 4,09 & 0,61 & 0,97 & $-0,01$ & 0,53 & $-0,28$ \\
\hline 7325 . & 17,04 & 0,2 & 14,46 & $-0,28$ & 9,57 & $-0,27$ & 13,22 & 0,02 & 13,61 & 0,01 \\
\hline 7532. & 21,17 & 2,03 & 0 & - & 0,5 & 0 & 0 & - & 0 & - \\
\hline 7751. & 0 & - & 0 & - & 0 & - & 0 & - & 0 & - \\
\hline
\end{tabular}


Tabela C.3 - Comparação entre fluxos observados e os fluxos previstos pelo modelo de IC4406. Janelas 6 a 10.

\begin{tabular}{|c|c|c|c|c|c|c|c|c|c|c|}
\hline$\lambda(\stackrel{\cap}{A})$ & Janela 6 & $\sigma$ & Janela 7 & $\sigma$ & Janela 8 & $\sigma$ & Janela 9 & $\sigma$ & Janela10 & $\sigma$ \\
\hline$\lambda$ & $\mathrm{F}_{H \beta}^{o b s 6}$ & $\log \frac{F_{H \beta}^{o b s 6}}{F_{H \beta}^{m o d}}$ & $\mathrm{~F}_{H \beta}^{o b s 7}$ & $\log \frac{F_{H \beta}^{o b s 7}}{F_{H \beta}^{m o d}}$ & $\mathrm{~F}_{H \beta}^{o b s 8}$ & $\log \frac{F_{H \beta}^{o b s 8}}{F_{H \beta}^{m o d}}$ & $\mathrm{~F}_{H \beta}^{o b s 9}$ & $\log \frac{F_{H \beta}^{o b s 9}}{F_{H \beta}^{m o d}}$ & $\mathrm{~F}_{H \beta}^{o b s 10}$ & $\log \frac{F_{H \beta}^{o b s 10}}{F_{H \beta}^{m o d}}$ \\
\hline 3727. & 438,49 & 0,14 & 369,12 & 0,3 & 345,92 & 0,03 & 437,02 & $-0,02$ & 576,9 & 0,07 \\
\hline 3798. & 4,54 & $-0,07$ & 1,41 & $-0,58$ & 2,48 & $-0,33$ & 3,35 & $-0,2$ & 3,15 & $-0,23$ \\
\hline 3820. & 0,48 & $-0,55$ & 0 & - & 0 & - & 0,39 & $-0,66$ & 3,29 & 0,26 \\
\hline 3835. & 14,6 & 0,3 & 0 & - & 12,63 & 0,24 & 0,39 & $-1,27$ & 1,93 & $-0,58$ \\
\hline 3869. & 0 & - & 0 & - & 0 & - & 0 & - & 0 & - \\
\hline 3889. & 29,23 & 0,45 & 24,7 & 0,37 & 25,74 & 0,39 & 26,06 & 0,4 & 27,85 & 0,42 \\
\hline 3968. & 71,27 & $-0,1$ & 70,58 & $-0,11$ & 67,06 & $-0,13$ & 69,18 & $-0,1$ & 67,21 & $-0,1$ \\
\hline 4026. & 3,68 & 0,07 & 1,4 & $-0,3$ & 4,17 & 0,13 & 3,44 & 0,03 & 4,39 & 0,14 \\
\hline 4074 . & 0 & - & 0 & - & 0 & - & 1,99 & $-0,02$ & 2,94 & 0,13 \\
\hline 4102. & 20,35 & $-0,1$ & 28,92 & 0,05 & 29,75 & 0,06 & 28,66 & 0,05 & 17,4 & $-0,17$ \\
\hline 4267. & 1,36 & 0,35 & 0,64 & 0,03 & 0,87 & 0,16 & 0,3 & $-0,37$ & 0,61 & $-0,06$ \\
\hline 4300. & 0 & - & 0 & - & 0 & - & 0 & - & 0,17 & $-0,95$ \\
\hline 4341. & 50,8 & 2,23 & 47,98 & 2,2 & 50,54 & 2,23 & 50,25 & 2,22 & 33,98 & 2,23 \\
\hline 4363. & 7,23 & 0,09 & 7,97 & 0,06 & 8,18 & 0,14 & 7,08 & 0,17 & 4,84 & 0,07 \\
\hline 4471. & 5,94 & $-0,05$ & 7,27 & 0,08 & 5,83 & $-0,06$ & 5,78 & $-0,08$ & 7,02 & 0,01 \\
\hline 4686. & 9,82 & 0,74 & 26,88 & 0,25 & 22,5 & 1,1 & 4,35 & 1,64 & 0,82 & - \\
\hline 4711. & 1,53 & $-0,34$ & 3,89 & $-0,27$ & 2,78 & $-0,08$ & 1,01 & $-0,23$ & 0,88 & $-0,06$ \\
\hline 4740 . & 0,83 & $-0,5$ & 2,55 & $-0,39$ & 1,53 & $-0,23$ & 0,64 & $-0,31$ & 0,14 & $-0,77$ \\
\hline 4861. & 100 & 0 & 100 & 0 & 100 & 0 & 100 & 0 & 100 & 0 \\
\hline 4922. & 0 & - & 0 & - & 0 & - & 0 & - & 0 & - \\
\hline 4959. & 354,7 & 0,02 & 361,81 & $-0,01$ & 378,43 & 0,05 & 342,96 & 0,08 & 287,77 & 0,04 \\
\hline 5007. & 1068,14 & 0,02 & 1099,22 & $-0,01$ & 1130,89 & 0,05 & 1058,66 & 0,09 & 913,82 & 0,07 \\
\hline 5192. & 0 & - & 0 & - & 0 & - & 0 & - & 0 & - \\
\hline 5199. & 0 & - & 0 & - & 0 & - & 0 & - & 0 & - \\
\hline 5412. & 0,72 & 0,86 & 2,04 & 0,31 & 1,69 & 1,23 & 0,34 & - & 0,47 & - \\
\hline 5518. & 0,51 & $-0,2$ & 0,52 & $-0,06$ & 0,64 & $-0,1$ & 0,68 & $-0,12$ & 0,53 & $-0,23$ \\
\hline 5538. & 0,23 & $-0,49$ & 0,36 & $-0,22$ & 0,36 & $-0,29$ & 0,32 & $-0,34$ & 0,42 & $-0,15$ \\
\hline 5755. & 5,42 & 0,05 & 4,73 & 0,21 & 4,52 & $-0,03$ & 5,3 & $-0,07$ & 7,49 & 0,06 \\
\hline 5876. & 15,47 & $-0,09$ & 13,68 & $-0,1$ & 13,98 & $-0,13$ & 15,63 & $-0,09$ & 16,93 & $-0,06$ \\
\hline 6300. & 18,89 & $-0,04$ & 24,66 & 0,26 & 22,19 & 0,03 & 22,96 & $-0,12$ & 32,81 & $-0,03$ \\
\hline 6312. & 0,28 & $-0,25$ & 0,36 & $-0,05$ & 0,42 & $-0,08$ & 0,65 & 0,12 & 1,3 & 0,41 \\
\hline 6435. & 0,03 & - & 0 & - & 0 & - & 0,02 & - & 0,19 & - \\
\hline 6548. & 125,12 & 0,06 & 107,92 & 0,22 & 107,43 & 0 & 120,2 & $-0,09$ & 179,08 & 0,04 \\
\hline 6563. & 284,09 & $-0,01$ & 284,5 & $-0,01$ & 285,3 & $-0,01$ & 284,37 & $-0,01$ & 284,34 & $-0,01$ \\
\hline 6584. & 360,64 & 0,05 & 310,81 & 0,21 & 312,72 & $-0,01$ & 341,95 & $-0,11$ & 528,4 & 0,04 \\
\hline 6678. & 4,39 & $-0,07$ & 3,74 & $-0,11$ & 3,93 & $-0,12$ & 4,25 & $-0,1$ & 4,96 & $-0,05$ \\
\hline 6716. & 7,87 & 0,02 & 4,55 & 0,02 & 5,07 & $-0,18$ & 13,02 & 0,05 & 28,61 & 0,31 \\
\hline 6731. & 8,28 & 0,01 & 5,13 & 0,01 & 5,3 & $-0,18$ & 12,04 & 0,04 & 23,8 & 0,3 \\
\hline 7005. & 0,02 & - & 0,22 & $-0,5$ & 0,01 & - & 0,07 & - & 0,41 & - \\
\hline 7065 . & 3,79 & $-0,15$ & 3,32 & $-0,24$ & 3,51 & $-0,19$ & 3,76 & $-0,08$ & 4,11 & 0 \\
\hline
\end{tabular}

Continua na próxima página.. . 
Tabela C.3 - Continuação

\begin{tabular}{|c|c|c|c|c|c|c|c|c|c|c|}
\hline$\lambda(\stackrel{\circ}{)}$ & Janela 6 & $\sigma$ & Janela 7 & $\sigma$ & Janela 8 & $\sigma$ & Janela 9 & $\sigma$ & Janela10 & $\sigma$ \\
\hline 7135 & 24,64 & $-0,3$ & 22,57 & $-0,22$ & 23,25 & $-0,32$ & 23,57 & $-0,35$ & 26,93 & $-0,3$ \\
\hline 7237 . & 0,47 & - & 0,55 & 0,74 & 0,75 & - & 0,67 & - & 1,02 & - \\
\hline 7263. & 0,2 & - & 0,15 & 0,19 & 0,13 & - & 0,31 & - & 0,71 & - \\
\hline 7281. & 0,83 & $-0,08$ & 0,74 & $-0,09$ & 0,6 & $-0,22$ & 0,67 & $-0,17$ & 0,07 & $-1,17$ \\
\hline 7325. & 14,54 & 0,09 & 13,45 & 0,22 & 11,96 & 0 & 11,72 & $-0,06$ & 11,38 & $-0,04$ \\
\hline 7532. & 0 & - & 0,01 & $-1,46$ & 0,1 & $-0,61$ & 0,1 & $-0,69$ & 0,08 & $-0,79$ \\
\hline 7751. & 0 & - & 0 & - & 0 & - & 0 & - & 0 & - \\
\hline
\end{tabular}

Tabela C.4 - Comparação entre fluxos observados e os fluxos previstos pelo modelo de IC4406. Janelas 11 a 13.

\begin{tabular}{|c|c|c|c|c|c|c|}
\hline$\lambda(\AA)$ & Janela11 & $\sigma$ & Janela12 & $\sigma$ & Janela13 & $\sigma$ \\
\hline$\lambda$ & $\mathrm{F}_{H \beta}^{o b s 11}$ & $\log \frac{F_{H \beta}^{o b s 11}}{F_{H \beta}^{m o d}}$ & $\mathrm{~F}_{H \beta}^{o b s 12}$ & $\log \frac{F_{H \beta}^{o b s 12}}{F_{H \beta}^{m o d}}$ & $\mathrm{~F}_{H \beta}^{o b s 13}$ & $\log \frac{F_{H \beta}^{o b s 13}}{F_{H \beta}^{m o d}}$ \\
\hline 3727. & 688,8 & $-0,03$ & 654,03 & $-0,27$ & 1083,59 & 0,18 \\
\hline 3798. & 73,62 & 1,14 & 1,44 & $-0,57$ & 16,86 & 0,49 \\
\hline 3820. & 0 & - & 32,19 & 1,21 & 11,46 & 0,42 \\
\hline 3835. & 0 & - & 19,99 & 0,44 & 20,08 & 0,43 \\
\hline 3869. & 146,03 & $-0,34$ & 33,56 & $-1,04$ & 102,9 & $-0,58$ \\
\hline 3889. & 27,83 & 0,42 & 24,69 & 0,37 & 25,38 & 0,38 \\
\hline 3968. & 67,7 & $-0,15$ & 70,31 & $-0,2$ & 37,08 & $-0,51$ \\
\hline 4026. & 9,82 & 0,47 & 4,93 & 0,13 & 4,88 & $-0,22$ \\
\hline 4074. & 2,69 & $-0,05$ & 18,44 & 0,51 & 9,92 & $-0,22$ \\
\hline 4102. & 27,38 & 0,03 & 13,05 & $-0,3$ & 12,44 & $-0,31$ \\
\hline 4267. & 1,15 & 0,28 & 11,3 & 1,35 & 11,18 & 1,75 \\
\hline 4300. & 0,97 & $-0,36$ & 0 & - & 0 & - \\
\hline 4341. & 55,69 & 2,45 & 55,13 & 2,74 & 62,21 & - \\
\hline 4363. & 8,68 & 0,34 & 2,09 & 0 & 4,19 & - \\
\hline 4471. & 8,31 & 0,07 & 3,95 & $-0,3$ & 4,83 & $-0,56$ \\
\hline 4686 . & 1,48 & - & 2,86 & - & 0 & - \\
\hline 4711. & 3,36 & 0,92 & 2,24 & 1,35 & 2,7 & - \\
\hline 4740. & 0,88 & 0,47 & 1,79 & 1,25 & 0 & - \\
\hline 4861. & 100 & 0 & 100 & 0 & 100 & 0 \\
\hline 4922. & 0 & - & 0 & - & 0 & - \\
\hline 4959. & 246,59 & 0,04 & 180,04 & 0,23 & 125,79 & 2,5 \\
\hline 5007. & 742,36 & 0,04 & 560,37 & 0,24 & 382,98 & 2,47 \\
\hline 5192. & 0 & - & 0 & - & 0 & - \\
\hline 5199. & 0 & - & 8,87 & $-0,98$ & 20,07 & $-1,64$ \\
\hline 5412. & 1,14 & - & 1,19 & - & 0 & - \\
\hline 5518. & 0,23 & $-0,59$ & 0,4 & $-0,3$ & 0,61 & 0,31 \\
\hline
\end{tabular}

Continua na próxima página... 
Tabela C.4 - Continuação

\begin{tabular}{|c|c|c|c|c|c|c|}
\hline$\lambda(\stackrel{\circ}{A})$ & Janela11 & $\sigma$ & Janela12 & $\sigma$ & Janela13 & $\sigma$ \\
\hline 5538. & 1,42 & 0,31 & 0,45 & $-0,13$ & 1,22 & 0,79 \\
\hline 5755. & 6,91 & $-0,15$ & 10,05 & $-0,21$ & 9,31 & $-0,14$ \\
\hline 5876. & 14,81 & $-0,13$ & 15,67 & $-0,15$ & 19,66 & $-0,4$ \\
\hline 6300. & 28,05 & $-0,24$ & 36,32 & $-0,52$ & 39,45 & $-1,29$ \\
\hline 6312. & 1,1 & 0,26 & 2,87 & 0,76 & 0,94 & 0,98 \\
\hline 6435. & 0,9 & - & 0,33 & - & 0 & - \\
\hline 6548. & 217,68 & $-0,05$ & 247,03 & $-0,21$ & 268,43 & $-0,23$ \\
\hline 6563. & 278,88 & $-0,02$ & 270,02 & $-0,04$ & 268,72 & $-0,05$ \\
\hline 6584. & 644,22 & $-0,04$ & 736,1 & $-0,21$ & 812,5 & $-0,22$ \\
\hline 6678. & 5,15 & $-0,04$ & 4,6 & $-0,13$ & 5,74 & $-0,39$ \\
\hline 6716. & 59,41 & 0,47 & 74,13 & 0,27 & 95,42 & $-0,22$ \\
\hline 6731. & 46,14 & 0,44 & 57,62 & 0,26 & 72,69 & $-0,21$ \\
\hline 7005. & 0,35 & - & 0 & - & 0,96 & - \\
\hline 7065. & 4,85 & 0,07 & 5,1 & 0,06 & 3,61 & $-0,38$ \\
\hline 7135. & 28,82 & $-0,33$ & 24,6 & $-0,45$ & 29,72 & $-0,37$ \\
\hline 7237. & 1,55 & - & 2 & - & 2,18 & - \\
\hline 7263. & 1,18 & - & 2,23 & - & 1,64 & - \\
\hline 7281. & 0,75 & $-0,13$ & 4,43 & 0,57 & 2,19 & $-0,04$ \\
\hline 7325. & 6,19 & $-0,46$ & 9,06 & $-0,48$ & 20,57 & 0,28 \\
\hline 7532. & 0 & - & 0,61 & 0,18 & 0 & - \\
\hline 7751. & 0 & - & 0 & - & 0 & - \\
\hline
\end{tabular}

Tabela C.5 - Comparação entre fluxos observados e os fluxos previstos pelo modelo de NGC5844 - Fenda Superior ( $\left.{ }^{\circ} 1\right)$. Janelas 1 a 5.

\begin{tabular}{|c|c|c|c|c|c|c|c|c|c|c|}
\hline$\lambda(\stackrel{\circ}{A})$ & Janela 1 & $\sigma$ & Janela 2 & $\sigma$ & Janela 3 & $\sigma$ & Janela 4 & $\sigma$ & Janela 5 & $\sigma$ \\
\hline$\lambda$ & $\mathrm{F}_{H \beta}^{o b s 1}$ & $\log \frac{F_{H \beta}^{o b s 1}}{F_{H \beta}^{m o d}}$ & $\mathrm{~F}_{H \beta}^{o b s 2}$ & $\log \frac{F_{H \beta}^{o b s 2}}{F_{H \beta}^{m o d}}$ & $\mathrm{~F}_{H \beta}^{o b s 3}$ & $\log \frac{F_{H \beta}^{o b s 3}}{F_{H \beta}^{m o d}}$ & $\mathrm{~F}_{H \beta}^{o b s 4}$ & $\log \frac{F_{H \beta}^{o b s 4}}{F_{H \beta}^{m o d}}$ & $\mathrm{~F}_{H \beta}^{o b s 5}$ & $\log \frac{F_{H \beta}^{o b s 5}}{F_{H \beta}^{m o d}}$ \\
\hline 3727. & 203,56 & $-0,25$ & 140,66 & 0,01 & 176,35 & 0,24 & 60,36 & $-0,23$ & 262,83 & 0,41 \\
\hline 3798. & 22,65 & 0,63 & 50,34 & 0,98 & 23,86 & 0,65 & 16,89 & 0,5 & 20,73 & 0,58 \\
\hline 3820. & 23,83 & 1,08 & 15 & 0,9 & 0 & - & 0 & - & 0 & - \\
\hline 3835. & 0 & - & 15,41 & 0,32 & 14,97 & 0,31 & 0 & - & 765,52 & 2,02 \\
\hline 3869. & 136,43 & $-0,25$ & 137,13 & $-0,09$ & 159,35 & $-0,02$ & 131,61 & $-0,14$ & 192,09 & 0,03 \\
\hline 3889. & 44,52 & 0,63 & 33,31 & 0,51 & 16,22 & 0,19 & 29,14 & 0,44 & 0 & - \\
\hline 3968. & 58,7 & $-0,1$ & 0 & - & 82,96 & 0,22 & 69,68 & 0,11 & 0 & - \\
\hline 4026. & 8,44 & 0,36 & 12,21 & 0,53 & 0 & - & 2,84 & $-0,04$ & 2,07 & $-0,18$ \\
\hline 4074. & 0 & - & 4,98 & 0,18 & 13,71 & 0,76 & 8,11 & 0,53 & 9,83 & 0,61 \\
\hline 4102. & 22,14 & $-0,07$ & 28,3 & 0,04 & 42,63 & 0,22 & 34,26 & 0,12 & 27,4 & 0,03 \\
\hline 4267. & 2,66 & 0,58 & 2,05 & 0,47 & 3,18 & 0,66 & 3,06 & 0,71 & 1,33 & 0,35 \\
\hline 4300. & 0 & - & 1,05 & 0,55 & 0 & - & 0 & - & 1,19 & 0,78 \\
\hline
\end{tabular}

Continua na próxima página... 
Tabela C.5 - Continuação

\begin{tabular}{|c|c|c|c|c|c|c|c|c|c|c|}
\hline$\lambda(\stackrel{\circ}{A})$ & Janela 1 & $\sigma$ & Janela 2 & $\sigma$ & Janela 3 & $\sigma$ & Janela 4 & $\sigma$ & Janela 5 & $\sigma$ \\
\hline 4341. & 44,64 & 2,17 & 49,32 & 2,09 & 70,42 & 2,25 & 49,96 & 2,1 & 48,88 & 2,09 \\
\hline 4363. & 3,06 & 0,12 & 2,43 & 0,18 & 11,47 & 0,83 & 3,99 & 0,28 & 3,06 & 0,16 \\
\hline 4471. & 1,71 & $-0,66$ & 6,45 & $-0,08$ & 7,03 & $-0,03$ & 5,22 & $-0,11$ & 5,97 & $-0,05$ \\
\hline 4686. & 3,6 & - & 9,83 & - & 28,07 & 1,02 & 22,4 & 0,01 & 26,64 & 0,08 \\
\hline 4711. & 1,89 & 0,43 & 0,38 & $-0,65$ & 1,56 & $-0,36$ & 1,59 & $-0,54$ & 0,93 & $-0,77$ \\
\hline 4740. & 1,39 & 0,44 & 0,76 & $-0,23$ & 5,03 & 0,27 & 1,91 & $-0,33$ & 0,28 & $-1,16$ \\
\hline 4861. & 100 & 0 & 100 & 0 & 100 & 0 & 100 & 0 & 100 & 0 \\
\hline 4922. & 0 & - & 0 & - & 1,38 & $-0,16$ & 1,11 & $-0,21$ & 0,64 & $-0,45$ \\
\hline 4959. & 291,54 & 0,11 & 282,64 & 0,12 & 239,81 & 0,03 & 237,33 & 0,03 & 239,69 & 0,03 \\
\hline 5007. & 911,59 & 0,12 & 909,43 & 0,15 & 597,95 & $-0,05$ & 730,36 & 0,04 & 720,72 & 0,03 \\
\hline 5192. & 3,26 & 1,21 & 0,94 & 0,98 & 0 & - & 0 & - & 0 & - \\
\hline 5199. & 3,25 & $-0,73$ & 0,94 & $-0,88$ & 0 & - & 0 & - & 0 & - \\
\hline 5412. & 0,63 & - & 1,73 & - & 0 & - & 4,11 & 0,47 & 2,77 & 0,3 \\
\hline 5518. & 1,32 & 0,34 & 1,61 & 0,61 & 1,88 & 0,8 & 0,47 & 0,2 & 0,98 & 0,51 \\
\hline 5538. & 0,69 & 0,24 & 0,61 & 0,31 & 0 & - & 0,33 & 0,21 & 0,99 & 0,69 \\
\hline 5755. & 10,03 & 0,2 & 2,82 & 0,07 & 4,64 & 0,41 & 4,99 & 0,44 & 6,72 & 0,57 \\
\hline 5876. & 19,78 & $-0,05$ & 13,36 & $-0,22$ & 17,7 & $-0,08$ & 17,25 & $-0,05$ & 17,35 & $-0,05$ \\
\hline 6300. & 14,61 & $-0,14$ & 3,55 & $-0,36$ & 15,63 & 0,41 & 0 & - & 19 & 0,49 \\
\hline 6312. & 1,44 & $-0,19$ & 1,06 & $-0,12$ & 1,31 & 0,12 & 0,65 & $-0,19$ & 1,01 & 0,01 \\
\hline 6435. & 0,59 & - & 0,27 & - & 0 & - & 0 & - & 0,23 & $-0,24$ \\
\hline 6548. & 186,02 & $-0,08$ & 81,09 & $-0,03$ & 152,1 & 0,38 & 146,98 & 0,37 & 123,93 & 0,29 \\
\hline 6563. & 285,06 & $-0,02$ & 284,28 & $-0,02$ & 289,21 & $-0,01$ & 285,36 & $-0,02$ & 285,81 & $-0,01$ \\
\hline 6584. & 565,74 & $-0,07$ & 229 & $-0,05$ & 439,5 & 0,38 & 431,8 & 0,37 & 345,1 & 0,27 \\
\hline 6678 & 5,6 & $-0,05$ & 3,91 & $-0,2$ & 5,4 & $-0,05$ & 4,31 & $-0,11$ & 4,93 & $-0,05$ \\
\hline 6716. & 64,55 & 0,01 & 30,85 & 0,08 & 52,05 & 0,45 & 52,28 & 0,45 & 45,24 & 0,39 \\
\hline 6731. & 51,87 & $-0,02$ & 23,59 & 0,03 & 42,88 & 0,43 & 45,14 & 0,45 & 39,3 & 0,39 \\
\hline 7005 & 0 & - & 0,49 & - & 0,36 & - & 0,43 & $-0,27$ & 0,49 & $-0,21$ \\
\hline 7065 & 0 & - & 2,17 & $-0,35$ & 1,68 & $-0,44$ & 1,72 & $-0,39$ & 2,59 & $-0,21$ \\
\hline 7135 & 34,5 & $-0,2$ & 13,83 & $-0,43$ & 23,75 & $-0,09$ & 20,5 & $-0,13$ & 17,88 & $-0,19$ \\
\hline 7237 & 3,44 & - & 2,28 & - & 2,42 & - & 0,93 & - & 0,94 & - \\
\hline 7263 & 2,6 & - & 1,49 & - & 0,49 & - & 1,01 & - & 0,8 & - \\
\hline 7281 & 2,2 & 0,3 & 1,11 & 0,04 & 0 & - & 1,16 & 0,11 & 1,99 & 0,34 \\
\hline 7325 . & 4,26 & $-0,21$ & 4,04 & 0,14 & 11,69 & 0,73 & 8,36 & 0,58 & 8,02 & 0,56 \\
\hline 7532 . & 0 & - & 0 & - & 0 & - & 0 & - & 0 & - \\
\hline 7751 & 0 & - & 0 & - & 0 & - & 0 & - & 0 & - \\
\hline
\end{tabular}


Tabela C.6 - Comparação entre fluxos observados e os fluxos previstos pelo modelo de NGC5844 - Fenda Superior (n $\left.{ }^{\circ} 1\right)$. Janelas 6 a 8.

\begin{tabular}{|c|c|c|c|c|c|c|}
\hline$\lambda(\AA)$ & Janela 1 & $\sigma$ & Janela 2 & $\sigma$ & Janela 3 & $\sigma$ \\
\hline$\lambda$ & $\mathrm{F}_{H \beta}^{o b s 6}$ & $\log \frac{F_{H \beta}^{o b s 6}}{F_{H \beta}^{m o d}}$ & $\mathrm{~F}_{H \beta}^{o b s 7}$ & $\log \frac{F_{H \beta}^{o b b s}}{F_{H \beta}^{m o d}}$ & $\mathrm{~F}_{H \beta}^{o b s s}$ & $\log \frac{F_{H \beta}^{o b s} 8}{F_{H \beta}^{m o d}}$ \\
\hline 3727. & 226,31 & 0,35 & 352,09 & 0,4 & 682,43 & 0,28 \\
\hline 3798. & 22,59 & 0,63 & 16,78 & 0,5 & 14,6 & 0,44 \\
\hline 3820. & 12,55 & 0,82 & 0 & - & 12,98 & 0,81 \\
\hline 3835. & 0 & - & 0 & - & 0 & - \\
\hline 3869. & 145,96 & $-0,05$ & 0 & - & 0 & - \\
\hline 3889. & 10,93 & 0,02 & 20,77 & 0,3 & 37,3 & 0,56 \\
\hline 3968. & 67,05 & 0,13 & 65,95 & 0,12 & 80,21 & 0,04 \\
\hline 4026. & 3,11 & $-0,05$ & 3,1 & $-0,07$ & 0 & - \\
\hline 4074. & 7,15 & 0,47 & 8,22 & 0,4 & 18,67 & 0,36 \\
\hline 4102. & 29,27 & 0,06 & 28,63 & 0,05 & 27,83 & 0,04 \\
\hline 4267. & 1,75 & 0,4 & 0,83 & 0,07 & 0 & - \\
\hline 4300. & 0 & - & 0,51 & 0,23 & 0,91 & 0,11 \\
\hline 4341. & 50,16 & 2,1 & 47,36 & 2,07 & 50,6 & 2,23 \\
\hline 4363. & 3,65 & 0,33 & 2,21 & 0,14 & 2,19 & $-0,02$ \\
\hline 4471. & 4,17 & $-0,26$ & 6,66 & $-0,06$ & 8,34 & 0,02 \\
\hline 4686. & 25,92 & 0,98 & 8,46 & - & 0,79 & - \\
\hline 4711. & 1,01 & $-0,55$ & 0,38 & $-0,65$ & 0,7 & 0 \\
\hline 4740. & 0,41 & $-0,82$ & 1,05 & $-0,09$ & 0,51 & 0,01 \\
\hline 4861. & 100 & 0 & 100 & 0 & 100 & 0 \\
\hline 4922. & 0 & - & 1,65 & $-0,1$ & 0,49 & $-0,63$ \\
\hline 4959. & 272,05 & 0,09 & 275,61 & 0,11 & 184,09 & $-0,09$ \\
\hline 5007. & 843,55 & 0,1 & 870,83 & 0,13 & 550,44 & $-0,1$ \\
\hline 5192. & 0 & - & 0 & - & 0 & - \\
\hline 5199. & 2,13 & $-0,4$ & 3,43 & $-0,32$ & 0 & - \\
\hline 5412. & 3,8 & 1,28 & 0,62 & - & 0,34 & - \\
\hline 5518. & 0,34 & 0,05 & 1,05 & 0,42 & 0,31 & $-0,29$ \\
\hline 5538. & 0,25 & $-0,08$ & 0,75 & 0,4 & 0,33 & $-0,08$ \\
\hline 5755. & 3,44 & 0,28 & 5,83 & 0,39 & 14,9 & 0,37 \\
\hline 5876. & 14,39 & $-0,17$ & 15,83 & $-0,15$ & 20,06 & $-0,05$ \\
\hline 6300. & 10,48 & 0,24 & 17,06 & 0,32 & 70,99 & 0,54 \\
\hline 6312. & 1,27 & 0,11 & 1,2 & $-0,07$ & 1,75 & $-0,1$ \\
\hline 6435. & 0 & - & 0 & - & 0 & - \\
\hline 6548. & 86,4 & 0,14 & 153,94 & 0,25 & 326,66 & 0,16 \\
\hline 6563. & 284,22 & $-0,02$ & 285,14 & $-0,02$ & 284,69 & $-0,02$ \\
\hline 6584. & 237,52 & 0,11 & 450,93 & 0,25 & 1003,19 & 0,18 \\
\hline 6678. & 4,54 & $-0,13$ & 4,74 & $-0,12$ & 5,38 & $-0,07$ \\
\hline 6716. & 31,98 & 0,24 & 52,72 & 0,31 & 127,8 & 0,31 \\
\hline 6731. & 26,35 & 0,22 & 45,43 & 0,31 & 107,97 & 0,3 \\
\hline 7005 . & 0,2 & - & 0 & - & 0,81 & - \\
\hline 7065. & 1,88 & $-0,39$ & 2,9 & $-0,22$ & 4,07 & $-0,08$ \\
\hline
\end{tabular}

Continua na próxima página... 
Tabela C.6 - Continuação

\begin{tabular}{ccccccc}
\hline$\lambda(\AA)$ & Janela 1 & $\sigma$ & Janela 2 & $\sigma$ & Janela 3 & $\sigma$ \\
\hline 7135. & 18,64 & $-0,2$ & 24,16 & $-0,19$ & 18,49 & $-0,47$ \\
7237. & 1 & - & 0,52 & - & 0,41 & - \\
7263. & 0 & - & 0 & - & 0 & - \\
7281. & 1,79 & 0,25 & 0,75 & $-0,13$ & 0,43 & $-0,41$ \\
7325. & 2,72 & 0,09 & 8,91 & 0,49 & 16,64 & 0,38 \\
7532. & 0 & - & 0,4 & 0,3 & 0 & - \\
7751. & 0 & - & 0 & - & 0 & - \\
\hline
\end{tabular}

Tabela C.7 - Comparação entre fluxos observados e os fluxos previstos pelo modelo de NGC5844 - Fenda Central (n $\left.{ }^{\circ} 2\right)$. Janelas 1 a 5.

\begin{tabular}{|c|c|c|c|c|c|c|c|c|c|c|}
\hline$\lambda(\AA)$ & Janela 1 & $\sigma$ & Janela 2 & $\sigma$ & Janela 3 & $\sigma$ & Janela 4 & $\sigma$ & Janela 5 & $\sigma$ \\
\hline$\lambda$ & $\mathrm{F}_{H \beta}^{o b s 1}$ & $\log \frac{F_{H \beta}^{o b s 1}}{F_{H \beta}^{m o d}}$ & $\mathrm{~F}_{H \beta}^{o b s 2}$ & $\log \frac{F_{H \beta}^{o b s 2}}{F_{H \beta}^{m o d}}$ & $\mathrm{~F}_{H \beta}^{o b s 3}$ & $\log \frac{F_{H \beta}^{o b s 3}}{F_{H \beta}^{m o d}}$ & $\mathrm{~F}_{H \beta}^{o b s 4}$ & $\log \frac{F_{H \beta}^{o b s 4}}{F_{H \beta}^{m o d}}$ & $\mathrm{~F}_{H \beta}^{o b s 5}$ & $\log \frac{F_{H \beta}^{o b s} 5}{F_{H \beta}^{m o d}}$ \\
\hline 3727. & 298,86 & $-0,56$ & 327,58 & $-0,05$ & 245,43 & 0,25 & 288,83 & 0,47 & 242,55 & 0,44 \\
\hline 3798. & 106,11 & 1,3 & 30,93 & 0,77 & 38,79 & 0,86 & 62,51 & 1,07 & 4,14 & $-0,12$ \\
\hline 3820. & 70,16 & 1,43 & 0 & 0 & 8,55 & 0,65 & 0 & 0 & 0 & 0 \\
\hline 3835. & 0 & 0 & 0,43 & $-1,23$ & 8,49 & 0,07 & 35,51 & 0,69 & 0 & 0 \\
\hline 3869. & 0 & 0 & 129,32 & $-0,28$ & 108,83 & $-0,19$ & 109,29 & $-0,18$ & 123,1 & $-0,17$ \\
\hline 3889. & 0 & 0 & 40,45 & 0,59 & 37,93 & 0,56 & 11,91 & 0,06 & 30,49 & 0,46 \\
\hline 3968. & 52,11 & $-0,35$ & 54,11 & $-0,14$ & 60,2 & 0,08 & 60,06 & 0,08 & 67,29 & 0,09 \\
\hline 4026. & 16,71 & 0,54 & 3,57 & $-0,02$ & 10,62 & 0,47 & 2,85 & $-0,09$ & 8,98 & 0,51 \\
\hline 4074. & 27,85 & $-0,11$ & 0 & 0 & 0 & 0 & 24,86 & 1,03 & 14,32 & 0,83 \\
\hline 4102. & 13,78 & $-0,27$ & 31,15 & 0,08 & 29,97 & 0,07 & 35,85 & 0,15 & 41,81 & 0,21 \\
\hline 4267. & 0 & 0 & 3,04 & 0,64 & 3,6 & 0,71 & 4,96 & 0,85 & 3,63 & 0,78 \\
\hline 4300. & 0 & 0 & 2,47 & 0,49 & 1,44 & 0,68 & 0 & 0 & 0,84 & 0,62 \\
\hline 4341. & 70,82 & 2,85 & 51,13 & 2,23 & 57,26 & 2,16 & 0 & 0 & 51,42 & 2,11 \\
\hline 4363. & 2,59 & 0,57 & 4,35 & 0,28 & 3,13 & 0,29 & 3,6 & 0,33 & 5,11 & 0,33 \\
\hline 4471. & 6,6 & $-0,19$ & 4,28 & $-0,27$ & 2,85 & $-0,43$ & 4,68 & $-0,21$ & 4,73 & $-0,11$ \\
\hline 4686. & 1,62 & 0 & 3,75 & 0 & 20,19 & 0 & 36,2 & 1,13 & 35,78 & $-0,01$ \\
\hline 4711. & 0,77 & 0 & 0,66 & $-0,03$ & 2,18 & 0,08 & 3,45 & $-0,04$ & 2,15 & $-0,51$ \\
\hline 4740 & 2,06 & 0 & 1,27 & 0,4 & 1,78 & 0,14 & 2,02 & $-0,16$ & 1,28 & $-0,61$ \\
\hline 4861. & 100 & 0 & 100 & 0 & 100 & 0 & 100 & 0 & 100 & 0 \\
\hline 4922. & 0 & 0 & 0 & 0 & 0 & 0 & 0 & 0 & 0 & 0 \\
\hline 4959. & 327 & 0,82 & 303,55 & 0,12 & 298,8 & 0,15 & 285,36 & 0,1 & 270,58 & 0,1 \\
\hline 5007. & 970,58 & 0,81 & 906,98 & 0,12 & 916,97 & 0,15 & 864,74 & 0,11 & 833,59 & 0,11 \\
\hline 5192. & 0 & 0 & 0 & 0 & 0 & 0 & 0 & 0 & 0 & 0 \\
\hline 5199. & 0 & 0 & 0 & 0 & 0 & 0 & 0 & 0 & 2,08 & $-0,35$ \\
\hline 5412. & 0,99 & 0 & 0,1 & 0 & 1,49 & 0 & 2,46 & 1,09 & 2,13 & $-0,05$ \\
\hline 5518. & 2,32 & 0,67 & 0,52 & $-0,07$ & 0,86 & 0,33 & 1,65 & 0,74 & 0,62 & 0,32 \\
\hline
\end{tabular}

Continua na próxima página... 
Tabela C.7 - Continuação

\begin{tabular}{|c|c|c|c|c|c|c|c|c|c|c|}
\hline$\lambda(\stackrel{\circ}{A})$ & Janela 1 & $\sigma$ & Janela 2 & $\sigma$ & Janela 3 & $\sigma$ & Janela 4 & $\sigma$ & Janela 5 & $\sigma$ \\
\hline 5538. & 0,89 & 0,35 & 0,92 & 0,36 & 0,36 & 0,08 & 0 & 0 & 0,45 & 0,36 \\
\hline 5755. & 10,57 & $-0,31$ & 5,12 & $-0,1$ & 3,29 & 0,14 & 1,65 & $-0,01$ & 2,79 & 0,24 \\
\hline 5876. & 18,82 & $-0,19$ & 16,35 & $-0,14$ & 13,29 & $-0,22$ & 10,8 & $-0,3$ & 14,7 & $-0,08$ \\
\hline 6300. & 0 & 0 & 4,46 & $-0,67$ & 3,67 & $-0,33$ & 5,6 & 0 & 5,23 & $-0,01$ \\
\hline 6312. & 2,52 & 0,12 & 2,31 & 0,02 & 1,72 & 0,09 & 1,25 & 0,1 & 2 & 0,35 \\
\hline 6435. & 1,5 & 0 & 0 & 0 & 0,43 & 0 & 0 & 0 & 0 & 0 \\
\hline 6548. & 253,68 & $-0,46$ & 134,53 & $-0,23$ & 105,18 & 0,09 & 63,7 & 0,03 & 82,98 & 0,19 \\
\hline 6563. & 288,83 & $-0,01$ & 286,08 & $-0,01$ & 284,99 & $-0,02$ & 286,35 & $-0,02$ & 285,64 & $-0,01$ \\
\hline 6584 & 780,42 & $-0,44$ & 365,41 & $-0,27$ & 299,32 & 0,07 & 176,9 & 0 & 231,23 & 0,16 \\
\hline 6678. & 5,79 & $-0,15$ & 4,76 & $-0,12$ & 3,88 & $-0,2$ & 2,3 & $-0,42$ & 3,95 & $-0,1$ \\
\hline 6716. & 76,31 & $-0,57$ & 45,5 & $-0,15$ & 36,08 & 0,15 & 19,99 & 0,06 & 27,54 & 0,24 \\
\hline 6731. & 65,07 & $-0,56$ & 36,42 & $-0,18$ & 29,14 & 0,13 & 17,1 & 0,05 & 22,74 & 0,22 \\
\hline 7005 & 0 & 0 & 0,14 & 0 & 0,49 & 0 & 0,05 & 0 & 0,49 & $-0,69$ \\
\hline 7065 & 0,77 & $-0,92$ & 1,37 & $-0,55$ & 1,49 & $-0,52$ & 0,85 & $-0,74$ & 1,27 & $-0,48$ \\
\hline 7135. & 36,59 & $-0,32$ & 28,81 & $-0,28$ & 25,49 & $-0,16$ & 13,67 & $-0,32$ & 20,76 & $-0,07$ \\
\hline 7237 . & 7,23 & 0 & 1,02 & 0 & 0,45 & 0 & 0,39 & 0 & 0,64 & 0,8 \\
\hline 7263 & 3,99 & 0 & 0,57 & 0 & 0,22 & 0 & 0,24 & 0 & 0,16 & 0,21 \\
\hline 7281. & 4,43 & 0,5 & 0,6 & $-0,26$ & 0,36 & $-0,45$ & 0,23 & $-0,63$ & 0,19 & $-0,64$ \\
\hline 7325 & 8,72 & $-0,37$ & 5,72 & $-0,09$ & 5,25 & 0,27 & 2,58 & 0,07 & 5,53 & 0,46 \\
\hline 7532 & 0 & 0 & 0 & 0 & 0 & 0 & 0 & 0 & 0,12 & $-0,23$ \\
\hline 7751. & 0 & 0 & 0 & 0 & 0 & 0 & 0 & 0 & 0 & 0 \\
\hline
\end{tabular}

Tabela C.8 - Comparação entre fluxos observados e os fluxos previstos pelo modelo de NGC5844 - Fenda Central (nº 2). Janelas 6 a 10.

\begin{tabular}{|c|c|c|c|c|c|c|c|c|c|c|}
\hline$\lambda(\stackrel{\cap}{A})$ & Janela 6 & $\sigma$ & Janela 7 & $\sigma$ & Janela 8 & $\sigma$ & Janela 9 & $\sigma$ & Janela10 & $\sigma$ \\
\hline$\lambda$ & $\mathrm{F}_{H \beta}^{o b s 6}$ & $\log \frac{F_{H \beta}^{o b s 6}}{F_{H \beta}^{m o d}}$ & $\mathrm{~F}_{H \beta}^{o b s 7}$ & $\log \frac{F_{H \beta}^{o b s 7}}{F_{H \beta}^{m o d}}$ & $\mathrm{~F}_{H \beta}^{o b s 8}$ & $\log \frac{F_{H \beta}^{o b s 8}}{F_{H \beta}^{m o d}}$ & $\mathrm{~F}_{H \beta}^{o b s 9}$ & $\log \frac{F_{H \beta}^{o b s 9}}{F_{H \beta}^{m o d}}$ & $\mathrm{~F}_{H \beta}^{o b s 10}$ & $\log \frac{F_{H \beta}^{o b s 10}}{F_{H \beta}^{m o d}}$ \\
\hline 3727. & 228,26 & 0,42 & 173,86 & 0,25 & 217,68 & 0,2 & 278,31 & $-0,12$ & 416,24 & $-0,42$ \\
\hline 3798. & 15,72 & 0,46 & 22,13 & 0,62 & 0 & 0 & 0,18 & $-1,48$ & 0 & 0 \\
\hline 3820 . & 8 & 0,7 & 11,77 & 0,79 & 10,3 & 0,73 & 4,82 & 0,38 & 28,27 & 1,04 \\
\hline 3835. & 0 & 0 & 13,97 & 0,28 & 4,71 & $-0,19$ & 0 & 0 & 94,79 & 1,11 \\
\hline 3869. & 144,59 & $-0,1$ & 112,21 & $-0,17$ & 153,67 & $-0,04$ & 174,48 & $-0,15$ & 0 & 0 \\
\hline 3889. & 21,6 & 0,31 & 22,41 & 0,33 & 20,32 & 0,29 & 4,08 & $-0,41$ & 375,09 & 1,55 \\
\hline 3968. & 60,51 & 0,05 & 54,91 & 0,04 & 56,65 & 0,05 & 58,45 & $-0,11$ & 90,48 & $-0,11$ \\
\hline 4026. & 0 & 0 & 3,36 & $-0,02$ & 0 & 0 & 0,86 & $-0,63$ & 0 & 0 \\
\hline 4074 . & 14 & 0,82 & 3,47 & 0,18 & 11 & 0,54 & 1,58 & $-0,73$ & 21,43 & $-0,22$ \\
\hline 4102. & 33,68 & 0,11 & 31,78 & 0,09 & 25,22 & $-0,01$ & 25,37 & $-0,01$ & 29,91 & 0,06 \\
\hline 4267. & 1,62 & 0,43 & 1,49 & 0,33 & 2,05 & 0,47 & 1,32 & 0,27 & 3,44 & 0,84 \\
\hline 4300. & 1,45 & 0,86 & 0 & 0 & 0 & 0 & 0 & 0 & 0 & 0 \\
\hline
\end{tabular}

Continua na próxima página.. . 
Tabela C.8 - Continuação

\begin{tabular}{|c|c|c|c|c|c|c|c|c|c|c|}
\hline$\lambda(\stackrel{\circ}{A})$ & Janela 6 & $\sigma$ & Janela 7 & $\sigma$ & Janela 8 & $\sigma$ & Janela 9 & $\sigma$ & Janela10 & $\sigma$ \\
\hline 4341. & 50,57 & 2,1 & 48,61 & 2,09 & 50,38 & 2,1 & 42,41 & 2,15 & 54,36 & 2,74 \\
\hline 4363. & 3,86 & 0,21 & 3,39 & 0,3 & 4,08 & 0,41 & 1,43 & $-0,21$ & 6,43 & 0,96 \\
\hline 4471. & 5,68 & $-0,03$ & 4,31 & $-0,24$ & 3,6 & $-0,33$ & 5,83 & $-0,13$ & 0 & 0 \\
\hline 4686. & 32,02 & $-0,06$ & 31,86 & 1,07 & 17,3 & 0 & 3,73 & 0 & 0 & 0 \\
\hline 4711. & 1,21 & $-0,76$ & 1,25 & $-0,48$ & 1,55 & $-0,07$ & 1,06 & 0,18 & 0,59 & 0 \\
\hline 4740. & 0,76 & $-0,83$ & 0,72 & $-0,6$ & 1,45 & 0,05 & 1,1 & 0,34 & 0,4 & 0 \\
\hline 4861. & 100 & 0 & 100 & 0 & 100 & 0 & 100 & 0 & 100 & 0 \\
\hline 4922. & 0 & 0 & 0 & 0 & 0 & 0 & 1,32 & $-0,2$ & 2,11 & $-0,12$ \\
\hline 4959. & 262,67 & 0,08 & 274,88 & 0,09 & 291,93 & 0,14 & 305,23 & 0,13 & 310,07 & 0,79 \\
\hline 5007. & 780,32 & 0,08 & 842,67 & 0,1 & 899,05 & 0,15 & 920,68 & 0,13 & 849,64 & 0,75 \\
\hline 5192. & 0 & 0 & 2,25 & 1,35 & 0 & 0 & 3,07 & 1,19 & 14,4 & 1,56 \\
\hline 5199. & 0 & 0 & 2,25 & $-0,34$ & 0 & 0 & 5,37 & $-0,51$ & 14,37 & $-1,05$ \\
\hline 5412. & 2,75 & 0,06 & 2,29 & 1,06 & 1,09 & 0 & 0 & 0 & 0 & 0 \\
\hline 5518. & 0,42 & 0,15 & 0,45 & 0,17 & 0,82 & 0,31 & 1,66 & 0,44 & 10,26 & 1,31 \\
\hline 5538. & 0,36 & 0,26 & 0,59 & 0,29 & 0,4 & 0,12 & 1,06 & 0,42 & 0,28 & $-0,16$ \\
\hline 5755. & 6,02 & 0,58 & 2,43 & 0,16 & 5,36 & 0,35 & 6,85 & 0,02 & 8,33 & $-0,41$ \\
\hline 5876. & 16,64 & $-0,02$ & 14,62 & $-0,17$ & 16,25 & $-0,13$ & 19,27 & $-0,07$ & 14,26 & $-0,31$ \\
\hline 6300. & 17,69 & 0,52 & 6,94 & 0,09 & 14,31 & 0,26 & 19,43 & $-0,03$ & 26,02 & $-0,81$ \\
\hline 6312. & 1,85 & 0,31 & 2,45 & 0,39 & 1,69 & 0,08 & 3,44 & 0,19 & 1,79 & $-0,03$ \\
\hline 6435. & 0,1 & $-1,04$ & 0,44 & 0 & 0,16 & 0 & 0,26 & 0 & 0 & 0 \\
\hline 6548. & 136,52 & 0,4 & 89,14 & 0,18 & 125,37 & 0,17 & 214,96 & $-0,03$ & 297,58 & $-0,39$ \\
\hline 6563. & 286,5 & $-0,01$ & 284,44 & $-0,02$ & 284,94 & $-0,02$ & 283,55 & $-0,02$ & 284,99 & $-0,02$ \\
\hline 6584. & 403,67 & 0,41 & 247,29 & 0,15 & 349,42 & 0,14 & 602,12 & $-0,05$ & 902,16 & $-0,38$ \\
\hline 6678. & 4,86 & $-0,01$ & 4,57 & $-0,13$ & 5,13 & $-0,08$ & 5,67 & $-0,05$ & 4,96 & $-0,22$ \\
\hline 6716. & 46,26 & 0,46 & 30,65 & 0,24 & 43,66 & 0,24 & 68,76 & 0,03 & 84,34 & $-0,53$ \\
\hline 6731. & 40,58 & 0,47 & 26,3 & 0,24 & 37,11 & 0,23 & 55,81 & 0,01 & 67,23 & $-0,54$ \\
\hline 7005 . & 0,42 & $-0,76$ & 0 & 0 & 0 & 0 & 0 & 0 & 0,58 & 0 \\
\hline 7065. & 2,36 & $-0,21$ & 1,99 & $-0,37$ & 1,22 & $-0,61$ & 3,48 & $-0,15$ & 1,95 & $-0,51$ \\
\hline 7135. & 23,42 & $-0,01$ & 22,29 & $-0,11$ & 22,53 & $-0,22$ & 36,73 & $-0,17$ & 33,22 & $-0,36$ \\
\hline 7237. & 1,29 & 1,11 & 0,86 & 0 & 0,73 & 0 & 0,77 & 0 & 0 & 0 \\
\hline 7263. & 0,21 & 0,32 & 0 & 0 & 0,41 & 0 & 0,18 & 0 & 1,71 & 0 \\
\hline 7281. & 1,06 & 0,12 & 0,56 & $-0,25$ & 0,55 & $-0,26$ & 1,12 & 0,01 & 1,88 & 0,13 \\
\hline 7325. & 8,78 & 0,67 & 5,79 & 0,42 & 6,94 & 0,39 & 8,51 & 0,08 & 11,44 & $-0,25$ \\
\hline 7532 . & 0,29 & 0,16 & 0 & 0 & 0 & 0 & 1,28 & 0,63 & 0 & 0 \\
\hline 7751. & 0 & 0 & 0 & 0 & 0 & 0 & 0 & 0 & 0 & 0 \\
\hline
\end{tabular}


Tabela C.9 - Comparação entre fluxos observados e os fluxos previstos pelo modelo de NGC5844 - Fenda Inferior (nº 3$)$. Janelas 1 a 5.

\begin{tabular}{|c|c|c|c|c|c|c|c|c|c|c|}
\hline$\lambda(\AA)$ & Janela 1 & $\sigma$ & Janela 2 & $\sigma$ & Janela 3 & $\sigma$ & Janela 4 & $\sigma$ & Janela 5 & $\sigma$ \\
\hline$\lambda$ & $\mathrm{F}_{H \beta}^{o b s 1}$ & $\log \frac{F_{H \beta}^{o b s} 1}{F_{H \beta}^{m o d}}$ & $\mathrm{~F}_{H \beta}^{o b s 2}$ & $\log \frac{F_{H \beta}^{o b s 2}}{F_{H \beta}^{m o d}}$ & $\mathrm{~F}_{H \beta}^{o b s 3}$ & $\log \frac{F_{H \beta}^{o b s} 3}{F_{H \beta}^{m o d}}$ & $\mathrm{~F}_{H \beta}^{o b s 4}$ & $\log \frac{F_{H \beta}^{o b s} 4}{F_{H \beta}^{m o d}}$ & $\mathrm{~F}_{H \beta}^{o b s 5}$ & $\log \frac{F_{H \beta}^{o b s 5}}{F_{H \beta}^{m o d}}$ \\
\hline 3727. & 204,43 & $-0,25$ & 220,33 & 0,2 & 345,55 & 0,53 & 209,19 & 0,31 & 388,06 & 0,58 \\
\hline 3798. & 45,01 & 0,93 & 73,05 & 1,14 & 42,33 & 0,9 & 53,14 & 0,99 & 46,84 & 0,94 \\
\hline 3820. & 42 & 1,32 & 170,69 & 1,95 & 21,1 & 1,05 & 26,82 & 1,2 & 48,19 & 1,45 \\
\hline 3835. & 28 & 0,58 & 33,18 & 0,66 & 37,77 & 0,71 & 0 & - & 0 & - \\
\hline 3869. & 144,21 & $-0,23$ & 114,3 & $-0,17$ & 131,45 & $-0,1$ & 133,79 & $-0,13$ & 162,14 & $-0,04$ \\
\hline 3889. & 43,63 & 0,62 & 71,19 & 0,84 & 39,48 & 0,58 & 56,73 & 0,73 & 0 & - \\
\hline 3968. & 48,23 & $-0,19$ & 53,69 & 0,03 & 47,87 & $-0,02$ & 65,11 & 0,08 & 94,05 & 0,24 \\
\hline 4026. & 14,32 & 0,59 & 14,95 & 0,62 & 18,12 & 0,71 & 13,93 & 0,65 & 30,98 & 1 \\
\hline 4074 . & 12,49 & 0,18 & 0 & - & 47,96 & 1,3 & 0 & - & 165,41 & 1,84 \\
\hline 4102. & 24,02 & $-0,03$ & 47,15 & 0,27 & 44,75 & 0,24 & 22,13 & $-0,07$ & 31,1 & 0,08 \\
\hline 4267. & 10,12 & 1,16 & 11,1 & 1,2 & 12,65 & 1,26 & 2,38 & 0,6 & 7,78 & 1,11 \\
\hline 4300. & 4,01 & 0,76 & 10,08 & 1,53 & 2,02 & 1 & 0 & - & 10,68 & 1,73 \\
\hline 4341. & 45,48 & 2,18 & 76,46 & 2,28 & 69,7 & 2,24 & 52,3 & 2,12 & 53,28 & 2,13 \\
\hline 4363. & 5,96 & 0,41 & 7,81 & 0,69 & 3,62 & 0,33 & 8,97 & 0,63 & 4,93 & 0,37 \\
\hline 4471. & 1,9 & $-0,62$ & 4,65 & $-0,22$ & 4,96 & $-0,18$ & 5,99 & $-0,05$ & 0 & - \\
\hline 4686. & 1,6 & - & 11,32 & - & 29,38 & 1,04 & 24,14 & 0,04 & 31,69 & 0,16 \\
\hline 4711. & 1,49 & 0,33 & 2,74 & 0,21 & 2,25 & $-0,2$ & 3,59 & $-0,19$ & 3,09 & $-0,25$ \\
\hline 4740 . & 0,89 & 0,25 & 7,28 & 0,75 & 2,83 & 0,02 & 2,48 & $-0,22$ & 2,77 & $-0,17$ \\
\hline 4861. & 100 & 0 & 100 & 0 & 100 & 0 & 100 & 0 & 100 & 0 \\
\hline 4922. & 0 & - & 2,14 & 0,01 & 0 & - & 0,33 & $-0,74$ & 1,04 & $-0,24$ \\
\hline 4959. & 275,86 & 0,08 & 329,96 & 0,19 & 300,14 & 0,13 & 272,99 & 0,09 & 279,15 & 0,1 \\
\hline 5007. & 872,74 & 0,1 & 986,05 & 0,19 & 876,94 & 0,12 & 814,38 & 0,09 & 832,73 & 0,09 \\
\hline 5192. & 11,92 & 1,78 & 0,36 & 0,55 & 0,47 & 0,67 & 0 & - & 0 & - \\
\hline 5199. & 0 & - & 0,35 & $-1,32$ & 0,12 & $-1,63$ & 2,5 & $-0,33$ & 0 & - \\
\hline 5412. & 2,16 & - & 0 & - & 3,63 & 1,26 & 3,71 & 0,42 & 3,8 & 0,43 \\
\hline 5518. & 0,3 & $-0,31$ & 0,73 & 0,26 & 1,76 & 0,77 & 0,44 & 0,17 & 0,39 & 0,11 \\
\hline 5538. & 0,6 & 0,18 & 1,59 & 0,72 & 0 & - & 0,47 & 0,37 & 0 & - \\
\hline 5755. & 6,92 & 0,03 & 0,86 & $-0,45$ & 2,61 & 0,16 & 4,24 & 0,37 & 1,66 & $-0,04$ \\
\hline 5876. & 21,68 & $-0,01$ & 16,98 & $-0,12$ & 16,36 & $-0,12$ & 18,04 & $-0,03$ & 14,35 & $-0,13$ \\
\hline 6300. & 28,22 & 0,14 & 0,13 & $-1,79$ & 1 & $-0,78$ & 12,55 & 0,31 & 1,06 & $-0,76$ \\
\hline 6312. & 4,26 & 0,29 & 0,72 & $-0,29$ & 2,06 & 0,31 & 1,42 & 0,15 & 0,84 & $-0,08$ \\
\hline 6435. & 1,29 & - & 0,28 & - & 0,47 & - & 0,31 & $-0,11$ & 0 & - \\
\hline 6548. & 216,88 & $-0,02$ & 58,2 & $-0,17$ & 42,98 & $-0,17$ & 114,97 & 0,26 & 73,94 & 0,07 \\
\hline 6563. & 284,53 & $-0,02$ & 284,22 & $-0,02$ & 285,15 & $-0,02$ & 284,89 & $-0,02$ & 283,83 & $-0,02$ \\
\hline 6584 . & 644,4 & $-0,01$ & 148,4 & $-0,24$ & 100,56 & $-0,27$ & 310,84 & 0,22 & 195,72 & 0,02 \\
\hline 6678. & 6,29 & 0 & 3,9 & $-0,2$ & 4,3 & $-0,15$ & 5,46 & 0 & 4,43 & $-0,09$ \\
\hline 6716. & 80,11 & 0,11 & 21,47 & $-0,08$ & 16,96 & $-0,04$ & 39,51 & 0,33 & 25,56 & 0,14 \\
\hline 6731. & 64,64 & 0,08 & 16,55 & $-0,13$ & 12,84 & $-0,1$ & 32,7 & 0,31 & 21,17 & 0,12 \\
\hline 7005 . & 0,59 & - & 2,11 & - & 0,48 & - & 0,45 & $-0,25$ & 0,47 & $-0,23$ \\
\hline 7065 . & 3,59 & $-0,14$ & 0 & - & 0,62 & $-0,87$ & 0,77 & $-0,74$ & 1,65 & $-0,41$ \\
\hline
\end{tabular}

Continua na próxima página.. . 
Tabela C.9 - Continuação

\begin{tabular}{|c|c|c|c|c|c|c|c|c|c|c|}
\hline$\lambda(\stackrel{\circ}{A})$ & Janela 1 & $\sigma$ & Janela 2 & $\sigma$ & Janela 3 & $\sigma$ & Janela 4 & $\sigma$ & Janela 5 & $\sigma$ \\
\hline 7135. & 38,73 & $-0,15$ & 24,44 & $-0,18$ & 20,98 & $-0,14$ & 23,03 & $-0,08$ & 21,33 & $-0,11$ \\
\hline 7237 & 7 & - & 2,56 & - & 4,11 & - & 2,12 & - & 3,34 & - \\
\hline 7263 & 1,58 & - & 1,37 & - & 1,23 & - & 1,93 & - & 2,41 & - \\
\hline 7281. & 1,57 & 0,16 & 1,69 & 0,23 & 0,82 & $-0,09$ & 0,34 & $-0,43$ & 1,3 & 0,16 \\
\hline 7325. & 6,95 & 0 & 3,37 & 0,07 & 3 & 0,14 & 4,28 & 0,29 & 6,74 & 0,49 \\
\hline 7532. & 0 & - & 0,92 & 0,66 & 0,74 & 0,57 & 0 & - & 0,72 & 0,55 \\
\hline 7751 & 0 & - & 0 & - & 0 & - & 0 & - & 0 & - \\
\hline
\end{tabular}

Tabela C.10 - Comparação entre fluxos observados e os fluxos previstos pelo modelo de NGC5844 - Fenda Inferior (n $\left.{ }^{\circ} 3\right)$. Janelas 6 a 8.

\begin{tabular}{|c|c|c|c|c|c|c|}
\hline$\lambda(\stackrel{\circ}{A})$ & Janela 6 & $\sigma$ & Janela 7 & $\sigma$ & Janela 8 & $\sigma$ \\
\hline$\lambda$ & $\mathrm{F}_{H \beta}^{o b s 6}$ & $\log \frac{F_{H \beta}^{o b s} 6}{F_{H \beta}^{m o d}}$ & $\mathrm{~F}_{H \beta}^{o b s 7}$ & $\log \frac{F_{H \beta}^{o b s} 7}{F_{H \beta}^{m o d}}$ & $\mathrm{~F}_{H \beta}^{o b s 8}$ & $\log \frac{F_{H \beta}^{o b s} 8}{F_{H \beta}^{m o d}}$ \\
\hline 3727. & 396,49 & 0,59 & 241,79 & 0,24 & 733,02 & 0,31 \\
\hline 3798. & 0 & - & 41,86 & 0,9 & 22,63 & 0,63 \\
\hline 3820. & 156,56 & 1,92 & 55,72 & 1,47 & 30,69 & 1,19 \\
\hline 3835. & 74,45 & 1,01 & 13,97 & 0,28 & 25,17 & 0,54 \\
\hline 3869. & 0 & - & 148,76 & $-0,05$ & 162,95 & $-0,18$ \\
\hline 3889. & 41,76 & 0,6 & 20,97 & 0,31 & 13,46 & 0,11 \\
\hline 3968. & 106,81 & 0,33 & 79,79 & 0,2 & 72,06 & $-0,01$ \\
\hline 4026. & 15,69 & 0,65 & 13,85 & 0,59 & 12,39 & 0,53 \\
\hline 4074. & 14,74 & 0,79 & 10,88 & 0,52 & 10,64 & 0,11 \\
\hline 4102. & 22,7 & $-0,05$ & 42,8 & 0,22 & 30,04 & 0,07 \\
\hline 4267. & 16,48 & 1,37 & 2,97 & 0,63 & 0 & - \\
\hline 4300. & 2,71 & 1,13 & 0 & - & 0 & - \\
\hline 4341. & 59,84 & 2,18 & 60,54 & 2,18 & 46,73 & 2,19 \\
\hline 4363. & 0 & - & 1,31 & $-0,09$ & 0 & - \\
\hline 4471. & 0 & - & 7,66 & 0 & 2,51 & $-0,5$ \\
\hline 4686. & 19,22 & 0,85 & 8,81 & - & 1,13 & - \\
\hline 4711. & 0 & - & 2,81 & 0,22 & 1,31 & 0,27 \\
\hline 4740. & 0,49 & $-0,74$ & 0,42 & $-0,49$ & 0,65 & 0,11 \\
\hline 4861. & 100 & 0 & 100 & 0 & 100 & 0 \\
\hline 4922. & 0 & - & 1,75 & $-0,08$ & 0 & - \\
\hline 4959. & 254,15 & 0,06 & 222,75 & 0,02 & 173,76 & $-0,12$ \\
\hline 5007. & 774,67 & 0,07 & 698,87 & 0,04 & 556,86 & $-0,09$ \\
\hline 5192. & 0 & - & 0 & - & 0 & - \\
\hline 5199. & 0 & - & 0 & - & 0 & - \\
\hline 5412. & 1,25 & 0,8 & 1,18 & - & 1,71 & - \\
\hline 5518. & 0,6 & 0,3 & 0,14 & $-0,45$ & 0,97 & 0,21 \\
\hline
\end{tabular}

Continua na próxima página... 
Tabela C.10 - Continuação

\begin{tabular}{|c|c|c|c|c|c|c|}
\hline$\lambda(\stackrel{\circ}{A})$ & Janela 6 & $\sigma$ & Janela 7 & $\sigma$ & Janela 8 & $\sigma$ \\
\hline 5538. & 1,38 & 0,66 & 0 & - & 0,47 & 0,07 \\
\hline 5755. & 3,82 & 0,33 & 8,05 & 0,53 & 16,79 & 0,42 \\
\hline 5876. & 16,67 & $-0,11$ & 18,95 & $-0,07$ & 21,61 & $-0,02$ \\
\hline 6300. & 19,28 & 0,5 & 31,33 & 0,59 & 65,74 & 0,51 \\
\hline 6312. & 0 & - & 3,06 & 0,34 & 11,51 & 0,72 \\
\hline 6435. & 0,53 & - & 0 & - & 0,14 & - \\
\hline 6548. & 111,5 & 0,25 & 208,11 & 0,38 & 411,7 & 0,26 \\
\hline 6563. & 284,17 & $-0,02$ & 284,37 & $-0,02$ & 284,36 & $-0,02$ \\
\hline 6584. & 317,05 & 0,23 & 618,13 & 0,38 & 1242,03 & 0,27 \\
\hline 6678. & 5,32 & $-0,06$ & 5,07 & $-0,09$ & 6,09 & $-0,01$ \\
\hline 6716. & 43,19 & 0,37 & 72,7 & 0,45 & 153,73 & 0,39 \\
\hline 6731. & 36,23 & 0,36 & 59,05 & 0,43 & 125,21 & 0,36 \\
\hline 7005. & 0 & - & 0,88 & - & 0,49 & - \\
\hline 7065 . & 2,33 & $-0,3$ & 2,44 & $-0,29$ & 3,14 & $-0,19$ \\
\hline 7135. & 20,92 & $-0,15$ & 24,98 & $-0,17$ & 35,32 & $-0,19$ \\
\hline 7237. & 1,84 & - & 1,16 & - & 0,77 & - \\
\hline 7263. & 0 & - & 0,21 & - & 0,44 & - \\
\hline 7281. & 2,33 & 0,37 & 0,19 & $-0,71$ & 0 & - \\
\hline 7325. & 5,71 & 0,41 & 8,82 & 0,48 & 16,92 & 0,39 \\
\hline 7532 . & 0 & - & 0 & - & 0 & - \\
\hline 7751. & 0 & - & 0 & - & 0 & - \\
\hline
\end{tabular}

